\title{
Topics in non-equilibrium quantum statistical mechanics
}

\author{
W. Aschbacher ${ }^{1}$, V. Jakšić ${ }^{2}$, Y. Pautrat ${ }^{3}$, C.-A. Pillet ${ }^{4}$ \\ ${ }^{1}$ Technische Universität München \\ Zentrum Mathematik M5 \\ 85747 Garching, Germany \\ ${ }^{2}$ Department of Mathematics and Statistics \\ McGill University \\ 805 Sherbrooke Street West \\ Montreal, QC, H3A 2K6, Canada \\ ${ }^{3}$ Laboratoire de Mathématiques \\ Université Paris-Sud \\ 91405 Orsay cedex \\ France \\ ${ }^{4}$ CPT-CNRS, UMR 6207 \\ Université du Sud, Toulon-Var, B.P. 20132 \\ F-83957 La Garde Cedex, France
}

June 11, 2005 


\section{Contents}

1 Introduction 3

2 Conceptual framework 3

3 Mathematical framework 5

3.1 Basic concepts ... . . . . . . . . . . . . 5

3.2 Non-equilibrium steady states (NESS) and entropy production . . . . 9

3.3 Structural properties . . . . . . . . . . . . . . . . 10

$3.4 C^{*}$-scattering and NESS .................. 11

4 Open quantum systems 14

4.1 Definition ....................... . . . 14

$4.2 C^{*}$-scattering for open quantum systems . . . . . . . . . . . 16

4.3 The first and second law of thermodynamics . . . . . . . . . . . 17

4.4 Linear response theory . . . . . . . . . . . . . . . . . 18

4.5 Fermi Golden Rule (FGR) thermodynamics . . . . . . . . . . . . 21

5 Free Fermi gas reservoir $\quad \mathbf{2 5}$

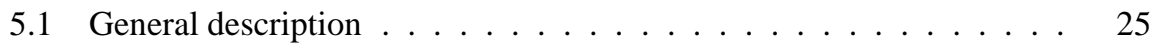

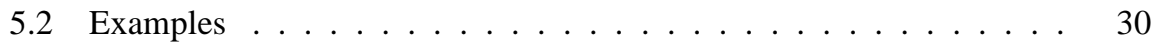

6 The simple electronic black-box (SEBB) model 33

6.1 The model . . . . . . . . . . . . . . . . . 33

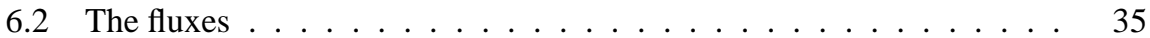

6.3 The equivalent free Fermi gas . . . . . . . . . . . . . . . . 36

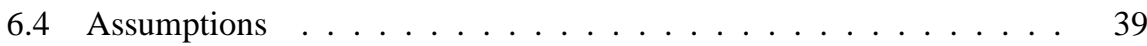

7 Thermodynamics of the SEBB model 42

7.1 Non-equilibrium steady states . . . . . . . . . . . . . . . . . 42

7.2 The Hilbert-Schmidt condition . . . . . . . . . . . . . . . . 43

7.3 The heat and charge fluxes . . . . . . . . . . . . . 44

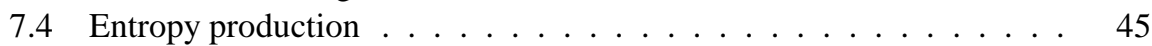

7.5 Equilibrium correlation functions . . . . . . . . . . . . . . 46

7.6 Onsager relations. Kubo formulas. . . . . . . . . . . . . 48

8 FGR thermodynamics of the SEBB model 49

8.1 The weak coupling limit . . . . . . . . . . . . . 49

8.2 Historical digression-Einstein's derivation of the Planck law . . . . 51

8.3 FGR fluxes, entropy production and Kubo formulas . . . . . . . . 53

8.4 From microscopic to FGR thermodynamics . . . . . . . . . . 55

9 Appendix $\quad 56$

9.1 Structural theorems . . . . . . . . . . . . . . . 56

9.2 The Hilbert-Schmidt condition . . . . . . . . . . . . . . 58 


\section{Introduction}

These lecture notes are an expanded version of the lectures given by the second and the fourth author in the summer school "Open Quantum Systems" held in Grenoble, June 16-July 4, 2003. We are grateful to Stéphane Attal and Alain Joye for their hospitality and invitation to speak.

The lecture notes have their root in the recent review article [JP4] and our goal has been to extend and complement certain topics covered in [JP4]. In particular, we will discuss the scattering theory of non-equilibrium steady states (NESS) (this topic has been only quickly reviewed in [JP4]). On the other hand, we will not discuss the spectral theory of NESS which has been covered in detail in [JP4]. Although the lecture notes are self-contained, the reader would benefit from reading them in parallel with [JP4].

Concerning preliminaries, we will assume that the reader is familiar with the material covered in the lecture notes [At, Jo, Pi]. On occasion, we will mention or use some material covered in the lectures [D1, Ja].

As in [JP4], we will work in the mathematical framework of algebraic quantum statistical mechanics. The basic notions of this formalism are reviewed in Section 3. In Section 4 we introduce open quantum systems and describe their basic properties. Linear response theory (this topic has not been discussed in [JP4]) is described in Subsection 4.4. Linear response theory of open quantum systems (Kubo formulas, Onsager relations, Central Limit Theorem) has been studied in the recent papers [FMU, FMSU, AJPP, JPR2].

The second part of the lecture notes (Sections 6-8) is devoted to an example. The model we will discuss is the simplest non-trivial example of the Electronic Black Box Model studied in [AJPP] and we will refer to it as the Simple Electronic Black Box Model (SEBB). The SEBB model is to a large extent exactly solvable-its NESS and entropy production can be exactly computed and Kubo formulas can be verified by an explicit computation. For reasons of space, however, we will not discuss two important topics covered in [AJPP] - the stability theory (which is essentially based on [AM, BM]) and the proof of the Central Limit Theorem. The interested reader may complement Sections 6-8 with the original paper [AJPP] and the recent lecture notes [JKP].

Section 5, in which we discuss statistical mechanics of a free Fermi gas, is the bridge between the two parts of the lecture notes.

Acknowledgment. The research of V.J. was partly supported by NSERC. Part of this work was done while Y.P. was a CRM-ISM postdoc at McGill University and Centre de Recherches Mathématiques in Montreal.

\section{Conceptual framework}

The concept of reference state will play an important role in our discussion of nonequilibrium statistical mechanics. To clarify this notion, let us consider first a classical dynamical system with finitely many degrees of freedom and compact phase space 
$X \subset \mathbb{R}^{n}$. The normalized Lebesgue measure $\mathrm{d} x$ on $X$ provides a physically natural statistics on the phase space in the sense that initial configurations sampled according to it can be considered typical (see [Ru4]). Note that this has nothing to do with the fact that $\mathrm{d} x$ is invariant under the flow of the system-any measure of the form $\rho(x) \mathrm{d} x$ with a strictly positive density $\rho$ would serve the same purpose. The situation is completely different if the system has infinitely many degrees of freedom. In this case, there is no natural replacement for the Lebesgue $\mathrm{d} x$. In fact, a measure on an infinite-dimensional phase space physically describes a thermodynamic state of the system. Suppose for example that the system is Hamiltonian and is in thermal equilibrium at inverse temperature $\beta$ and chemical potential $\mu$. The statistics of such a system is described by the Gibbs measure (grand canonical ensemble). Since two Gibbs measures with different values of the intensive thermodynamic parameters $\beta, \mu$ are mutually singular, initial points sampled according to one of them will be atypical relative to the other. In conclusion, if a system has infinitely many degrees of freedom, we need to specify its initial thermodynamic state by choosing an appropriate reference measure. As in the finite-dimensional case, this measure may not be invariant under the flow. It also may not be uniquely determined by the physical situation we wish to describe.

The situation in quantum mechanics is very similar. The Schrödinger representation of a system with finitely many degrees of freedom is (essentially) uniquely determined and the natural statistics is provided by any strictly positive density matrix on the Hilbert space of the system. For systems with infinitely many degrees of freedom there is no such natural choice. The consequences of this fact are however more drastic than in the classical case. There is no natural choice of a Hilbert space in which the system can be represented. To induce a representation, we must specify the thermodynamic state of the system by choosing an appropriate reference state. The algebraic formulation of quantum statistical mechanics provides a mathematical framework to study such infinite system in a representation independent way.

One may object that no real physical system has an infinite number of degrees of freedom and that, therefore, a unique natural reference state always exists. There are however serious methodological reasons to consider this mathematical idealization. Already in equilibrium statistical mechanics the fundamental phenomena of phase transition can only be characterized in a mathematically precise way within such an idealization: A quantum system with finitely many degrees of freedom has a unique thermal equilibrium state. Out of equilibrium, relaxation towards a stationary state and emergence of steady currents can not be expected from the quasi-periodic time evolution of a finite system.

In classical non-equilibrium statistical mechanics there exists an alternative approach to this idealization. A system forced by a non-Hamiltonian or time-dependent force can be driven towards a non-equilibrium steady state, provided the energy supplied by the external source is removed by some thermostat. This micro-canonical point of view has a number of advantages over the canonical, infinite system idealization. A dynamical system with a relatively small number of degrees of freedom can easily be explored on a computer (numerical integration, iteration of Poincaré sections, ...). A large body of "experimental facts" is currently available from the results of such investigations (see [EM, Do] for an introduction to the techniques and a lucid exposition of the results). From a more theoretical perspective, the full machinery of 
finite-dimensional dynamical system theory becomes available in the micro-canonical approach. The Chaotic Hypothesis introduced in [CG1, CG2] is an attempt to exploit this fact. It justifies phenomenological thermodynamics (Onsager relations, linear response theory, fluctuation-dissipation formulas,...) and has lead to more unexpected results like the Gallavotti-Cohen Fluctuation Theorem. The major drawback of the micro-canonical point of view is the non-Hamiltonian nature of the dynamics, which makes it inappropriate to quantum-mechanical treatment.

The two approaches described above are not completely unrelated. For example, we shall see that the signature of a non-equilibrium steady state in quantum mechanics is its singularity with respect to the reference state, a fact which is well understood in the classical, micro-canonical approach (see Chapter 10 of [EM]). More speculatively, one can expect a general equivalence principle for dynamical (micro-canonical and canonical) ensembles (see [Ru5]). The results in this direction are quite scarce and much work remains to be done.

\section{Mathematical framework}

In this section we describe the mathematical formalism of algebraic quantum statistical mechanics. Our presentation follows [JP4] and is suited for applications to nonequilibrium statistical mechanics. Most of the material in this section is well known and the proofs can be found, for example, in [BR1, BR2, DJP, Ha, OP, Ta]. The proofs of the results described in Subsection 3.3 are given in Appendix 9.1.

\subsection{Basic concepts}

The starting point of our discussion is a pair $(\mathcal{O}, \tau)$, where $\mathcal{O}$ is a $C^{*}$-algebra with a unit $I$ and $\tau$ is a $C^{*}$-dynamics (a strongly continuous group $\mathbb{R} \ni t \mapsto \tau^{t}$ of $*$ automorphisms of $\mathcal{O}$ ). The elements of $\mathcal{O}$ describe physical observables of the quantum system under consideration and the group $\tau$ specifies their time evolution. The pair $(\mathcal{O}, \tau)$ is sometimes called a $C^{*}$-dynamical system.

In the sequel, by the strong topology on $\mathcal{O}$ we will always mean the usual norm topology of $\mathcal{O}$ as Banach space. The $C^{*}$-algebra of all bounded operators on a Hilbert space $\mathcal{H}$ is denoted by $\mathcal{B}(\mathcal{H})$.

A state $\omega$ on the $C^{*}$-algebra $\mathcal{O}$ is a normalized $(\omega(I)=1)$, positive $\left(\omega\left(A^{*} A\right) \geq 0\right)$, linear functional on $\mathcal{O}$. It specifies a possible physical state of the quantum mechanical system. If the system is in the state $\omega$ at time zero, the quantum mechanical expectation value of the observable $A$ at time $t$ is given by $\omega\left(\tau^{t}(A)\right)$. Thus, states evolve in the Schrödinger picture according to $\omega_{t}=\omega \circ \tau^{t}$. The set $E(\mathcal{O})$ of all states on $\mathcal{O}$ is a convex, weak-* compact subset of the Banach space dual $\mathcal{O}^{*}$ of $\mathcal{O}$.

A linear functional $\eta \in \mathcal{O}^{*}$ is called $\tau$-invariant if $\eta \circ \tau^{t}=\eta$ for all $t$. The set of all $\tau$-invariant states is denoted by $E(\mathcal{O}, \tau)$. This set is always non-empty. A state $\omega \in E(\mathcal{O}, \tau)$ is called ergodic if

$$
\lim _{T \rightarrow \infty} \frac{1}{2 T} \int_{-T}^{T} \omega\left(B^{*} \tau^{t}(A) B\right) \mathrm{d} t=\omega(A) \omega\left(B^{*} B\right),
$$


and mixing if

$$
\lim _{|t| \rightarrow \infty} \omega\left(B^{*} \tau^{t}(A) B\right)=\omega(A) \omega\left(B^{*} B\right)
$$

for all $A, B \in \mathcal{O}$.

Let $\left(\mathcal{H}_{\eta}, \pi_{\eta}, \Omega_{\eta}\right)$ be the GNS representation associated to a positive linear functional $\eta \in \mathcal{O}^{*}$. The enveloping von Neumann algebra of $\mathcal{O}$ associated to $\eta$ is $\mathfrak{M}_{\eta} \equiv$ $\pi_{\eta}(\mathcal{O})^{\prime \prime} \subset \mathcal{B}\left(\mathcal{H}_{\eta}\right)$. A linear functional $\mu \in \mathcal{O}^{*}$ is normal relative to $\eta$ or $\eta$-normal, denoted $\mu \ll \eta$, if there exists a trace class operator $\rho_{\mu}$ on $\mathcal{H}_{\eta}$ such that $\mu(\cdot)=$ $\operatorname{Tr}\left(\rho_{\mu} \pi_{\eta}(\cdot)\right)$. Any $\eta$-normal linear functional $\mu$ has a unique normal extension to $\mathfrak{M}_{\eta}$. We denote by $\mathcal{N}_{\eta}$ the set of all $\eta$-normal states. $\mu \ll \eta$ iff $\mathcal{N}_{\mu} \subset \mathcal{N}_{\eta}$.

A state $\omega$ is ergodic iff, for all $\mu \in \mathcal{N}_{\omega}$ and $A \in \mathcal{O}$,

$$
\lim _{T \rightarrow \infty} \frac{1}{2 T} \int_{-T}^{T} \mu\left(\tau^{t}(A)\right) \mathrm{d} t=\omega(A) .
$$

For this reason ergodicity is sometimes called return to equilibrium in mean; see [Ro1, Ro2]. Similarly, $\omega$ is mixing (or returns to equilibrium) iff

$$
\lim _{|t| \rightarrow \infty} \mu\left(\tau^{t}(A)\right)=\omega(A)
$$

for all $\mu \in \mathcal{N}_{\omega}$ and $A \in \mathcal{O}$.

Let $\eta$ and $\mu$ be two positive linear functionals in $\mathcal{O}^{*}$, and suppose that $\eta \geq \phi \geq 0$ for some $\mu$-normal $\phi$ implies $\phi=0$. We then say that $\eta$ and $\mu$ are mutually singular (or orthogonal), and write $\eta \perp \mu$. An equivalent (more symmetric) definition is: $\eta \perp \mu$ iff $\eta \geq \phi \geq 0$ and $\mu \geq \phi \geq 0$ imply $\phi=0$.

Two positive linear functionals $\eta$ and $\mu$ in $\mathcal{O}^{*}$ are called disjoint if $\mathcal{N}_{\eta} \cap \mathcal{N}_{\mu}=\emptyset$. If $\eta$ and $\mu$ are disjoint, then $\eta \perp \mu$. The converse does not hold-it is possible that $\eta$ and $\mu$ are mutually singular but not disjoint.

To elucidate further these important notions, we recall the following well-known results; see Lemmas 4.1.19 and 4.2.8 in [BR1].

Proposition 3.1 Let $\mu_{1}, \mu_{2} \in \mathcal{O}^{*}$ be two positive linear functionals and $\mu=\mu_{1}+\mu_{2}$. Then the following statements are equivalent:

(i) $\mu_{1} \perp \mu_{2}$.

(ii) There exists a projection $P$ in $\pi_{\mu}(\mathcal{O})^{\prime}$ such that

$$
\mu_{1}(A)=\left(P \Omega_{\mu}, \pi_{\mu}(A) \Omega_{\mu}\right), \quad \mu_{2}(A)=\left((I-P) \Omega_{\mu}, \pi_{\mu}(A) \Omega_{\mu}\right) .
$$

(iii) The GNS representation $\left(\mathcal{H}_{\mu}, \pi_{\mu}, \Omega_{\mu}\right)$ is a direct sum of the two GNS representations $\left(\mathcal{H}_{\mu_{1}}, \pi_{\mu_{1}}, \Omega_{\mu_{1}}\right)$ and $\left(\mathcal{H}_{\mu_{2}}, \pi_{\mu_{2}}, \Omega_{\mu_{2}}\right)$, i.e.,

$$
\mathcal{H}_{\mu}=\mathcal{H}_{\mu_{1}} \oplus \mathcal{H}_{\mu_{2}}, \quad \pi_{\mu}=\pi_{\mu_{1}} \oplus \pi_{\mu_{2}}, \quad \Omega_{\mu}=\Omega_{\mu_{1}} \oplus \Omega_{\mu_{2}} .
$$

Proposition 3.2 Let $\mu_{1}, \mu_{2} \in \mathcal{O}^{*}$ be two positive linear functionals and $\mu=\mu_{1}+\mu_{2}$. Then the following statements are equivalent: 
(i) $\mu_{1}$ and $\mu_{2}$ are disjoint.

(ii) There exists a projection $P$ in $\pi_{\mu}(\mathcal{O})^{\prime} \cap \pi_{\mu}(\mathcal{O})^{\prime \prime}$ such that

$$
\mu_{1}(A)=\left(P \Omega_{\mu}, \pi_{\mu}(A) \Omega_{\mu}\right), \quad \mu_{2}(A)=\left((I-P) \Omega_{\mu}, \pi_{\mu}(A) \Omega_{\mu}\right) .
$$

Let $\eta, \mu \in \mathcal{O}^{*}$ be two positive linear functionals. The functional $\eta$ has a unique decomposition $\eta=\eta_{n}+\eta_{s}$, where $\eta_{n}, \eta_{s}$ are positive, $\eta_{n} \ll \mu$, and $\eta_{s} \perp \mu$. The uniqueness of the decomposition implies that if $\eta$ is $\tau$-invariant, then so are $\eta_{n}$ and $\eta_{s}$.

To elucidate the nature of this decomposition we need to recall the notions of the universal representation and the universal enveloping von Neumann algebra of $\mathcal{O}$; see Section III.2 in [Ta] and Section 10.1 in [KR].

Set

$$
\mathcal{H}_{\mathrm{un}} \equiv \bigoplus_{\omega \in E(\mathcal{O})} \mathcal{H}_{\omega}, \quad \pi_{\mathrm{un}} \equiv \bigoplus_{\omega \in E(\mathcal{O})} \pi_{\omega}, \quad \mathfrak{M}_{\mathrm{un}} \equiv \pi_{\mathrm{un}}(\mathcal{O})^{\prime \prime}
$$

$\left(\mathcal{H}_{\mathrm{un}}, \pi_{\mathrm{un}}\right)$ is a faithful representation. It is called the universal representation of $\mathcal{O}$. $\mathfrak{M}_{\text {un }} \subset \mathcal{B}\left(\mathcal{H}_{\text {un }}\right)$ is its universal enveloping von Neumann algebra. For any $\omega \in E(\mathcal{O})$ the map

$$
\begin{aligned}
& \pi_{\text {un }}(\mathcal{O}) \rightarrow \pi_{\omega}(\mathcal{O}) \\
& \pi_{\text {un }}(A) \mapsto \pi_{\omega}(A),
\end{aligned}
$$

extends to a surjective $*$-morphism $\tilde{\pi}_{\omega}: \mathfrak{M}_{\mathrm{un}} \rightarrow \mathfrak{M}_{\omega}$. It follows that $\omega$ uniquely extends to a normal state $\tilde{\omega}(\cdot) \equiv\left(\Omega_{\omega}, \tilde{\pi}_{\omega}(\cdot) \Omega_{\omega}\right)$ on $\mathfrak{M}_{\text {un }}$. Moreover, one easily shows that

$$
\operatorname{Ker} \tilde{\pi}_{\omega}=\left\{A \in \mathfrak{M}_{\mathrm{un}} \mid \tilde{\nu}(A)=0 \text { for any } \nu \in \mathcal{N}_{\omega}\right\} .
$$

Since Ker $\tilde{\pi}_{\omega}$ is a $\sigma$-weakly closed two sided ideal in $\mathfrak{M}_{\text {un }}$, there exists an orthogonal projection $p_{\omega} \in \mathfrak{M}_{\text {un }} \cap \mathfrak{M}_{\text {un }}^{\prime}$ such that Ker $\tilde{\pi}_{\omega}=p_{\omega} \mathfrak{M}_{\text {un }}$. The orthogonal projection $z_{\omega} \equiv I-p_{\omega} \in \mathfrak{M}_{\text {un }} \cap \mathfrak{M}_{\text {un }}^{\prime}$ is called the support projection of the state $\omega$. The restriction of $\tilde{\pi}_{\omega}$ to $z_{\omega} \mathfrak{M}_{\mathrm{un}}$ is an isomorphism between the von Neumann algebras $z_{\omega} \mathfrak{M}_{\text {un }}$ and $\mathfrak{M}_{\omega}$. We shall denote by $\phi_{\omega}$ the inverse isomorphism.

Let now $\eta, \mu \in \mathcal{O}^{*}$ be two positive linear functionals. By scaling, without loss of generality we may assume that they are states. Since $\tilde{\eta}$ is a normal state on $\mathfrak{M}_{\text {un }}$ it follows that $\tilde{\eta} \circ \phi_{\mu}$ is a normal state on $\mathfrak{M}_{\mu}$ and hence that $\eta_{n} \equiv \tilde{\eta} \circ \phi_{\mu} \circ \pi_{\mu}$ defines a $\mu$-normal positive linear functional on $\mathcal{O}$. Moreover, from the relation $\phi_{\mu} \circ \pi_{\mu}(A)=$ $z_{\mu} \pi_{\mathrm{un}}(A)$ it follows that

$$
\eta_{n}(A)=\left(\Omega_{\eta}, \tilde{\pi}_{\eta}\left(z_{\mu}\right) \pi_{\eta}(A) \Omega_{\eta}\right) .
$$

Setting

$$
\eta_{s}(A) \equiv\left(\Omega_{\eta}, \tilde{\pi}_{\eta}\left(p_{\mu}\right) \pi_{\eta}(A) \Omega_{\eta}\right),
$$

we obtain a decomposition $\eta=\eta_{n}+\eta_{s}$. To show that $\eta_{s} \perp \mu$ let $\omega$ be a $\mu$-normal positive linear functional on $\mathcal{O}$ such that $\eta_{s} \geq \omega$. By the unicity of the normal extension $\tilde{\eta}_{s}$ one has $\tilde{\eta}_{s}(A)=\tilde{\eta}\left(p_{\mu} A\right)$ for $A \in \mathfrak{M}_{\text {un }}$. Since $\pi_{\text {un }}(\mathcal{O})$ is $\sigma$-strongly dense in 
$\mathfrak{M}_{\text {un }}$ it follows from the inequality $\tilde{\eta}_{s} \circ \pi_{\text {un }} \geq \tilde{\omega} \circ \pi_{\text {un }}$ that $\tilde{\eta}\left(p_{\mu} A\right) \geq \tilde{\omega}(A)$ for any positive $A \in \mathfrak{M}_{\text {un }}$. Since $\omega$ is $\mu$-normal, it further follows from Equ. (3.1) that $\omega(A)=\tilde{\omega}\left(\pi_{\mathrm{un}}(A)\right)=\tilde{\omega}\left(z_{\mu} \pi_{\mathrm{un}}(A)\right) \leq \tilde{\eta}\left(p_{\mu} z_{\mu} \pi_{\mathrm{un}}(A)\right)=0$ for any positive $A \in \mathcal{O}$, i.e., $\omega=0$. Since $\tilde{\pi}_{\eta}$ is surjective, one has $\tilde{\pi}_{\eta}\left(z_{\mu}\right) \in \mathfrak{M}_{\eta} \cap \mathfrak{M}_{\eta}^{\prime}$ and, by Proposition 3.2, the functionals $\eta_{n}$ and $\eta_{s}$ are disjoint.

Two states $\omega_{1}$ and $\omega_{2}$ are called quasi-equivalent if $\mathcal{N}_{\omega_{1}}=\mathcal{N}_{\omega_{2}}$. They are called unitarily equivalent if their GNS representations $\left(\mathcal{H}_{\omega_{j}}, \pi_{\omega_{j}}, \Omega_{\omega_{j}}\right)$ are unitarily equivalent, namely if there is a unitary $U: \mathcal{H}_{\omega_{1}} \rightarrow \mathcal{H}_{\omega_{2}}$ such that $U \Omega_{\omega_{1}}=\Omega_{\omega_{2}}$ and $U \pi_{\omega_{1}}(\cdot)=\pi_{\omega_{2}}(\cdot) U$. Clearly, unitarily equivalent states are quasi-equivalent.

If $\omega$ is $\tau$-invariant, then there exists a unique self-adjoint operator $L$ on $\mathcal{H}_{\omega}$ such that

$$
L \Omega_{\omega}=0, \quad \pi_{\omega}\left(\tau^{t}(A)\right)=\mathrm{e}^{\mathrm{i} t L} \pi_{\omega}(A) \mathrm{e}^{-\mathrm{i} t L} .
$$

We will call $L$ the $\omega$-Liouvillean of $\tau$.

The state $\omega$ is called factor state (or primary state) if its enveloping von Neumann algebra $\mathfrak{M}_{\omega}$ is a factor, namely if $\mathfrak{M}_{\omega} \cap \mathfrak{M}_{\omega}^{\prime}=\mathbb{C} I$. By Proposition $3.2 \omega$ is a factor state iff it cannot be written as a nontrivial convex combination of disjoint states. This implies that if $\omega$ is a factor state and $\mu$ is a positive linear functional in $\mathcal{O}^{*}$, then either $\omega \ll \mu$ or $\omega \perp \mu$.

Two factor states $\omega_{1}$ and $\omega_{2}$ are either quasi-equivalent or disjoint. They are quasiequivalent iff $\left(\omega_{1}+\omega_{2}\right) / 2$ is also a factor state (this follows from Theorem 4.3.19 in [BR1]).

The state $\omega$ is called modular if there exists a $C^{*}$-dynamics $\sigma_{\omega}$ on $\mathcal{O}$ such that $\omega$ is a $\left(\sigma_{\omega},-1\right)$-KMS state. If $\omega$ is modular, then $\Omega_{\omega}$ is a separating vector for $\mathfrak{M}_{\omega}$, and we denote by $\Delta_{\omega}, J$ and $\mathcal{P}$ the modular operator, the modular conjugation and the natural cone associated to $\Omega_{\omega}$. To any $C^{*}$-dynamics $\tau$ on $\mathcal{O}$ one can associate a unique self-adjoint operator $L$ on $\mathcal{H}_{\omega}$ such that for all $t$

$$
\pi_{\omega}\left(\tau^{t}(A)\right)=\mathrm{e}^{\mathrm{i} t L} \pi_{\omega}(A) \mathrm{e}^{-\mathrm{i} t L}, \quad \mathrm{e}^{-\mathrm{i} t L} \mathcal{P}=\mathcal{P} .
$$

The operator $L$ is called standard Liouvillean of $\tau$ associated to $\omega$. If $\omega$ is $\tau$-invariant, then $L \Omega_{\omega}=0$, and the standard Liouvillean is equal to the $\omega$-Liouvillean of $\tau$.

The importance of the standard Liouvillean $L$ stems from the fact that if a state $\eta$ is $\omega$-normal and $\tau$-invariant, then there exists a unique vector $\Omega_{\eta} \in \operatorname{Ker} L \cap \mathcal{P}$ such that $\eta(\cdot)=\left(\Omega_{\eta}, \pi_{\omega}(\cdot) \Omega_{\eta}\right)$. This fact has two important consequences. On one hand, if $\eta$ is $\omega$-normal and $\tau$-invariant, then some ergodic properties of the quantum dynamical system $(\mathcal{O}, \tau, \eta)$ can be described in terms of the spectral properties of $L$; see [JP2, Pi]. On the other hand, if $\operatorname{Ker} L=\{0\}$, then the $C^{*}$-dynamics $\tau$ has no $\omega$-normal invariant states. The papers [BFS, DJ, FM1, FM2, FMS, JP1, JP2, JP3, Me1, Me2, Og] are centered around this set of ideas.

In quantum statistical mechanics one also encounters $L^{p}$-Liouvilleans, for $p \in$ $[1, \infty]$ (the standard Liouvillean is equal to the $L^{2}$-Liouvillean). The $L^{p}$-Liouvilleans are closely related to the Araki-Masuda $L^{p}$-spaces [ArM]. $L^{1}$ and $L^{\infty}$-Liouvilleans have played a central role in the spectral theory of NESS developed in [JP5]. The use of other $L^{p}$-Liouvilleans is more recent (see [JPR2]) and they will not be discussed in this lecture. 


\subsection{Non-equilibrium steady states (NESS) and entropy production}

The central notions of non-equilibrium statistical mechanics are non-equilibrium steady states (NESS) and entropy production. Our definition of NESS follows closely the idea of Ruelle that a "natural" steady state should provide the statistics, over large time intervals $[0, t]$, of initial configurations of the system which are typical with respect to the reference state [Ru3]. The definition of entropy production is more problematic since there is no physically satisfactory definition of the entropy itself out of equilibrium; see [Ga1, Ru2, Ru5, Ru7] for a discussion. Our definition of entropy production is motivated by classical dynamics where the rate of change of thermodynamic (Clausius) entropy can sometimes be related to the phase space contraction rate [Ga2, RC]. The latter is related to the Gibbs entropy (as shown for example in [Ru3]) which is nothing else but the relative entropy with respect to the natural reference state; see [JPR1] for a detailed discussion in a more general context. Thus, it seems reasonable to define the entropy production as the rate of change of the relative entropy with respect to the reference state $\omega$.

Let $(\mathcal{O}, \tau)$ be a $C^{*}$-dynamical system and $\omega$ a given reference state. The NESS associated to $\omega$ and $\tau$ are the weak-* limit points of the time averages along the trajectory $\omega \circ \tau^{t}$. In other words, if

$$
\langle\omega\rangle_{t} \equiv \frac{1}{t} \int_{0}^{t} \omega \circ \tau^{s} \mathrm{~d} s,
$$

then $\omega_{+}$is a NESS associated to $\omega$ and $\tau$ if there exists a net $t_{\alpha} \rightarrow \infty$ such that $\langle\omega\rangle_{t_{\alpha}}(A) \rightarrow \omega_{+}(A)$ for all $A \in \mathcal{O}$. We denote by $\Sigma_{+}(\omega, \tau)$ the set of such NESS. One easily sees that $\Sigma_{+}(\omega, \tau) \subset E(\mathcal{O}, \tau)$. Moreover, since $E(\mathcal{O})$ is weak-* compact, $\Sigma_{+}(\omega, \tau)$ is non-empty.

As already mentioned, our definition of entropy production is based on the concept of relative entropy. The relative entropy of two density matrices $\rho$ and $\omega$ is defined, by analogy with the relative entropy of two measures, by the formula

$$
\operatorname{Ent}(\rho \mid \omega) \equiv \operatorname{Tr}(\rho(\log \omega-\log \rho))
$$

It is easy to show that $\operatorname{Ent}(\rho \mid \omega) \leq 0$. Let $\varphi_{i}$ an orthonormal eigenbasis of $\rho$ and by $p_{i}$ the corresponding eigenvalues. Then $p_{i} \in[0,1]$ and $\sum_{i} p_{i}=1$. Let $q_{i} \equiv\left(\varphi_{i}, \omega \varphi_{i}\right)$. Clearly, $q_{i} \in[0,1]$ and $\sum_{i} q_{i}=\operatorname{Tr} \omega=1$. Applying Jensen's inequality twice we derive

$$
\begin{aligned}
\operatorname{Ent}(\rho \mid \omega) & =\sum_{i} p_{i}\left(\left(\varphi_{i}, \log \omega \varphi_{i}\right)-\log p_{i}\right) \\
& \leq \sum_{i} p_{i}\left(\log q_{i}-\log p_{i}\right) \leq \log \sum_{i} q_{i}=0 .
\end{aligned}
$$

Hence $\operatorname{Ent}(\rho \mid \omega) \leq 0$. It is also not difficult to show that $\operatorname{Ent}(\rho \mid \omega)=0$ iff $\rho=\omega$; see $[\mathrm{OP}]$. Using the concept of relative modular operators, Araki has extended the notion of relative entropy to two arbitrary states on a $C^{*}$-algebra [Ar1, Ar2]. We refer the reader to $[\mathrm{Ar} 1, \mathrm{Ar} 2, \mathrm{DJP}, \mathrm{OP}]$ for the definition of the Araki relative entropy and its basic properties. Of particular interest to us is that $\operatorname{Ent}(\rho \mid \omega) \leq 0$ still holds, with equality if and only if $\rho=\omega$. 
In these lecture notes we will define entropy production only in a perturbative context (for a more general approach see [JPR2]). Denote by $\delta$ the generator of the group $\tau$ i.e., $\tau^{t}=\mathrm{e}^{t \delta}$, and assume that the reference state $\omega$ is invariant under $\tau$. For $V=V^{*} \in \mathcal{O}$ we set $\delta_{V} \equiv \delta+\mathrm{i}[V, \cdot]$ and denote by $\tau_{V}^{t} \equiv \mathrm{e}^{t \delta_{V}}$ the corresponding perturbed $C^{*}$-dynamics (such perturbations are often called local, see [Pi]). Starting with a state $\rho \in \mathcal{N}_{\omega}$, the entropy is pumped out of the system by the perturbation $V$ at a mean rate

$$
-\frac{1}{t}\left(\operatorname{Ent}\left(\rho \circ \tau_{V}^{t} \mid \omega\right)-\operatorname{Ent}(\rho \mid \omega)\right)
$$

Suppose that $\omega$ is a modular state for a $C^{*}$-dynamics $\sigma_{\omega}^{t}$ and denote by $\delta_{\omega}$ the generator of $\sigma_{\omega}$. If $V \in \operatorname{Dom}\left(\delta_{\omega}\right)$, then one can prove the following entropy balance equation

$$
\operatorname{Ent}\left(\rho \circ \tau_{V}^{t} \mid \omega\right)=\operatorname{Ent}(\rho \mid \omega)-\int_{0}^{t} \rho\left(\tau_{V}^{s}\left(\sigma_{V}\right)\right) \mathrm{d} s
$$

where

$$
\sigma_{V} \equiv \delta_{\omega}(V)
$$

is the entropy production observable (see [JP6, JP7]). In quantum mechanics $\sigma_{V}$ plays the role of the phase space contraction rate of classical dynamical systems (see [JPR1]). We define the entropy production rate of a NESS

$$
\rho_{+}=\mathrm{w}^{*}-\lim \frac{1}{t_{\alpha}} \int_{0}^{t_{\alpha}} \rho \circ \tau_{V}^{s} \mathrm{~d} s \in \Sigma_{+}\left(\rho, \tau_{V}\right),
$$

by

$$
\operatorname{Ep}\left(\rho_{+}\right) \equiv-\lim _{\alpha} \frac{1}{t_{\alpha}}\left(\operatorname{Ent}\left(\rho \circ \tau_{V}^{t_{\alpha}} \mid \omega\right)-\operatorname{Ent}(\rho \mid \omega)\right)=\rho_{+}\left(\sigma_{V}\right) .
$$

Since $\operatorname{Ent}\left(\rho \circ \tau_{V}^{t} \mid \omega\right) \leq 0$, an immediate consequence of this equation is that, for $\rho_{+} \in \Sigma_{+}\left(\rho, \tau_{V}\right)$

$$
\operatorname{Ep}\left(\rho_{+}\right) \geq 0
$$

We emphasize that the observable $\sigma_{V}$ depends both on the reference state $\omega$ and on the perturbation $V$. As we shall see in the next section, $\sigma_{V}$ is related to the thermodynamic fluxes across the system produced by the perturbation $V$ and the positivity of entropy production is the statement of the second law of thermodynamics.

\subsection{Structural properties}

In this subsection we shall discuss structural properties of NESS and entropy production following [JP4]. The proofs are given in Appendix 9.1.

First, we will discuss the dependence of $\Sigma_{+}\left(\omega, \tau_{V}\right)$ on the reference state $\omega$. On physical grounds, one may expect that if $\omega$ is sufficiently regular and $\eta$ is $\omega$-normal, then $\Sigma_{+}\left(\eta, \tau_{V}\right)=\Sigma_{+}\left(\omega, \tau_{V}\right)$.

Theorem 3.3 Assume that $\omega$ is a factor state on the $C^{*}$-algebra $\mathcal{O}$ and that, for all $\eta \in \mathcal{N}_{\omega}$ and $A, B \in \mathcal{O}$,

$$
\lim _{T \rightarrow \infty} \frac{1}{T} \int_{0}^{T} \eta\left(\left[\tau_{V}^{t}(A), B\right]\right) \mathrm{d} t=0
$$


holds (weak asymptotic abelianness in mean). Then $\Sigma_{+}\left(\eta, \tau_{V}\right)=\Sigma_{+}\left(\omega, \tau_{V}\right)$ for all $\eta \in \mathcal{N}_{\omega}$.

The second structural property we would like to mention is:

Theorem 3.4 Let $\eta \in \mathcal{O}^{*}$ be $\omega$-normal and $\tau_{V}$-invariant. Then $\eta\left(\sigma_{V}\right)=0$. In particular, the entropy production of the normal part of any NESS is equal to zero.

If $\operatorname{Ent}(\eta \mid \omega)>-\infty$, then Theorem 3.4 is an immediate consequence of the entropy balance equation (3.3). The case $\operatorname{Ent}(\eta \mid \omega)=-\infty$ has been treated in [JP7] and the proof requires the full machinery of Araki's perturbation theory. We will not reproduce it here.

If $\omega_{+}$is a factor state, then either $\omega_{+} \ll \omega$ or $\omega_{+} \perp \omega$. Hence, Theorem 3.4 yields:

Corollary 3.5 If $\omega_{+}$is a factor state and $\operatorname{Ep}\left(\omega_{+}\right)>0$, then $\omega_{+} \perp \omega$. If $\omega$ is also a factor state, then $\omega_{+}$and $\omega$ are disjoint.

Certain structural properties can be characterized in terms of the standard Liouvillean. Let $L$ be the standard Liouvillean associated to $\tau$ and $L_{V}$ the standard Liouvillean associated to $\tau_{V}$. By the well-known Araki's perturbation formula, one has $L_{V}=L+V-J V J$ (see [DJP, Pi]).

Theorem 3.6 Assume that $\omega$ is modular.

(i) Under the assumptions of Theorem 3.3, if $\operatorname{Ker} L_{V} \neq\{0\}$, then it is one-dimensional and there exists a unique normal, $\tau_{V}$-invariant state $\omega_{V}$ such that

$$
\Sigma_{+}\left(\omega, \tau_{V}\right)=\left\{\omega_{V}\right\}
$$

(ii) If $\operatorname{Ker} L_{V}=\{0\}$, then any NESS in $\Sigma_{+}\left(\omega, \tau_{V}\right)$ is purely singular.

(iii) If Ker $L_{V}$ contains a separating vector for $\mathfrak{M}_{\omega}$, then $\Sigma_{+}\left(\omega, \tau_{V}\right)$ contains a unique state $\omega_{+}$and this state is $\omega$-normal.

\section{4 $C^{*}$-scattering and NESS}

Let $(\mathcal{O}, \tau)$ be a $C^{*}$-dynamical system and $V$ a local perturbation. The abstract $C^{*}$ scattering approach to the study of NESS is based on the following assumption:

Assumption (S) The strong limit

$$
\alpha_{V}^{+} \equiv \mathrm{s}-\lim _{t \rightarrow \infty} \tau^{-t} \circ \tau_{V}^{t}
$$

exists.

The map $\alpha_{V}^{+}$is an isometric *-endomorphism of $\mathcal{O}$, and is often called Møller morphism. $\alpha_{V}^{+}$is one-to-one but it is generally not onto, namely

$$
\mathcal{O}_{+} \equiv \operatorname{Ran} \alpha_{V}^{+} \neq \mathcal{O}
$$


Since $\alpha_{V}^{+} \circ \tau_{V}^{t}=\tau^{t} \circ \alpha_{V}^{+}$, the pair $\left(\mathcal{O}_{+}, \tau\right)$ is a $C^{*}$-dynamical system and $\alpha_{V}^{+}$is an isomorphism between the dynamical systems $\left(\mathcal{O}, \tau_{V}\right)$ and $\left(\mathcal{O}_{+}, \tau\right)$.

If the reference state $\omega$ is $\tau$-invariant, then $\omega_{+}=\omega \circ \alpha_{V}^{+}$is the unique NESS associated to $\omega$ and $\tau_{V}$ and

$$
\mathrm{w}_{t \rightarrow \infty}^{*}-\lim _{t \rightarrow \infty} \omega \circ \tau_{V}^{t}=\omega_{+} .
$$

Note in particular that if $\omega$ is a $(\tau, \beta)$-KMS state, then $\omega_{+}$is a $\left(\tau_{V}, \beta\right)$-KMS state.

The map $\alpha_{V}^{+}$is the algebraic analog of the wave operator in Hilbert space scattering theory. A simple and useful result in Hilbert space scattering theory is the Cook criterion for the existence of the wave operator. Its algebraic analog is:

Proposition 3.7 (i) Assume that there exists a dense subset $\mathcal{O}_{0} \subset \mathcal{O}$ such that for all $A \in \mathcal{O}_{0}$,

$$
\int_{0}^{\infty}\left\|\left[V, \tau_{V}^{t}(A)\right]\right\| \mathrm{d} t<\infty .
$$

Then Assumption (S) holds.

(ii) Assume that there exists a dense subset $\mathcal{O}_{1} \subset \mathcal{O}$ such that for all $A \in \mathcal{O}_{1}$,

$$
\int_{0}^{\infty}\left\|\left[V, \tau^{t}(A)\right]\right\| \mathrm{d} t<\infty .
$$

Then $\mathcal{O}_{+}=\mathcal{O}$ and $\alpha_{V}^{+}$is a $*$-automorphism of $\mathcal{O}$.

Proof. For all $A \in \mathcal{O}$ we have

$$
\begin{aligned}
& \tau^{-t_{2}} \circ \tau_{V}^{t_{2}}(A)-\tau^{-t_{1}} \circ \tau_{V}^{t_{1}}(A)=\mathrm{i} \int_{t_{1}}^{t_{2}} \tau^{-t}\left(\left[V, \tau_{V}^{t}(A)\right]\right) \mathrm{d} t, \\
& \tau_{V}^{-t_{2}} \circ \tau^{t_{2}}(A)-\tau_{V}^{-t_{1}} \circ \tau^{t_{1}}(A)=-\mathrm{i} \int_{t_{1}}^{t_{2}} \tau_{V}^{-t}\left(\left[V, \tau^{t}(A)\right]\right) \mathrm{d} t,
\end{aligned}
$$

and so

$$
\begin{aligned}
& \left\|\tau^{-t_{2}} \circ \tau_{V}^{t_{2}}(A)-\tau^{-t_{1}} \circ \tau_{V}^{t_{1}}(A)\right\| \leq \int_{t_{1}}^{t_{2}}\left\|\left[V, \tau_{V}^{t}(A)\right]\right\| \mathrm{d} t, \\
& \left\|\tau_{V}^{-t_{2}} \circ \tau^{t_{2}}(A)-\tau_{V}^{-t_{1}} \circ \tau^{t_{1}}(A)\right\| \leq \int_{t_{1}}^{t_{2}}\left\|\left[V, \tau^{t}(A)\right]\right\| \mathrm{d} t .
\end{aligned}
$$

To prove Part (i), note that (3.5) and the first estimate in (3.8) imply that for $A \in \mathcal{O}_{0}$ the norm limit

$$
\alpha_{V}^{+}(A) \equiv \lim _{t \rightarrow \infty} \tau^{-t} \circ \tau_{V}^{t}(A)
$$

exists. Since $\mathcal{O}_{0}$ is dense and $\tau^{-t} \circ \tau_{V}^{t}$ is isometric, the limit exists for all $A \in \mathcal{O}$, and $\alpha_{V}^{+}$is a $*$-morphism of $\mathcal{O}$. To prove Part (ii) note that the second estimate in (3.8) and (3.6) imply that the norm limit

$$
\beta_{V}^{+}(A) \equiv \lim _{t \rightarrow \infty} \tau_{V}^{-t} \circ \tau^{t}(A),
$$


also exists for all $A \in \mathcal{O}$. Since $\alpha_{V}^{+} \circ \beta_{V}^{+}(A)=A, \alpha_{V}^{+}$is a $*$-automorphism of $\mathcal{O} . \square$

Until the end of this subsection we will assume that the Assumption (S) holds and that $\omega$ is $\tau$-invariant.

Let $\tilde{\omega} \equiv \omega\left\lceil\mathcal{O}_{+}\right.$and let $\left(\mathcal{H}_{\tilde{\omega}}, \pi_{\tilde{\omega}}, \Omega_{\tilde{\omega}}\right)$ be the GNS-representation of $\mathcal{O}_{+}$associated to $\tilde{\omega}$. Obviously, if $\alpha_{V}^{+}$is an automorphism, then $\tilde{\omega}=\omega$. We denote by $\left(\mathcal{H}_{\omega_{+}}, \pi_{\omega_{+}}, \Omega_{\omega_{+}}\right)$the GNS representation of $\mathcal{O}$ associated to $\omega_{+}$. Let $L_{\tilde{\omega}}$ and $L_{\omega_{+}}$ be the standard Liouvilleans associated, respectively, to $\left(\mathcal{O}_{+}, \tau, \tilde{\omega}\right)$ and $\left(\mathcal{O}, \tau_{V}, \omega_{+}\right)$. Recall that $L_{\tilde{\omega}}$ is the unique self-adjoint operator on $\mathcal{H}_{\tilde{\omega}}$ such that for $A \in \mathcal{O}_{+}$,

$$
L_{\tilde{\omega}} \Omega_{\tilde{\omega}}=0, \quad \pi_{\tilde{\omega}}\left(\tau^{t}(A)\right)=\mathrm{e}^{\mathrm{i} t L_{\tilde{\omega}}} \pi_{\tilde{\omega}}(A) \mathrm{e}^{-\mathrm{i} t L_{\tilde{\omega}}},
$$

and similarly for $L_{\omega_{+}}$.

Proposition 3.8 The map

$$
U \pi_{\tilde{\omega}}\left(\alpha_{V}^{+}(A)\right) \Omega_{\tilde{\omega}}=\pi_{\omega_{+}}(A) \Omega_{\omega_{+}},
$$

extends to a unitary $U: \mathcal{H}_{\tilde{\omega}} \rightarrow \mathcal{H}_{\omega_{+}}$which intertwines $L_{\tilde{\omega}}$ and $L_{\omega_{+}}$, i.e.,

$$
U L_{\tilde{\omega}}=L_{\omega_{+}} U
$$

Proof. Set $\pi_{\tilde{\omega}}^{\prime}(A) \equiv \pi_{\tilde{\omega}}\left(\alpha_{V}^{+}(A)\right)$ and note that $\pi_{\tilde{\omega}}^{\prime}(\mathcal{O}) \Omega_{\tilde{\omega}}=\pi_{\tilde{\omega}}\left(\mathcal{O}_{+}\right) \Omega_{\tilde{\omega}}$, so that $\Omega_{\tilde{\omega}}$ is cyclic for $\pi_{\tilde{\omega}}^{\prime}(\mathcal{O})$. Since

$$
\omega_{+}(A)=\omega\left(\alpha_{V}^{+}(A)\right)=\tilde{\omega}\left(\alpha_{V}^{+}(A)\right)=\left(\Omega_{\tilde{\omega}}, \pi_{\tilde{\omega}}\left(\alpha_{V}^{+}(A)\right) \Omega_{\tilde{\omega}}\right)=\left(\Omega_{\tilde{\omega}}, \pi_{\tilde{\omega}}^{\prime}(A) \Omega_{\tilde{\omega}}\right),
$$

$\left(\mathcal{H}_{\tilde{\omega}}, \pi_{\tilde{\omega}}^{\prime}, \Omega_{\tilde{\omega}}\right)$ is also a GNS representation of $\mathcal{O}$ associated to $\omega_{+}$. Since GNS representations associated to the same state are unitarily equivalent, there is a unitary $U: \mathcal{H}_{\tilde{\omega}} \rightarrow \mathcal{H}_{\omega_{+}}$such that $U \Omega_{\tilde{\omega}}=\Omega_{\omega_{+}}$and

$$
U \pi_{\tilde{\omega}}^{\prime}(A)=\pi_{\omega_{+}}(A) U
$$

Finally, the identities

$$
\begin{aligned}
U \mathrm{e}^{\mathrm{i} t L_{\tilde{\omega}}} \pi_{\tilde{\omega}}^{\prime}(A) \Omega_{\tilde{\omega}} & =U \pi_{\tilde{\omega}}\left(\tau^{t}\left(\alpha_{V}^{+}(A)\right)\right) \Omega_{\tilde{\omega}}=U \pi_{\tilde{\omega}}\left(\alpha_{V}^{+}\left(\tau_{V}^{t}(A)\right)\right) \Omega_{\tilde{\omega}} \\
& =\pi_{\omega_{+}}\left(\tau_{V}^{t}(A)\right) \Omega_{\omega_{+}}=\mathrm{e}^{\mathrm{i} t L_{\omega_{+}}} \pi_{\omega_{+}}(A) \Omega_{\omega_{+}} \\
& =\mathrm{e}^{\mathrm{i} t L_{\omega_{+}}} U \pi_{\tilde{\omega}}^{\prime}(A) \Omega_{\tilde{\omega}},
\end{aligned}
$$

yield that $U$ intertwines $L_{\tilde{\omega}}$ and $L_{\omega^{+}}$.

We finish this subsection with:

Proposition 3.9 (i) Assume that $\tilde{\omega} \in E\left(\mathcal{O}_{+}, \tau\right)$ is $\tau$-ergodic. Then

$$
\Sigma_{+}\left(\eta, \tau_{V}\right)=\left\{\omega_{+}\right\},
$$

for all $\eta \in \mathcal{N}_{\omega}$. 
(ii) If $\tilde{\omega}$ is $\tau$-mixing, then

$$
\lim _{t \rightarrow \infty} \eta \circ \tau_{V}^{t}=\omega_{+},
$$

for all $\eta \in \mathcal{N}_{\omega}$.

Proof. We will prove the Part (i); the proof of the Part (ii) is similar. If $\eta \in \mathcal{N}_{\omega}$, then $\eta\left\lceil\mathcal{O}_{+} \in \mathcal{N}_{\tilde{\omega}}\right.$, and the ergodicity of $\tilde{\omega}$ yields

$$
\lim _{T \rightarrow \infty} \frac{1}{T} \int_{0}^{T} \eta\left(\tau^{t}\left(\alpha_{V}^{+}(A)\right)\right) \mathrm{d} t=\tilde{\omega}\left(\alpha_{V}^{+}(A)\right)=\omega_{+}(A) .
$$

This fact, the estimate

$$
\left\|\eta\left(\tau_{V}^{t}(A)\right)-\eta\left(\tau^{t}\left(\alpha_{V}^{+}(A)\right)\right)\right\| \leq\left\|\tau^{-t} \circ \tau_{V}^{t}(A)-\alpha_{V}^{+}(A)\right\|,
$$

and Assumption (S) yield the statement.

\section{Open quantum systems}

\subsection{Definition}

Open quantum systems are the basic paradigms of non-equilibrium quantum statistical mechanics. An open system consists of a "small" system $\mathcal{S}$ interacting with a large "environment" or "reservoir" $\mathcal{R}$.

In these lecture notes the small system will be a "quantum dot"-a quantum mechanical system with finitely many energy levels and no internal structure. The system $\mathcal{S}$ is described by a finite-dimensional Hilbert space $\mathcal{H}_{\mathcal{S}}=\mathbb{C}^{N}$ and a Hamiltonian $H_{\mathcal{S}}$. Its algebra of observables $\mathcal{O}_{\mathcal{S}}$ is the full matrix algebra $M_{N}(\mathbb{C})$ and its dynamics is given by

$$
\tau_{\mathcal{S}}^{t}(A)=\mathrm{e}^{\mathrm{i} t H_{\mathcal{S}}} A \mathrm{e}^{-\mathrm{i} t H_{\mathcal{S}}}=\mathrm{e}^{t \delta_{\mathcal{S}}}(A),
$$

where $\delta_{\mathcal{S}}(\cdot)=\mathrm{i}\left[H_{\mathcal{S}}, \cdot\right]$. The states of $\mathcal{S}$ are density matrices on $\mathcal{H}_{\mathcal{S}}$. A convenient reference state is the tracial state, $\omega_{\mathcal{S}}(\cdot)=\operatorname{Tr}(\cdot) / \operatorname{dim} \mathcal{H}_{\mathcal{S}}$. In the physics literature $\omega_{\mathcal{S}}$ is sometimes called the chaotic state since it is of maximal entropy, giving the same probability $1 / \operatorname{dim} \mathcal{H}_{\mathcal{S}}$ to any one-dimensional projection in $\mathcal{H}_{\mathcal{S}}$.

The reservoir is described by a $C^{*}$-dynamical system $\left(\mathcal{O}_{\mathcal{R}}, \tau_{\mathcal{R}}\right)$ and a reference state $\omega_{\mathcal{R}}$. We denote by $\delta_{\mathcal{R}}$ the generator of $\tau_{\mathcal{R}}$.

The algebra of observables of the joint system $\mathcal{S}+\mathcal{R}$ is $\mathcal{O}=\mathcal{O}_{\mathcal{S}} \otimes \mathcal{O}_{\mathcal{R}}$ and its reference state is $\omega \equiv \omega_{\mathcal{S}} \otimes \omega_{\mathcal{R}}$. Its dynamics, still decoupled, is given by $\tau^{t}=\tau_{\mathcal{S}}^{t} \otimes \tau_{\mathcal{R}}^{t}$. Let $V=V^{*} \in \mathcal{O}$ be a local perturbation which couples $\mathcal{S}$ to the reservoir $\mathcal{R}$. The $*$ derivation $\delta_{V} \equiv \delta_{\mathcal{R}}+\delta_{\mathcal{S}}+\mathrm{i}[V, \cdot]$ generates the coupled dynamics $\tau_{V}^{t}$ on $\mathcal{O}$. The coupled joint system $\mathcal{S}+\mathcal{R}$ is described by the $C^{*}$-dynamical system $\left(\mathcal{O}, \tau_{V}\right)$ and the reference state $\omega$. Whenever the meaning is clear within the context, we will identify $\mathcal{O}_{\mathcal{S}}$ and $\mathcal{O}_{\mathcal{R}}$ with subalgebras of $\mathcal{O}$ via $A \otimes I_{\mathcal{O}_{\mathcal{R}}}, I_{\mathcal{O}_{\mathcal{S}}} \otimes A$. With a slight abuse of notation, in the sequel we denote $I_{\mathcal{O}_{\mathcal{R}}}$ and $I_{\mathcal{O}_{\mathcal{S}}}$ by $I$.

We will suppose that the reservoir $\mathcal{R}$ has additional structure, namely that it consists of $M$ parts $\mathcal{R}_{1}, \cdots, \mathcal{R}_{M}$, which are interpreted as subreservoirs. The subreservoirs 
are assumed to be independent-they interact only through the small system which allows for the flow of energy and matter between various subreservoirs.

The subreservoir structure of $\mathcal{R}$ can be chosen in a number of different ways and the choice ultimately depends on the class of examples one wishes to describe. One obvious choice is the following: the $j$-th reservoir is described by the $C^{*}$-dynamical system $\left(\mathcal{O}_{\mathcal{R}_{j}}, \tau_{\mathcal{R}_{j}}\right)$ and the reference state $\omega_{\mathcal{R}_{j}}$, and $\mathcal{O}_{\mathcal{R}}=\otimes \mathcal{O}_{\mathcal{R}_{j}}, \tau_{\mathcal{R}}=\otimes \mathcal{\tau}_{\mathcal{R}_{j}}$, $\omega=\otimes \omega_{\mathcal{R}_{j}}[\mathrm{JP} 4$, Ru1]. In view of the examples we plan to cover, we will choose a more general subreservoir structure.

We will assume that the $j$-th reservoir is described by a $C^{*}$-subalgebra $\mathcal{O}_{\mathcal{R}_{j}} \subset \mathcal{O}_{\mathcal{R}}$ which is preserved by $\tau_{\mathcal{R}}$. We denote the restrictions of $\tau_{\mathcal{R}}$ and $\omega_{\mathcal{R}}$ to $\mathcal{O}_{\mathcal{R}_{j}}$ by $\tau_{\mathcal{R}_{j}}$ and $\omega_{\mathcal{R}_{j}}$. Different algebras $\mathcal{O}_{\mathcal{R}_{j}}$ may not commute. However, we will assume that $\mathcal{O}_{\mathcal{R}_{i}} \cap \mathcal{O}_{\mathcal{R}_{j}}=\mathbb{C} I$ for $i \neq j$. If $\mathcal{A}_{k}, 1 \leq k \leq N$, are subsets of $\mathcal{O}_{\mathcal{R}}$, we denote by $\left\langle\mathcal{A}_{1}, \cdots, \mathcal{A}_{N}\right\rangle$ the minimal $C^{*}$-subalgebra of $\mathcal{O}_{\mathcal{R}}$ that contains all $\mathcal{A}_{k}$. Without loss of generality, we may assume that $\mathcal{O}_{\mathcal{R}}=\left\langle\mathcal{O}_{\mathcal{R}_{1}}, \cdots, \mathcal{O}_{\mathcal{R}_{M}}\right\rangle$.

The system $\mathcal{S}$ is coupled to the reservoir $\mathcal{R}_{j}$ through a junction described by a self-adjoint perturbation $V_{j} \in \mathcal{O}_{\mathcal{S}} \otimes \mathcal{O}_{\mathcal{R}_{j}}$. The complete interaction is given by

$$
V \equiv \sum_{j=1}^{M} V_{j} .
$$

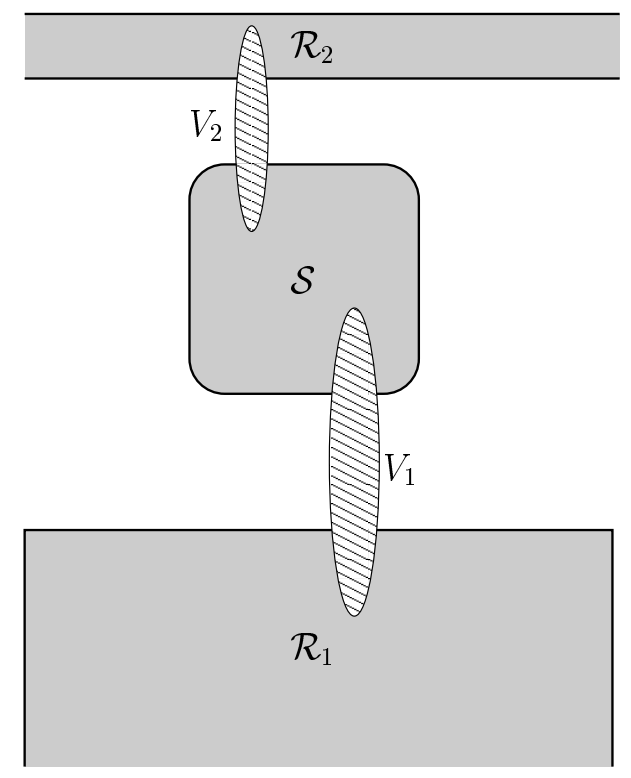

Figure 1: Junctions $V_{1}, V_{2}$ between the system $\mathcal{S}$ and subreservoirs.

An anti-linear, involutive, $*$-automorphism $\mathfrak{r}: \mathcal{O} \rightarrow \mathcal{O}$ is called a time reversal if it 
satisfies $\mathfrak{r}\left(H_{\mathcal{S}}\right)=H_{\mathcal{S}}, \mathfrak{r}\left(V_{j}\right)=V_{j}$ and $\mathfrak{r} \circ \tau_{\mathcal{R}_{j}}^{t}=\tau_{\mathcal{R}_{j}}^{-t} \circ \mathfrak{r}$. If $\mathfrak{r}$ is a time reversal, then

$$
\mathfrak{r} \circ \tau^{t}=\tau^{-t} \circ \mathfrak{r}, \quad \mathfrak{r} \circ \tau_{V}^{t}=\tau_{V}^{-t} \circ \mathfrak{r},
$$

and a state $\omega$ on $\mathcal{O}$ is time reversal invariant if $\omega \circ \mathfrak{r}(A)=\omega\left(A^{*}\right)$ for all $A \in \mathcal{O}$. An open quantum system described by $\left(\mathcal{O}, \tau_{V}\right)$ and the reference state $\omega$ is called time reversal invariant (TRI) if there exists a time reversal $\mathfrak{r}$ such that $\omega$ is time reversal invariant.

\section{2 $C^{*}$-scattering for open quantum systems}

Except for Part (ii) of Proposition 3.7, the scattering approach to the study of NESS, described in Subsection 3.4, is directly applicable to open quantum systems. Concerning Part (ii) of Proposition 3.7, note that in the case of open quantum systems the Møller morphism $\alpha_{V}^{+}$cannot be onto (except in trivial cases). The best one may hope for is that $\mathcal{O}_{+}=\mathcal{O}_{\mathcal{R}}$, namely that $\alpha_{V}^{+}$is an isomorphism between the $C^{*}$-dynamical systems $\left(\mathcal{O}, \tau_{V}\right)$ and $\left(\mathcal{O}_{\mathcal{R}}, \tau_{\mathcal{R}}\right)$. The next theorem was proved in [Ru1].

Theorem 4.1 Suppose that Assumption (S) holds.

(i) If there exists a dense set $\mathcal{O}_{\mathcal{R} 0} \subset \mathcal{O}_{\mathcal{R}}$ such that for all $A \in \mathcal{O}_{\mathcal{R} 0 \text {, }}$,

$$
\int_{0}^{\infty}\left\|\left[V, \tau^{t}(A)\right]\right\| \mathrm{d} t<\infty
$$

then $\mathcal{O}_{\mathcal{R}} \subset \mathcal{O}_{+}$.

(ii) If there exists a dense set $\mathcal{O}_{0} \subset \mathcal{O}$ such that for all $X \in \mathcal{O}_{\mathcal{S}}$ and $A \in \mathcal{O}_{0}$,

$$
\lim _{t \rightarrow+\infty}\left\|\left[X, \tau_{V}^{t}(A)\right]\right\|=0,
$$

then $\mathcal{O}_{+} \subset \mathcal{O}_{\mathcal{R}}$.

(iii) If both (4.10) and (4.11) hold then $\alpha_{V}^{+}$is an isomorphism between the $C^{*}$-dynamical systems $\left(\mathcal{O}, \tau_{V}\right)$ and $\left(\mathcal{O}_{\mathcal{R}}, \tau_{\mathcal{R}}\right)$. In particular, if $\omega_{\mathcal{R}}$ is a $\left(\tau_{\mathcal{R}}, \beta\right)-K M S$ for some inverse temperature $\beta$, then $\omega_{+}$is a $\left(\tau_{V}, \beta\right)-K M S$ state.

Proof. The proof of Part (i) is similar to the proof of the Part (i) of Proposition 3.7. The assumption (4.10) ensures that the limits

$$
\beta_{V}^{+}(A)=\lim _{t \rightarrow \infty} \tau_{V}^{t} \circ \tau^{-t}(A),
$$

exist for all $A \in \mathcal{O}_{\mathcal{R}}$. Clearly, $\alpha_{V}^{+} \circ \beta_{V}^{+}(A)=A$ for all $A \in \mathcal{O}_{\mathcal{R}}$ and so $\mathcal{O}_{\mathcal{R}} \subset \operatorname{Ran} \alpha_{V}^{+}$.

To prove Part (ii) recall that $\mathcal{O}_{\mathcal{S}}$ is a $N^{2}$-dimensional matrix algebra. It has a basis $\left\{E_{k} \mid k=1, \cdots, N^{2}\right\}$ such that $\tau^{t}\left(E_{k}\right)=\mathrm{e}^{\mathrm{i} t \theta_{k}} E_{k}$ for some $\theta_{k} \in \mathbb{R}$. From Assumption (S) and (4.11) we can conclude that

$$
0=\lim _{t \rightarrow+\infty} \mathrm{e}^{\mathrm{i} t \theta_{k}} \tau^{-t}\left(\left[E_{k}, \tau_{V}^{t}(A)\right]\right)=\lim _{t \rightarrow+\infty}\left[E_{k}, \tau^{-t} \circ \tau_{V}^{t}(A)\right]=\left[E_{k}, \alpha_{V}^{+}(A)\right],
$$

for all $A \in \mathcal{O}_{0}$ and hence, by continuity, for all $A \in \mathcal{O}$. It follows that $\operatorname{Ran} \alpha_{V}^{+}$belongs to the commutant of $\mathcal{O}_{\mathcal{S}}$ in $\mathcal{O}$. Since $\mathcal{O}$ can be seen as the algebra $M_{N}\left(\mathcal{O}_{\mathcal{R}}\right)$ of $N \times N$ matrices with entries in $\mathcal{O}_{\mathcal{R}}$, one easily checks that this commutant is precisely $\mathcal{O}_{\mathcal{R}}$.

Part (iii) is a direct consequence of the first two parts. 


\subsection{The first and second law of thermodynamics}

Let us denote by $\delta_{j}$ the generator of the dynamical group $\tau_{\mathcal{R}_{j}}$. (Recall that this dynamical group is the restriction of the decoupled dynamics to the subreservoir $\mathcal{R}_{j}$ ). Assume that $V_{j} \in \operatorname{Dom}\left(\delta_{j}\right)$. The generator of $\tau_{V}$ is $\delta_{V}=\delta_{\mathcal{R}}+\mathrm{i}\left[H_{\mathcal{S}}+V, \cdot\right]$ and it follows from (4.9) that the total energy flux out of the reservoir is given by

$$
\frac{d}{d t} \tau_{V}^{t}\left(H_{\mathcal{S}}+V\right)=\tau_{V}^{t}\left(\delta_{V}\left(H_{\mathcal{S}}+V\right)\right)=\tau_{V}^{t}\left(\delta_{\mathcal{R}}(V)\right)=\sum_{j=1}^{M} \tau_{V}^{t}\left(\delta_{j}\left(V_{j}\right)\right) .
$$

Thus, we can identify the observable describing the heat flux out of the $j$-th reservoir as

$$
\Phi_{j}=\delta_{j}(V)=\delta_{j}\left(V_{j}\right)=\delta_{\mathcal{R}}\left(V_{j}\right) .
$$

We note that if $\mathfrak{r}$ is a time-reversal, then $\mathfrak{r}\left(\Phi_{j}\right)=-\Phi_{j}$. The energy balance equation

$$
\sum_{j=1}^{M} \Phi_{j}=\delta_{V}\left(H_{\mathcal{S}}+V\right)
$$

yields the conservation of energy (the first law of thermodynamics): for any $\tau_{V}$-invariant state $\eta$,

$$
\sum_{j=1}^{M} \eta\left(\Phi_{j}\right)=0
$$

Besides heat fluxes, there might be other fluxes across the system $\mathcal{S}+\mathcal{R}$ (for example, matter and charge currents). We will not discuss here the general theory of such fluxes (the related information can be found in [FMU, FMSU, TM]). In the rest of this section we will focus on the thermodynamics of heat fluxes. Charge currents will be discussed in the context of a concrete model in the second part of this lecture.

We now turn to the entropy production. Assume that there exists a $C^{*}$-dynamics $\sigma_{\mathcal{R}}^{t}$ on $\mathcal{O}_{\mathcal{R}}$ such that $\omega_{\mathcal{R}}$ is $\left(\sigma_{\mathcal{R}},-1\right)$-KMS state and such that $\sigma_{\mathcal{R}}$ preserves each subalgebra $\mathcal{O}_{\mathcal{R}_{j}}$. Let $\tilde{\delta}_{j}$ be the generator of the restriction of $\sigma_{\mathcal{R}}$ to $\mathcal{O}_{\mathcal{R}_{j}}$ and assume that $V_{j} \in \operatorname{Dom}\left(\tilde{\delta}_{j}\right)$. The entropy production observable associated to the perturbation $V$ and the reference state $\omega=\omega_{\mathcal{S}} \otimes \omega_{\mathcal{R}}$, where $\omega_{\mathcal{S}}(\cdot)=\operatorname{Tr}(\cdot) / \operatorname{dim} \mathcal{H}_{\mathcal{S}}$, is

$$
\sigma_{V}=\sum_{j=1}^{M} \tilde{\delta}_{j}\left(V_{j}\right)
$$

Until the end of this section we shall assume that the reservoirs $\mathcal{O}_{\mathcal{R}_{j}}$ are in thermal equilibrium at inverse temperatures $\beta_{j}$. More precisely, we will assume that $\omega_{\mathcal{R}_{j}}$ is the unique $\left(\tau_{\mathcal{R}_{j}}, \beta_{j}\right)$-KMS state on $\mathcal{O}_{\mathcal{R}_{j}}$. Then $\tilde{\delta}_{j}=-\beta_{j} \delta_{j}$, and

$$
\sigma_{V}=-\sum_{j=1}^{M} \beta_{j} \Phi_{j}
$$


In particular, for any NESS $\omega_{+} \in \Sigma_{+}\left(\omega, \tau_{V}\right)$, the second law of thermodynamics holds:

$$
\sum_{j=1}^{M} \beta_{j} \omega_{+}\left(\Phi_{j}\right)=-\operatorname{Ep}\left(\omega_{+}\right) \leq 0 .
$$

In fact, it is not difficult to show that $\operatorname{Ep}\left(\omega_{+}\right)$is independent of the choice of the reference state of the small system as long as $\omega_{\mathcal{S}}>0$; see Proposition 5.3 in [JP4]. In the case of two reservoirs, the relation

$$
\left(\beta_{1}-\beta_{2}\right) \omega_{+}\left(\Phi_{1}\right)=\beta_{1} \omega_{+}\left(\Phi_{1}\right)+\beta_{2} \omega_{+}\left(\Phi_{2}\right) \leq 0,
$$

yields that the heat flows from the hot to the cold reservoir.

\subsection{Linear response theory}

Linear response theory describes thermodynamics in the regime where the "forces" driving the system out of equilibrium are weak. In such a regime, to a very good approximation, the non-equilibrium currents depend linearly on the forces. The ultimate purpose of linear response theory is to justify well known phenomenological laws like Ohm's law for charge currents or Fick's law for heat currents. We are still far from a satisfactory derivation of these laws, even in the framework of classical mechanics; see [BLR] for a recent review on this matter. We also refer to [GVV6] for a rigorous discussion of linear response theory at the macroscopic level.

A less ambitious application of linear response theory concerns transport properties of microscopic and mesoscopic quantum devices (the advances in nanotechnologies during the last decade have triggered a strong interest in the transport properties of such devices). Linear response theory of such systems is much better understood, as we shall try to illustrate.

In our current setting, the forces that drive the system $\mathcal{S}+\mathcal{R}$ out of equilibrium are the different inverse temperatures $\beta_{1}, \cdots, \beta_{M}$ of the reservoirs attached to $\mathcal{S}$. If all inverse temperatures $\beta_{j}$ are sufficiently close to some value $\beta_{\text {eq }}$, we expect linear response theory to give a good account of the thermodynamics of the system near thermal equilibrium at inverse temperature $\beta_{\text {eq }}$.

To emphasize the fact that the reference state $\omega=\omega_{\mathcal{S}} \otimes \omega_{\mathcal{R}}$ depends on the $\beta_{j}$ we set $X=\left(X_{1}, \cdots, X_{M}\right)$ with $X_{j} \equiv \beta_{\text {eq }}-\beta_{j}$ and denote by $\omega_{X}$ this reference state. We assume that for some $\epsilon>0$ and all $|X|<\epsilon$ there exists a unique NESS $\omega_{X+} \in \Sigma_{+}\left(\omega_{X}, \tau_{V}\right)$ and that the functions $X \mapsto \omega_{X+}\left(\Phi_{j}\right)$ are $C^{2}$. Note that $\omega_{0+}$ is the (unique) $\left(\tau_{V}, \beta_{\text {eq }}\right)$-KMS state on $\mathcal{O}$. We will denote it simply by $\omega_{\beta_{\text {eq }}}$.

In phenomenological non-equilibrium thermodynamics, the duality between the driving forces $F_{\alpha}$, also called affinities, and the steady currents $\phi_{\alpha}$ they induce is expressed by the entropy production formula

$$
\mathrm{Ep}=\sum_{\alpha} F_{\alpha} \phi_{\alpha}
$$

(see [DGM]). The steady currents are themselves functions of the affinities $\phi_{\alpha}=$ 
$\phi_{\alpha}\left(F_{1}, \cdots\right)$. In the linear response regime, these functions are given by the relations

$$
\phi_{\alpha}=\sum_{\gamma} L_{\alpha \gamma} F_{\gamma}
$$

which define the kinetic coefficients $L_{\alpha \gamma}$.

Comparing with Equ. (4.13) and using energy conservation (4.12) we obtain in our case

$$
\operatorname{Ep}\left(\omega_{X+}\right)=\sum_{j=1}^{M} X_{j} \omega_{X+}\left(\Phi_{j}\right) .
$$

Thus $X_{j}$ is the affinity conjugated to the steady heat flux $\phi_{j}(X)=\omega_{X+}\left(\Phi_{j}\right)$ out of $\mathcal{R}_{j}$. We note in particular that the equilibrium entropy production vanishes. The kinetic coefficients $L_{j i}$ are given by

$$
L_{j i} \equiv\left(\frac{\partial \phi_{j}}{\partial X_{i}}\right)_{X=0}=\left.\partial_{X_{i}} \omega_{X+}\left(\Phi_{j}\right)\right|_{X=0}
$$

Taylor's formula yields

$$
\begin{gathered}
\phi_{j}(X)=\omega_{X+}\left(\Phi_{j}\right)=\sum_{i=1}^{M} L_{j i} X_{i}+O\left(\epsilon^{2}\right) \\
\operatorname{Ep}\left(\omega_{X+}\right)=\sum_{i, j=1}^{M} L_{j i} X_{i} X_{j}+o\left(\epsilon^{2}\right) .
\end{gathered}
$$

Combining (4.14) with the first law of thermodynamics (recall (4.12)) we obtain that for all $i$,

$$
\sum_{j=1}^{M} L_{j i}=0 .
$$

Similarly, (4.15) and the second law (4.13) imply that the quadratic form

$$
\sum_{i, j=1}^{M} L_{j i} X_{i} X_{j}
$$

on $\mathbb{R}^{M}$ is non-negative. Note that this does not imply that the $M \times M$-matrix $L$ is symmetric !

Linear response theory goes far beyond the above elementary relations. Its true cornerstones are the Onsager reciprocity relations (ORR), the Kubo fluctuation-dissipation formula (KF) and the Central Limit Theorem (CLT). All three of them deal with the kinetic coefficients. The Onsager reciprocity relations assert that the matrix $L_{j i}$ of a time reversal invariant (TRI) system is symmetric,

$$
L_{j i}=L_{i j} .
$$


For non-TRI systems, similar relations hold between the transport coefficients of the system and those of the time reversed one. For example, if time reversal invariance is broken by the action of an external magnetic field $B$, then the Onsager-Casimir relations

$$
L_{j i}(B)=L_{i j}(-B),
$$

hold.

The Kubo fluctuation-dissipation formula expresses the transport coefficients of a TRI system in terms of the equilibrium current-current correlation function

$$
C_{j i}(t) \equiv \frac{1}{2} \omega_{\beta_{\mathrm{eq}}}\left(\tau_{V}^{t}\left(\Phi_{j}\right) \Phi_{i}+\Phi_{i} \tau_{V}^{t}\left(\Phi_{j}\right)\right),
$$

namely

$$
L_{j i}=\frac{1}{2} \int_{-\infty}^{\infty} C_{j i}(t) \mathrm{d} t .
$$

The Central Limit Theorem further relates $L_{j i}$ to the statistics of the current fluctuations in equilibrium. In term of characteristic function, the CLT for open quantum systems in thermal equilibrium asserts that

$$
\lim _{t \rightarrow \infty} \omega_{\beta_{\text {eq }}}\left(\mathrm{e}^{\mathrm{i}\left(\sum_{j=1}^{M} \xi_{j} \int_{0}^{t} \tau_{V}^{s}\left(\Phi_{j}\right) \mathrm{d} s\right) / \sqrt{t}}\right)=\mathrm{e}^{-\frac{1}{2} \sum_{i, j=1}^{M} D_{j i} \xi_{j} \xi_{i}},
$$

where the covariance matrix $D_{j i}$ is given by

$$
D_{j i}=2 L_{j i}
$$

If, for a self-adjoint $A \in \mathcal{O}$, we denote by $1_{[a, b]}(A)$ the spectral projection on the interval $[a, b]$ of $\pi_{\omega_{\beta_{\text {eq }}}}(A)$, the probability of measuring a value of $A$ in $[a, b]$ when the system is in the state $\omega_{\beta_{\mathrm{eq}}}$ is given by

$$
\operatorname{Prob}_{\omega_{\beta_{\mathrm{eq}}}}\{A \in[a, b]\}=\left(\Omega_{\omega_{\beta_{\mathrm{eq}}}}, 1_{[a, b]}(A) \Omega_{\omega_{\beta_{\mathrm{eq}}}}\right) .
$$

It then follows from (4.20) that

$$
\lim _{t \rightarrow \infty} \operatorname{Prob}_{\omega_{\beta_{\mathrm{eq}}}}\left\{\frac{1}{t} \int_{0}^{t} \tau_{V}^{s}\left(\Phi_{j}\right) \mathrm{d} s \in\left[\frac{a}{\sqrt{t}}, \frac{b}{\sqrt{t}}\right]\right\}=\frac{1}{\sqrt{2 \pi} L_{j j}} \int_{a}^{b} \mathrm{e}^{-x^{2} / 2 L_{j j}^{2}} \mathrm{~d} x .
$$

This is a direct translation to quantum mechanics of the classical central limit theorem. Because fluxes do not commute, $\left[\Phi_{j}, \Phi_{i}\right] \neq 0$ for $j \neq i$, they can not be measured simultaneously and a simple classical probabilistic interpretation of (4.20) for the vector variable $\Phi=\left(\Phi_{1}, \cdots, \Phi_{M}\right)$ is not possible. Instead, the quantum fluctuations of the vector variable $\Phi$ are described by the so-called fluctuation algebra [GVV1, GVV2, GVV3, GVV4, GVV5, Ma]. The description and study of the fluctuation algebra involve somewhat advanced technical tools and for this reason we will not discuss the quantum CLT theorem in this lecture.

The mathematical theory of ORR, KF, and CLT is reasonably well understood in classical statistical mechanics (see the lecture [Re]). In the context of open quantum systems these important notions are still not completely understood (see however [AJPP, JPR2] for some recent results). 
We close this subsection with some general comments about ORR and KF.

The definition (4.18) of the current-current correlation function involves a symmetrized product in order to ensure that the function $C_{j i}(t)$ is real-valued. The corresponding imaginary part, given by

$$
\frac{1}{2} \mathrm{i}\left[\Phi_{i}, \tau_{V}^{t}\left(\Phi_{j}\right)\right]
$$

is usually non-zero. However, since $\omega_{\beta_{\mathrm{eq}}}$ is a KMS state, the stability condition (see [BR2]) yields

$$
\int_{-\infty}^{\infty} \omega_{\beta_{\text {eq }}}\left(\mathrm{i}\left[\Phi_{i}, \tau_{V}^{t}\left(\Phi_{j}\right)\right]\right) \mathrm{d} t=0,
$$

so that, in this case, the symmetrization is not necessary and one can rewrite KF as

$$
L_{j i}=\frac{1}{2} \int_{-\infty}^{\infty} \omega_{\beta_{\mathrm{eq}}}\left(\Phi_{i} \tau_{V}^{t}\left(\Phi_{j}\right)\right) \mathrm{d} t
$$

Finally, we note that ORR follow directly from KF under the TRI assumption. Indeed, if our system is TRI with time reversal $\mathfrak{r}$ we have

$$
\mathfrak{r}\left(\Phi_{i}\right)=-\Phi_{i}, \quad \mathfrak{r}\left(\tau_{V}^{t}\left(\Phi_{j}\right)\right)=-\tau_{V}^{-t}\left(\Phi_{j}\right), \quad \omega_{\beta_{\mathrm{eq}}} \circ \mathfrak{r}=\omega_{\beta_{\mathrm{eq}}},
$$

and therefore

$$
C_{j i}(t)=\frac{1}{2} \omega_{\beta_{\mathrm{eq}}}\left(\tau_{V}^{-t}\left(\Phi_{j}\right) \Phi_{i}+\Phi_{i} \tau_{V}^{-t}\left(\Phi_{j}\right)\right)=C_{j i}(-t) .
$$

Since $\omega_{\beta_{\text {eq }}}$ is $\tau_{V}$-invariant, this implies

$$
C_{j i}(t)=\frac{1}{2} \omega_{\beta_{\mathrm{eq}}}\left(\Phi_{j} \tau_{V}^{t}\left(\Phi_{i}\right)+\tau_{V}^{t}\left(\Phi_{i}\right) \Phi_{j}\right)=C_{i j}(t)
$$

and ORR (4.17) follows from KF (4.19).

In the second part of the lecture we will show that the Onsager relations and the Kubo formula hold for the SEBB model. The proof of the Central Limit Theorem for this model is somewhat technically involved and can be found in [AJPP].

\subsection{Fermi Golden Rule (FGR) thermodynamics}

Let $\lambda \in \mathbb{R}$ be a control parameter. We consider an open quantum system with coupling $\lambda V$ and write $\tau_{\lambda}$ for $\tau_{\lambda V}, \omega_{\lambda+}$ for $\omega_{+}$, etc.

The NESS and thermodynamics of the system can be described, to second order of perturbation theory in $\lambda$, using the weak coupling (or van Hove) limit. This approach is much older than the "microscopic" Hamiltonian approach discussed so far, and has played an important role in the development of the subject. The classical references are [Da1, Da2, Haa, VH1, VH2, VH3]. The weak coupling limit is also discussed in the lecture notes [D1].

In the weak coupling limit one "integrates" the degrees of freedom of the reservoirs and follows the reduced dynamics of $\mathcal{S}$ on a large time scale $t / \lambda^{2}$. In the limit $\lambda \rightarrow 0$ 
the dynamics of $\mathcal{S}$ becomes irreversible and is described by a semigroup, often called the quantum Markovian semigroup (QMS). The generator of this QMS describes the thermodynamics of the open quantum system to second order of perturbation theory.

The "integration" of the reservoir variables is performed as follows. As usual, we use the injection $A \mapsto A \otimes I$ to identify $\mathcal{O}_{\mathcal{S}}$ with a subalgebra of $\mathcal{O}$. For $A \in \mathcal{O}_{\mathcal{S}}$ and $B \in \mathcal{O}_{\mathcal{R}}$ we set

$$
P_{\mathcal{S}}(A \otimes B)=A \omega_{\mathcal{R}}(B)
$$

The map $P_{\mathcal{S}}$ extends to a projection $P_{\mathcal{S}}: \mathcal{O} \rightarrow \mathcal{O}_{\mathcal{S}}$. The reduced dynamics of the system $\mathcal{S}$ is described by the family of maps $T_{\lambda}^{t}: \mathcal{O}_{\mathcal{S}} \rightarrow \mathcal{O}_{\mathcal{S}}$ defined by

$$
T_{\lambda}^{t}(A) \equiv P_{\mathcal{S}}\left(\tau_{0}^{-t} \circ \tau_{\lambda}^{t}(A \otimes I)\right) .
$$

Obviously, $T_{\lambda}^{t}$ is neither a group nor a semigroup. Let $\omega_{\mathcal{S}}$ be an arbitrary reference state (density matrix) of the small system and $\omega=\omega_{\mathcal{S}} \otimes \omega_{\mathcal{R}}$. Then for any $A \in \mathcal{O}_{\mathcal{S}}$,

$$
\omega\left(\tau_{0}^{-t} \circ \tau_{\lambda}^{t}(A \otimes I)\right)=\operatorname{Tr}_{\mathcal{H}_{\mathcal{S}}}\left(\omega_{\mathcal{S}} T_{\lambda}^{t}(A)\right) .
$$

In [Da1, Da2] Davies proved that under very general conditions there exists a linear map $K_{\mathrm{H}}: \mathcal{O}_{\mathcal{S}} \rightarrow \mathcal{O}_{\mathcal{S}}$ such that

$$
\lim _{\lambda \rightarrow 0} T_{\lambda}^{t / \lambda^{2}}(A)=\mathrm{e}^{t K_{\mathrm{H}}}(A) .
$$

The operator $K_{\mathrm{H}}$ is the QMS generator (sometimes called the Davies generator) in the Heisenberg picture. A substantial body of literature has been devoted to the study of the operator $K_{\mathrm{H}}$ (see the lecture notes [D1]). Here we recall only a few basic results concerning thermodynamics in the weak coupling limit (for additional information see [LeSp]). We will assume that the general conditions described in the lecture notes [D1] are satisfied.

The operator $K_{\mathrm{H}}$ generates a positivity preserving contraction semigroup on $\mathcal{O}_{\mathcal{S}}$. Obviously, $K_{\mathrm{H}}(I)=0$. We will assume that zero is the only purely imaginary eigenvalue of $K_{\mathrm{H}}$ and that $\operatorname{Ker} K_{\mathrm{H}}=\mathbb{C} I$. This non-degeneracy condition can be naturally characterized in algebraic terms, see [D1, Sp]. It implies that the eigenvalue 0 of $K_{\mathrm{H}}$ is semi-simple, that the corresponding eigenprojection has the form $A \mapsto \operatorname{Tr}\left(\omega_{\mathcal{S}}+A\right) I$, where $\omega_{\mathcal{S}+}$ is a density matrix, and that for any initial density matrix $\omega_{\mathcal{S}}$,

$$
\lim _{t \rightarrow \infty} \operatorname{Tr}\left(\omega_{\mathcal{S}} \mathrm{e}^{t K_{\mathrm{H}}}(A)\right)=\operatorname{Tr}\left(\omega_{\mathcal{S}} A\right) \equiv \omega_{\mathcal{S}+}(A) .
$$

The density matrix $\omega_{\mathcal{S}}+$ describes the NESS of the open quantum system in the weak coupling limit. One further shows that the operator $K_{\mathrm{H}}$ has the form

$$
K_{\mathrm{H}}=\sum_{j=1}^{M} K_{\mathrm{H}, j},
$$

where $K_{\mathrm{H}, j}$ is the QMS generator obtained by considering the weak coupling limit of the coupled system $\mathcal{S}+\mathcal{R}_{j}$, i.e.,

$$
\mathrm{e}^{t K_{\mathrm{H}, j}}(A)=\lim _{\lambda \rightarrow 0} P_{\mathcal{S}}\left(\tau_{0}^{-t / \lambda^{2}} \circ \tau_{\lambda, j}^{t / \lambda^{2}}(A \otimes I)\right),
$$


where $\tau_{\lambda, j}$ is generated by $\delta_{j}+\mathrm{i}\left[H_{\mathcal{S}}+\lambda V_{j}, \cdot\right]$.

One often considers the QMS generator in the Schrödinger picture, denoted $K_{\mathrm{S}}$. The operator $K_{\mathrm{S}}$ is the adjoint of $K_{\mathrm{H}}$ with respect to the inner product $(X, Y)=$ $\operatorname{Tr}\left(X^{*} Y\right)$. The semigroup $\mathrm{e}^{t K_{\mathrm{S}}}$ is positivity and trace preserving. One similarly defines $K_{\mathrm{S}, j}$. Obviously,

$$
K_{\mathrm{S}}\left(\omega_{\mathcal{S}+}\right)=0, \quad K_{\mathrm{S}}=\sum_{j=1}^{M} K_{\mathrm{S}, j} .
$$

Recall our standing assumption that the reservoirs $\mathcal{O}_{\mathcal{R}_{j}}$ are in thermal equilibrium at inverse temperature $\beta_{j}$. We denote by

$$
\omega_{\beta}=\mathrm{e}^{-\beta H_{\mathcal{S}}} / \operatorname{Tr}\left(\mathrm{e}^{-\beta H_{\mathcal{S}}}\right),
$$

the canonical density matrix of $\mathcal{S}$ at inverse temperature $\beta$ (the unique $\left(\tau_{\mathcal{S}}, \beta\right.$ )-KMS state on $\mathcal{O}_{\mathcal{S}}$ ). Araki's perturbation theory of KMS-states (see [DJP, BR2]) yields that for $A \in \mathcal{O}_{\mathcal{S}}$,

$$
\omega_{\beta_{j}} \otimes \omega_{\mathcal{R}_{j}}\left(\tau_{0}^{-t} \circ \tau_{\lambda, j}^{t}(A \otimes I)\right)=\omega_{\beta_{j}}(A)+O(\lambda),
$$

uniformly in $t$. Hence, for all $t \geq 0$,

$$
\omega_{\beta_{j}}\left(\mathrm{e}^{t K_{\mathrm{H}, j}}(A)\right)=\omega_{\beta_{j}}(A),
$$

and so $K_{\mathrm{S}, j}\left(\omega_{\beta_{j}}\right)=0$. In particular, if all $\beta_{j}$ 's are the same and equal to $\beta$, then $\omega_{\mathcal{S}+}=\omega_{\beta}$.

Let $\mathcal{O}_{\mathrm{d}} \subset \mathcal{O}_{\mathcal{S}}$ be the $*$-algebra spanned by the eigenprojections of $H_{\mathcal{S}} . \mathcal{O}_{\mathrm{d}}$ is commutative and preserved by $K_{\mathrm{H}}, K_{\mathrm{H}, j}, K_{\mathrm{S}}$ and $K_{\mathrm{S}, j}$ [D1]. The NESS $\omega_{\mathcal{S}+}$ commutes with $H_{\mathcal{S}}$. If the eigenvalues of $H_{\mathcal{S}}$ are simple, then the restriction $K_{\mathrm{H}} \uparrow \mathcal{O}_{\mathrm{d}}$ is a generator of a Markov process whose state space is the spectrum of $H_{\mathcal{S}}$. This process has played an important role in the early development of quantum field theory (more on this in Subsection 8.2).

We now turn to the thermodynamics in the weak coupling limit, which we will call Fermi Golden Rule (FGR) thermodynamics. The observable describing the heat flux out of the $j$-th reservoir is

$$
\Phi_{\mathrm{fgr}, j}=K_{\mathrm{H}, j}\left(H_{\mathcal{S}}\right) .
$$

Note that $\Phi_{\mathrm{fgr}, j} \in \mathcal{O}_{\mathrm{d}}$. Since $K_{\mathrm{S}}\left(\omega_{\mathcal{S}+}\right)=0$ we have

$$
\sum_{j=1}^{M} \omega_{\mathcal{S}+}\left(\Phi_{\mathrm{fgr}, j}\right)=\omega_{\mathcal{S}+}\left(K_{\mathrm{H}}\left(H_{\mathcal{S}}\right)\right)=0,
$$

which is the first law of FGR thermodynamics.

The entropy production observable is

$$
\sigma_{\mathrm{fgr}}=-\sum_{j=1}^{M} \beta_{j} \Phi_{\mathrm{fgr}, j},
$$


and the entropy production of the NESS $\omega_{\mathcal{S}+}$ is

$$
\operatorname{Ep}_{\mathrm{fgr}}\left(\omega_{\mathcal{S}+}\right)=\omega_{\mathcal{S}+}\left(\sigma_{\mathrm{fgr}}\right)
$$

Since the semigroup generated by $K_{\mathrm{S}, j}$ is trace-preserving we have

$$
\left.\frac{\mathrm{d}}{\mathrm{d} t} \operatorname{Ent}\left(\mathrm{e}^{t K_{\mathrm{S}, j}} \omega_{\mathcal{S}}+\omega_{\beta_{j}}\right)\right|_{t=0}=-\beta_{j} \omega_{\mathcal{S}+}\left(\Phi_{\mathrm{fgr}, j}\right)-\operatorname{Tr}\left(K_{\mathrm{S}, j}\left(\omega_{\mathcal{S}+}\right) \log \omega_{\mathcal{S}+}\right),
$$

where the relative entropy is defined by (3.2). The function

$$
t \mapsto \operatorname{Ent}\left(\mathrm{e}^{t K_{\mathrm{S}, j}} \omega_{\mathcal{S}} \mid \omega_{\beta_{j}}\right),
$$

is non-decreasing (see [Li]), and so

$$
\operatorname{Ep}_{\mathrm{fgr}}\left(\omega_{\mathcal{S}+}\right)=\left.\sum_{j=1}^{M} \frac{\mathrm{d}}{\mathrm{d} t} \operatorname{Ent}\left(\mathrm{e}^{t K_{\mathrm{S}, j}} \omega_{\mathcal{S}+} \mid \omega_{\beta_{j}}\right)\right|_{t=0} \geq 0
$$

which is the second law of FGR thermodynamics. Moreover, under the usual nondegeneracy assumptions, $\operatorname{Ep}_{\mathrm{fgr}}\left(\omega_{\mathcal{S}+}\right)=0$ if and only if $\beta_{1}=\cdots=\beta_{M}$ (see [LeSp] for details).

Let us briefly discuss linear response theory in FGR thermodynamics using the same notational conventions as in Subsection 4.4. The kinetic coefficients are given by

$$
L_{\mathrm{fgr}, j i}=\partial_{X_{i}} \omega_{\mathcal{S}}+\left.\left(\Phi_{\mathrm{fgr}, j}\right)\right|_{X=0} .
$$

For $|X|<\epsilon$ one has

$$
\begin{gathered}
\omega_{\mathcal{S}+}\left(\Phi_{\mathrm{fgr}, j}\right)=\sum_{i=1}^{M} L_{\mathrm{fgr}, j i} X_{i}+O\left(\epsilon^{2}\right), \\
\operatorname{Ep}_{\mathrm{fgr}}\left(\omega_{\mathcal{S}+}\right)=\sum_{i, j=1}^{M} L_{\mathrm{fgr}, j i} X_{i} X_{j}+o\left(\epsilon^{2}\right) .
\end{gathered}
$$

The first and the second law yield that for all $i$,

$$
\sum_{j=1}^{M} L_{\mathrm{fgr}, j i}=0
$$

and that the quadratic form

$$
\sum_{i, j=1}^{M} L_{\mathrm{fgr}, j i} X_{i} X_{j}
$$

is non-negative. The Kubo formula

$$
L_{\mathrm{fgr}, j i}=\int_{0}^{\infty} \omega_{\beta_{\mathrm{eq}}}\left(\mathrm{e}^{t K_{\mathrm{H}}}\left(\Phi_{j}\right) \Phi_{i}\right) \mathrm{d} t,
$$


and the Onsager reciprocity relations

$$
L_{\mathrm{fgr}, j i}=L_{\mathrm{fgr}, i j},
$$

are proven in [LeSp].

Finally, we wish to comment on the relation between microscopic and FGR thermodynamics. One naturally expects FGR thermodynamics to produce the first non-trivial contribution (in $\lambda$ ) to the microscopic thermodynamics. For example, the following relations are expected to hold for small $\lambda$ :

$$
\begin{gathered}
\omega_{\lambda+}=\omega_{\mathcal{S}+}+O(\lambda), \\
\omega_{\lambda+}\left(\Phi_{j}\right)=\lambda^{2} \omega_{\mathcal{S}+}\left(\Phi_{\mathrm{fgr}, j}\right)+O\left(\lambda^{3}\right) .
\end{gathered}
$$

Indeed, it is possible to prove that if the microscopic thermodynamics exists and is sufficiently regular, then (4.28) hold. On the other hand, establishing existence and regularity of the microscopic thermodynamics is a formidable task which has been so far carried out only for a few models. FGR thermodynamics is very robust and the weak coupling limit is an effective tool in the study of the models whose microscopic thermodynamics appears beyond reach of the existing techniques.

We will return to this topic in Section 8 where we will discuss the FGR thermodynamics of the SEBB model.

\section{Free Fermi gas reservoir}

In the SEBB model, which we shall study in the second part of this lecture, the reservoir will be described by an infinitely extended free Fermi gas. Our description of the free Fermi gas in this section is suited to this application.

The basic properties of the free Fermi gas are discussed in the lecture [Me3] and in Examples 18 and 51 of the lecture [Pi] and we will assume that the reader is familiar with the terminology and results described there. A more detailed exposition can be found in [BR2] and in the recent lecture notes [D2].

The free Fermi gas is described by the so called CAR (canonical anticommutation relations) algebra. The mathematical structure of this algebra is well understood (see [D2] for example). In Subsection 5.1 we will review the results we need. Subsection 5.2 contains a few useful examples.

\subsection{General description}

Let $\mathfrak{h}$ and $h$ be the Hilbert space and the Hamiltonian of a single Fermion. We will always assume that $h$ is bounded below. Let $\Gamma_{-}(\mathfrak{h})$ be the anti-symmetric Fock space over $\mathfrak{h}$ and denote by $a^{*}(f), a(f)$ the creation and annihilation operators for a single Fermion in the state $f \in \mathfrak{h}$. The corresponding self-adjoint field operator

$$
\varphi(f) \equiv \frac{1}{\sqrt{2}}\left(a(f)+a^{*}(f)\right),
$$


satisfies the anticommutation relation

$$
\varphi(f) \varphi(g)+\varphi(g) \varphi(f)=\operatorname{Re}(f, g) I .
$$

In the sequel $a^{\#}$ stands for either $a$ or $a^{*}$. Let $\operatorname{CAR}(\mathfrak{h})$ be the $C^{*}$-algebra generated by $\left\{a^{\#}(f) \mid f \in \mathfrak{h}\right\}$. We will refer to $\operatorname{CAR}(\mathfrak{h})$ as the Fermi algebra. The $C^{*}$-dynamics induced by $h$ is

$$
\tau^{t}(A) \equiv \mathrm{e}^{\mathrm{i} t \mathrm{~d} \Gamma(h)} A \mathrm{e}^{-\mathrm{i} t \mathrm{~d} \Gamma(h)} .
$$

The pair $(\operatorname{CAR}(\mathfrak{h}), \tau)$ is a $C^{*}$-dynamical system. It preserves the Fermion number in the sense that $\tau^{t}$ commutes with the gauge group

$$
\vartheta^{t}(A) \equiv \mathrm{e}^{\mathrm{i} t \mathrm{~d} \Gamma(I)} A \mathrm{e}^{-\mathrm{i} t \mathrm{~d} \Gamma(I)} .
$$

Recall that $N \equiv \mathrm{d} \Gamma(I)$ is the Fermion number operator on $\Gamma_{-}(\mathfrak{h})$ and that $\tau$ and $\vartheta$ are the groups of Bogoliubov automorphisms

$$
\tau^{t}\left(a^{\#}(f)\right)=a^{\#}\left(\mathrm{e}^{\mathrm{i} t h} f\right), \quad \vartheta^{t}\left(a^{\#}(f)\right)=a^{\#}\left(\mathrm{e}^{\mathrm{i} t} f\right) .
$$

To every self-adjoint operator $T$ on $\mathfrak{h}$ such that $0 \leq T \leq I$ one can associate a state $\omega_{T}$ on $\operatorname{CAR}(\mathfrak{h})$ satisfying

$$
\omega_{T}\left(a^{*}\left(f_{n}\right) \cdots a^{*}\left(f_{1}\right) a\left(g_{1}\right) \cdots a\left(g_{m}\right)\right)=\delta_{n, m} \operatorname{det}\left\{\left(g_{i}, T f_{j}\right)\right\} .
$$

This $\vartheta$-invariant state is usually called the quasi-free gauge-invariant state generated by $T$. It is completely determined by its two point function

$$
\omega_{T}\left(a^{*}(f) a(g)\right)=(g, T f) .
$$

We will often call $T$ the density operator or simply the generator of the state $\omega_{T}$. Alternatively, quasi-free gauge-invariant states can be described by their action on the field operators. For any integer $n$ we define $\mathcal{P}_{n}$ as the set of all permutations $\pi$ of $\{1, \ldots, 2 n\}$ such that

$$
\pi(2 j-1)<\pi(2 j), \quad \text { and } \quad \pi(2 j-1)<\pi(2 j+1),
$$

for every $j \in\{1, \ldots, n\}$. Denote by $\epsilon(\pi)$ the signature of $\pi \in \mathcal{P}_{n} . \omega_{T}$ is the unique state on $\operatorname{CAR}(\mathfrak{h})$ with the following properties:

$$
\begin{aligned}
\omega_{T}\left(\varphi\left(f_{1}\right) \varphi\left(f_{2}\right)\right) & =\frac{1}{2}\left(f_{1}, f_{2}\right)-\mathrm{i} \operatorname{Im}\left(f_{1}, T f_{2}\right), \\
\omega_{T}\left(\varphi\left(f_{1}\right) \cdots \varphi\left(f_{2 n}\right)\right) & =\sum_{\pi \in \mathcal{P}_{n}} \epsilon(\pi) \prod_{j=1}^{n} \omega_{T}\left(\varphi\left(f_{\pi(2 j-1)}\right) \varphi\left(f_{\pi(2 j)}\right)\right), \\
\omega_{T}\left(\varphi\left(f_{1}\right) \cdots \varphi\left(f_{2 n+1}\right)\right) & =0 .
\end{aligned}
$$

If $\mathfrak{h}=\mathfrak{h}_{1} \oplus \mathfrak{h}_{2}$ and $T=T_{1} \oplus T_{2}$, then for $A \in \operatorname{CAR}\left(\mathfrak{h}_{1}\right)$ and $B \in \operatorname{CAR}\left(\mathfrak{h}_{2}\right)$ one has

$$
\omega_{T}(A B)=\omega_{T_{1}}(A) \omega_{T_{2}}(B)
$$


$\omega_{T}$ is a factor state. It is modular iff $\operatorname{Ker} T=\operatorname{Ker}(I-T)=\{0\}$. Two states $\omega_{T_{1}}$ and $\omega_{T_{2}}$ are quasi-equivalent iff the operators

$$
T_{1}^{1 / 2}-T_{2}^{1 / 2} \quad \text { and } \quad\left(I-T_{1}\right)^{1 / 2}-\left(I-T_{2}\right)^{1 / 2},
$$

are Hilbert-Schmidt; see [De, PoSt, Ri]. Assume that $\operatorname{Ker} T_{i}=\operatorname{Ker}\left(I-T_{i}\right)=\{0\}$. Then the states $\omega_{T_{1}}$ and $\omega_{T_{2}}$ are unitarily equivalent iff (5.31) holds.

If $T=F(h)$ for some function $F: \sigma(h) \rightarrow[0,1]$, then $\omega_{T}$ describes a free Fermi gas with energy density per unit volume $F(\varepsilon)$.

The state $\omega_{T}$ is $\tau$-invariant iff $T$ commutes with $\mathrm{e}^{\mathrm{i} t h}$ for all $t$. If the spectrum of $h$ is simple this means that $T=F(h)$ for some function $F: \sigma(h) \rightarrow[0,1]$.

For any $\beta, \mu \in \mathbb{R}$, the Fermi-Dirac distribution $\rho_{\beta \mu}(\varepsilon) \equiv\left(1+\mathrm{e}^{\beta(\varepsilon-\mu)}\right)^{-1}$ induces the unique $\beta$-KMS state on $\operatorname{CAR}(\mathfrak{h})$ for the dynamics $\tau^{t} \circ \vartheta^{-\mu t}$. This state, which we denote by $\omega_{\beta \mu}$, describes the free Fermi gas at thermal equilibrium in the grand canonical ensemble with inverse temperature $\beta$ and chemical potential $\mu$.

The GNS representation of $\operatorname{CAR}(\mathfrak{h})$ associated to $\omega_{T}$ can be explicitly computed as follows. Fix a complex conjugation $f \mapsto \bar{f}$ on $\mathfrak{h}$ and extend it to $\Gamma_{-}(\mathfrak{h})$. Denote by $\Omega$ the vacuum vector and $N$ the number operator in $\Gamma_{-}(\mathfrak{h})$. Set

$$
\begin{aligned}
\mathcal{H}_{\omega_{T}} & =\Gamma_{-}(\mathfrak{h}) \otimes \Gamma_{-}(\mathfrak{h}), \\
\Omega_{\omega_{T}} & =\Omega \otimes \Omega \\
\pi_{\omega_{T}}(a(f)) & =a\left((I-T)^{1 / 2} f\right) \otimes I+(-I)^{N} \otimes a^{*}\left(\bar{T}^{1 / 2} \bar{f}\right) .
\end{aligned}
$$

The triple $\left(\mathcal{H}_{\omega_{T}}, \pi_{\omega_{T}}, \Omega_{\omega_{T}}\right)$ is the GNS representation of the algebra CAR(h) associated to $\omega_{T}$. (This representation was constructed in $[\mathrm{AW}]$ and if often called ArakiWyss representation.) If $\omega_{T}$ is $\tau$-invariant, the corresponding $\omega_{T}$-Liouvillean is

$$
L=\mathrm{d} \Gamma(h) \otimes I-I \otimes \mathrm{d} \Gamma(\bar{h}) .
$$

If $h$ has purely (absolutely) continuous spectrum so does $L$, except for the simple eigenvalue 0 corresponding to the vector $\Omega_{\omega_{T}}$. On the other hand, 0 becomes a degenerate eigenvalue as soon as $h$ has some point spectrum. Thus (see the lecture notes [Pi]) the ergodic properties of $\tau$-invariant, gauge-invariant quasi-free states can be described in terms of the spectrum of $h$. The state $\omega_{T}$ is ergodic iff $h$ has no eigenvalues. If $h$ has purely absolutely continuous spectrum, then $\omega_{T}$ is mixing.

If $\omega_{T}$ is modular, then its modular operator is

$$
\log \Delta_{\omega_{T}}=\mathrm{d} \Gamma(s) \otimes I-I \otimes \mathrm{d} \Gamma(\bar{s}),
$$

where $s=\log T(I-T)^{-1}$. The corresponding modular conjugation is $J(\Phi \otimes \Psi)=$ $u \bar{\Psi} \otimes u \bar{\Phi}$, where $u=(-I)^{N(N+I) / 2}$.

Let $\theta$ be the $*$-automorphism of $\operatorname{CAR}(\mathfrak{h})$ defined by

$$
\theta(a(f))=-a(f) .
$$

$A \in \mathrm{CAR}(\mathfrak{h})$ is called even if $\theta(A)=A$ and odd if $\theta(A)=-A$. Every element $A \in \operatorname{CAR}(\mathfrak{h})$ can be written in a unique way as a sum $A=A^{+}+A^{-}$where $A^{ \pm}=$ 
$(A \pm \theta(A)) / 2$ is even/odd. The set of all even/odd elements is a vector subspace of $\operatorname{CAR}(\mathfrak{h})$ and $\operatorname{CAR}(\mathfrak{h})$ is a direct sum of these two subspaces. It follows from (5.29) that $\omega_{T}(A)=0$ if $A$ is odd. Therefore one has $\omega_{T}(A)=\omega_{T}\left(A^{+}\right)$and

$$
\omega_{T} \circ \theta=\omega_{T} .
$$

The subspace of even elements is a $C^{*}$-subalgebra of $\operatorname{CAR}(\mathfrak{h})$. This subalgebra is called even CAR algebra and is denoted by $\mathrm{CAR}^{+}(\mathfrak{h})$. It is generated by

$$
\left\{a^{\#}\left(f_{1}\right) \cdots a^{\#}\left(f_{2 n}\right) \mid n \in \mathbb{N}, f_{j} \in \mathfrak{h}\right\} .
$$

The even CAR algebra plays an important role in physics. It is preserved by $\tau$ and $\vartheta$ and the pair $\left(\mathrm{CAR}^{+}(\mathfrak{h}), \tau\right)$ is a $C^{*}$-dynamical system.

We denote the restriction of $\omega_{T}$ to $\mathrm{CAR}^{+}(\mathfrak{h})$ by the same letter. In particular, $\omega_{\beta \mu}$ is the unique $\beta$-KMS state on $\mathrm{CAR}^{+}(\mathfrak{h})$ for the dynamics $\tau^{t} \circ \vartheta^{-\mu t}$.

Let

$$
A=a^{\#}\left(f_{1}\right) \cdots a^{\#}\left(f_{n}\right), \quad B=a^{\#}\left(g_{1}\right) \cdots a^{\#}\left(g_{m}\right),
$$

be two elements of $\operatorname{CAR}(\mathfrak{h})$, where $m$ is even. It follows from CAR that

$$
\left\|\left[A, \tau^{t}(B)\right]\right\| \leq C \sum_{i, j}\left|\left(f_{i}, \mathrm{e}^{\mathrm{i} t h} g_{j}\right)\right|
$$

where one can take $C=\left(\max \left(\left\|f_{i}\right\|,\left\|g_{j}\right\|\right)\right)^{n+m-2}$. If the functions $\left|\left(f_{i}, \mathrm{e}^{\mathrm{i} t h} g_{j}\right)\right|$ belong to $L^{1}(\mathbb{R}, \mathrm{d} t)$, then

$$
\int_{-\infty}^{\infty}\left\|\left[A, \tau^{t}(B)\right]\right\| \mathrm{d} t<\infty .
$$

Let $\mathfrak{h}_{0} \subset \mathfrak{h}$ be a subspace such that for any $f, g \in \mathfrak{h}_{0}$ the function $t \mapsto\left(f, \mathrm{e}^{\mathrm{i} t h} g\right)$ is integrable. Let $\mathcal{O}_{0}=\left\{a^{\#}\left(f_{1}\right) \cdots a^{\#}\left(f_{n}\right) \mid n \in \mathbb{N}, f_{j} \in \mathfrak{h}_{0}\right\}$ and let $\mathcal{O}_{0}^{+}$be the even subalgebra of $\mathcal{O}_{0}$. Then for $A \in \mathcal{O}_{0}$ and $B \in \mathcal{O}_{0}^{+}$(5.34) holds. If $\mathfrak{h}_{0}$ is dense in $\mathfrak{h}$, then $\mathcal{O}_{0}$ is dense in $\operatorname{CAR}(\mathfrak{h})$ and $\mathcal{O}_{0}^{+}$is dense in $\mathrm{CAR}^{+}(\mathfrak{h})$.

Let $\mathfrak{h}_{1}$ and $\mathfrak{h}_{2}$ be two Hilbert spaces, and let $\Omega_{\mathfrak{h}_{1}}, \Omega_{\mathfrak{h}_{2}}$ be the vaccua in $\Gamma_{-}\left(\mathfrak{h}_{1}\right)$ and $\Gamma_{-}\left(\mathfrak{h}_{2}\right)$. The exponential law for Fermions (see [BSZ] and [BR2], Example 5.2.20) states that there exists a unique unitary map $U: \Gamma_{-}\left(\mathfrak{h}_{1} \oplus \mathfrak{h}_{2}\right) \rightarrow \Gamma_{-}\left(\mathfrak{h}_{1}\right) \otimes \Gamma_{-}\left(\mathfrak{h}_{2}\right)$ such that

$$
\begin{gathered}
U \Omega_{\mathfrak{h}_{1} \oplus \mathfrak{h}_{2}}=\Omega_{\mathfrak{h}_{1}} \otimes \Omega_{\mathfrak{h}_{2}}, \\
U a(f \oplus g) U^{-1}=a(f) \otimes I+(-I)^{N} \otimes a(g), \\
U a^{*}(f \oplus g) U^{-1}=a^{*}(f) \otimes I+(-I)^{N} \otimes a^{*}(g), \\
U \mathrm{~d} \Gamma\left(h_{1} \oplus h_{2}\right) U^{-1}=\mathrm{d} \Gamma\left(h_{1}\right) \otimes I+I \otimes \mathrm{d} \Gamma\left(h_{2}\right) .
\end{gathered}
$$

The presence of the factors $(-I)^{N}$ in the above formulas complicates the description of a system containing several reservoirs. The following discussion should help the reader to understand its physical origin. 
Consider two boxes $\mathcal{R}_{1}, \mathcal{R}_{2}$ with one particle Hilbert spaces $\mathfrak{h}_{i} \equiv L^{2}\left(\mathcal{R}_{i}\right)$. Denote by $\mathcal{R}$ the combined box i.e., the disjoint union of $\mathcal{R}_{1}$ and $\mathcal{R}_{2}$. The corresponding one particle Hilbert space is $\mathfrak{h} \equiv L^{2}(\mathcal{R})$. Identifying the wave function $\Psi_{1}$ of an electron in $\mathcal{R}_{1}$ with $\Psi_{1} \oplus 0$ and similarly for an electron in $\mathcal{R}_{2}$ we can replace $\mathfrak{h}$ with the direct $\operatorname{sum} \mathfrak{h}_{1} \oplus \mathfrak{h}_{2}$.
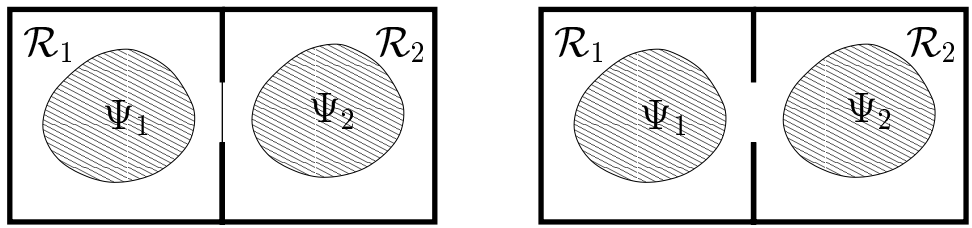

Figure 2: Thermal contact and open gate between $\mathcal{R}_{1}$ and $\mathcal{R}_{2}$.

Assume that each box $\mathcal{R}_{i}$ contains a single electron with wave functions $\Psi_{i}$ (see Fig. 2). If the boxes are in thermal contact, the two electrons can exchange energy, but the first one will always stay in $\mathcal{R}_{1}$ and the second one in $\mathcal{R}_{2}$. Thus they are distinguishable and the total wave function is just $\Psi_{1} \otimes \Psi_{2}$. The situation is completely different if the electrons are free to move from one box into the other. In this case, the electrons are indistinguishable and Pauli's principle requires the total wave function to be antisymmetric - the total wave function is $\Psi_{1} \wedge \Psi_{2}$. Generalizing this argument to many electrons states we conclude that the second quantized Hilbert space is $\Gamma_{-}\left(\mathfrak{h}_{1}\right) \otimes \Gamma_{-}\left(\mathfrak{h}_{2}\right)$ in the case of thermal contact and $\Gamma_{-}\left(\mathfrak{h}_{1} \oplus \mathfrak{h}_{2}\right)$ in the other case. The exponential law provides a unitary map $U$ between these two Hilbert and one easily checks that

$$
\begin{aligned}
U \Psi_{1} \wedge \Psi_{2} & =U a^{*}\left(\Psi_{1} \oplus 0\right) a^{*}\left(0 \oplus \Psi_{2}\right) \Omega_{\mathfrak{h}_{1} \oplus \mathfrak{h}_{2}} \\
& =\left(a^{*}\left(\Psi_{1}\right)(-I)^{N} \otimes a^{*}\left(\Psi_{2}\right)\right) \Omega_{\mathfrak{h}_{1}} \otimes \Omega_{\mathfrak{h}_{2}} \\
& =\Psi_{1} \otimes \Psi_{2} .
\end{aligned}
$$

Denoting by $\mathcal{O}_{\mathcal{R}_{1}}, \mathcal{O}_{\mathcal{R}_{2}}$ and $\mathcal{O}_{\mathcal{R}}$ the CAR (or more appropriately the $\mathrm{CAR}^{+}$) algebras of the boxes $\mathcal{R}_{1}, \mathcal{R}_{2}$ and $\mathcal{R}$, the algebra of the combined system in the case of thermal contact is $\mathcal{O}_{\mathcal{R}_{1}} \otimes \mathcal{O}_{\mathcal{R}_{2}}$, while it is $\mathcal{O}_{\mathcal{R}}$ in the other case. We emphasize that the unitary map $U$ does not yield an isomorphism between these algebras i.e.,

$$
U \mathcal{O}_{\mathcal{R}} U^{*} \neq \mathcal{O}_{\mathcal{R}_{1}} \otimes \mathcal{O}_{\mathcal{R}_{2}}
$$

This immediately follows from the observation that $(-I)^{N} \notin \mathcal{O}_{\mathcal{R}_{1}}$ (unless, of course, $\mathcal{O}_{\mathcal{R}_{1}}$ is finite dimensional, see Subsection 6.3), which implies

$$
U a^{*}\left(0 \oplus \Psi_{2}\right) U^{*}=(-I)^{N} \otimes a^{*}\left(\Psi_{2}\right) \notin \mathcal{O}_{\mathcal{R}_{1}} \otimes \mathcal{O}_{\mathcal{R}_{2}} .
$$

Note in particular that $a^{*}\left(\Psi_{1}\right) \otimes I$ and $I \otimes a^{*}\left(\Psi_{2}\right)$ commute while $a^{*}\left(\Psi_{1} \oplus 0\right)$ and $a^{*}\left(0 \oplus \Psi_{2}\right)$ anticommute. The factor $(-I)^{N}$ is required in order for $a^{*}\left(\Psi_{1}\right) \otimes I$ and $(-I)^{N} \otimes a^{*}\left(\Psi_{2}\right)$ to anticommute. 


\subsection{Examples}

Recall that the Pauli matrices are defined by

$$
\sigma_{x} \equiv\left[\begin{array}{cc}
0 & 1 \\
1 & 0
\end{array}\right], \quad \sigma_{y} \equiv\left[\begin{array}{cc}
0 & -\mathrm{i} \\
\mathrm{i} & 0
\end{array}\right], \quad \sigma_{z} \equiv\left[\begin{array}{cc}
1 & 0 \\
0 & -1
\end{array}\right] .
$$

We set $\sigma_{ \pm} \equiv\left(\sigma_{x} \pm \mathrm{i} \sigma_{y}\right) / 2$. Clearly, $\sigma_{x}^{2}=\sigma_{y}^{2}=\sigma_{z}^{2}=I$ and $\sigma_{x} \sigma_{y}=-\sigma_{y} \sigma_{x}=\mathrm{i} \sigma_{z}$. More generally, with $\vec{\sigma}=\left(\sigma_{x}, \sigma_{y}, \sigma_{z}\right)$ and $\vec{u}, \vec{v} \in \mathbb{R}^{3}$ one has

$$
(\vec{u} \cdot \vec{\sigma})(\vec{v} \cdot \vec{\sigma})=\vec{u} \cdot \vec{v} I+\mathrm{i}(\vec{u} \times \vec{v}) \cdot \vec{\sigma}
$$

Example 1. Assume that $\operatorname{dim} \mathfrak{h}=1$, i.e., that $\mathfrak{h}=\mathbb{C}$ and that $h$ is the operator of multiplication by the real constant $\omega$. Then $\Gamma_{-}(\mathfrak{h})=\mathbb{C} \oplus \mathbb{C}=\mathbb{C}^{2}$ and $\mathrm{d} \Gamma(h)=\omega N$ with

$$
N \equiv \mathrm{d} \Gamma(I)=\left[\begin{array}{ll}
0 & 0 \\
0 & 1
\end{array}\right]=\frac{1}{2}\left(I-\sigma_{z}\right) .
$$

Moreover, one easily checks that

$$
\begin{aligned}
& a(1)=\left[\begin{array}{ll}
0 & 0 \\
1 & 0
\end{array}\right], \quad a^{*}(1)=\left[\begin{array}{ll}
0 & 1 \\
0 & 0
\end{array}\right] \\
& a^{*}(1) a(1)=\left[\begin{array}{ll}
1 & 0 \\
0 & 0
\end{array}\right], \quad a(1) a^{*}(1)=\left[\begin{array}{ll}
0 & 0 \\
0 & 1
\end{array}\right] \text {, }
\end{aligned}
$$

which shows that $\mathrm{CAR}(\mathfrak{h})$ is the algebra of $2 \times 2$ matrices $M_{2}(\mathbb{C})$ and $\mathrm{CAR}^{+}(\mathfrak{h})$ its subalgebra of diagonal matrices. A self-adjoint operator $0 \leq T \leq I$ on $\mathcal{H}$ is multiplication by a constant $\gamma, 0 \leq \gamma \leq 1$. The associated state $\omega_{T}$ on $\operatorname{CAR}(\mathfrak{h})$ is given by the density matrix

$$
\left[\begin{array}{cc}
1-\gamma & 0 \\
0 & \gamma
\end{array}\right]
$$

Example 2. Assume that $\operatorname{dim} \mathfrak{h}=n$. Without loss of generality we can set $\mathfrak{h}=\mathbb{C}^{n}$ and assume that $h f_{j}=\omega_{j} f_{j}$ for some $\omega_{j} \in \mathbb{R}$, where $\left\{f_{j}\right\}$ is the standard basis of $\mathbb{C}^{n}$. Then,

$$
\Gamma_{-}(\mathfrak{h})=\mathbb{C}^{n} \oplus \mathbb{C}^{n} \wedge \mathbb{C}^{n} \oplus \cdots \oplus\left(\mathbb{C}^{n}\right)^{\wedge n} \simeq \bigotimes_{i=1}^{n} \mathbb{C}^{2},
$$

and $\operatorname{CAR}(\mathfrak{h})$ is isomorphic to the algebra of $2^{n} \times 2^{n}$ matrices $M_{2^{n}}(\mathbb{C})$. This isomorphism is explicitly given by

$$
a\left(f_{j}\right) \simeq\left(\otimes_{i=1}^{j-1} \sigma_{z}\right) \otimes \sigma_{+} \otimes\left(\otimes_{i=j+1}^{n} I\right)
$$

for $j=1, \ldots, n$. It follows that

$$
a^{*}\left(f_{j}\right) a\left(f_{j}\right) \simeq \frac{1}{2}\left(\otimes_{i=1}^{j-1} I\right) \otimes\left(I-\sigma_{z}\right) \otimes\left(\otimes_{i=j+1}^{n} I\right) .
$$


The map described by the above formulas is called the Jordan-Wigner transformation. It is a useful tool in the study of quantum spin systems (see [LMS, AB, Ar3]). For $\beta, \mu \in \mathbb{R}$, the quasi-free gauge-invariant state associated to $T=\left(I+\mathrm{e}^{\beta(h-\mu)}\right)^{-1}$ is given by the density matrix

$$
\frac{\mathrm{e}^{-\beta(H-\mu N)}}{\operatorname{Tr} \mathrm{e}^{-\beta(H-\mu N)}}
$$

with

$$
H \equiv \mathrm{d} \Gamma(h)=\sum_{j=1}^{n} \omega_{j} a^{*}\left(f_{j}\right) a\left(f_{j}\right), \quad N \equiv \mathrm{d} \Gamma(I)=\sum_{j=1}^{n} a^{*}\left(f_{j}\right) a\left(f_{j}\right) .
$$

It is an instructive exercise to work out the thermodynamics of the finite dimensional free Fermi gas following Section 3 in [Jo].

Example 3. In this example we will briefly discuss the finite dimensional approximation of a free Fermi gas. Assume that $\mathfrak{h}$ is a separable Hilbert space and let $\Lambda_{n} \subset$ Dom $h$ be an increasing sequence of finite dimensional subspaces. The algebras $\operatorname{CAR}\left(\Lambda_{n}\right)$ are identified with subalgebras of $\operatorname{CAR}(\mathfrak{h})$. We also assume that $\cup_{n} \Lambda_{n}$ is dense in $\mathfrak{h}$. Let $p_{n}$ be the orthogonal projection on $\Lambda_{n}$. Set $h_{n}=p_{n} h p_{n}$ and let $\tau_{n}$ be the corresponding $C^{*}$-dynamics on $\operatorname{CAR}\left(\Lambda_{n}\right)$. Since $p_{n}$ converges strongly to $I$ one has, for $f \in \mathcal{H}$,

$$
\lim _{n \rightarrow \infty}\left\|a^{\#}\left(p_{n} f\right)-a^{\#}(f)\right\|=0, \quad \lim _{n \rightarrow \infty}\left\|\tau_{n}^{t}\left(a^{\#}\left(p_{n} f\right)\right)-\tau^{t}\left(a^{\#}(f)\right)\right\|=0 .
$$

Let $\omega_{T}$ be the gauge-invariant quasi-free state on $\operatorname{CAR}(\mathfrak{h})$ associated to $T$. Let $T_{n}=$ $p_{n} T p_{n}$. Then

$$
\lim _{n \rightarrow \infty} \omega_{T_{n}}\left(a^{*}\left(p_{n} f\right) a\left(p_{n} g\right)\right)=\omega_{T}\left(a^{*}(f) a(g)\right) .
$$

Assume that $\mu$ and $\eta$ are two faithful $\omega_{T}$-normal states and let $\operatorname{Ent}(\mu \mid \eta)$ be their Araki relative entropy. Let $\mu_{n}$ and $\eta_{n}$ be the restrictions of $\mu$ and $\eta$ to $\operatorname{CAR}^{+}\left(\Lambda_{n}\right)$. Then the function

$$
n \mapsto \operatorname{Ent}\left(\mu_{n} \mid \eta_{n}\right)=\operatorname{Tr}_{\Lambda_{n}}\left(\mu_{n}\left(\log \mu_{n}-\log \eta_{n}\right)\right),
$$

is monotone increasing and

$$
\lim _{n \rightarrow \infty} \operatorname{Ent}\left(\mu_{n} \mid \eta_{n}\right)=\operatorname{Ent}(\mu \mid \eta)
$$

Additional information about the last result can be found in [BR2], Proposition 6.2.33.

Example 4. The tight binding approximation for an electron in a single Bloch band of a $d$-dimensional (cubic) crystal is defined by $\mathfrak{h} \equiv \ell^{2}\left(\mathbb{Z}^{d}\right)$ with the translation invariant Hamiltonian

$$
(h \psi)(x) \equiv \frac{1}{2 d} \sum_{|x-y|=1} \psi(y),
$$

where $|x| \equiv \sum_{i}\left|x_{i}\right|$. In the sequel $\delta_{x}$ denotes the Kronecker delta function at $x \in \mathbb{Z}^{d}$. 

by

Writing $a_{x} \equiv a\left(\delta_{x}\right)$, the second quantized energy and number operators are given

$$
\mathrm{d} \Gamma(h)=\frac{1}{2 d} \sum_{|x-y|=1} a_{x}^{*} a_{y}, \quad \mathrm{~d} \Gamma(I)=\sum_{x} a_{x}^{*} a_{x} .
$$

The Fourier transform $\hat{\psi}(k) \equiv \sum_{x} \psi(x) \mathrm{e}^{-i x \cdot k}$ maps $\mathfrak{h}$ unitarily onto

$$
\hat{\mathfrak{h}} \equiv L^{2}\left([-\pi, \pi]^{d}, \frac{\mathrm{d} k}{(2 \pi)^{d}}\right) .
$$

The set $[-\pi, \pi]^{d}$ is the Brillouin zone of the crystal and $k$ is the quasi-momentum of the electron. The Fourier transform diagonalizes the Hamiltonian which becomes multiplication by the band function $\varepsilon(k) \equiv \frac{1}{d} \sum_{i} \cos \left(k_{i}\right)$. Thus $h$ has purely absolutely continuous spectrum $\sigma(h)=[-1,1]$, and in particular is bounded.

A simple stationary phase argument shows that

$$
\left(f, \mathrm{e}^{\mathrm{i} t h} g\right)=\mathrm{O}\left(t^{-n}\right)
$$

for arbitrary $n$ provided $\hat{f}$ and $\hat{g}$ are smooth and vanish in a neighborhood of the critical set $\left\{k|| \nabla_{k} \varepsilon(k) \mid=0\right\}$. Since this set has Lebesgue measure 0 , such functions are dense in $\mathfrak{h}$. If $f$ and $g$ have bounded support in $\mathbb{Z}^{d}$, then

$$
\left(f, \mathrm{e}^{\mathrm{i} t h} g\right)=\mathrm{O}\left(t^{-d / 2}\right)
$$

Example 5. The tight binding approximation of a semi-infinite wire is obtained by restricting the Hamiltonian (5.37), for $d=1$, to the space of odd functions $\psi \in \ell^{2}(\mathbb{Z})$ and identifying such $\psi$ with elements of $\ell^{2}\left(\mathbb{Z}_{+}\right)$, where $\mathbb{Z}_{+} \equiv\{1,2, \cdots\}$. This is clearly equivalent to imposing a Dirichlet boundary condition at $x=0$ and

$$
h=\frac{1}{2} \sum_{x=1}^{\infty}\left(\left(\delta_{x}, \cdot\right) \delta_{x+1}+\left(\delta_{x+1}, \cdot\right) \delta_{x}\right) .
$$

The Fourier-sine transform $\tilde{\psi}(k) \equiv \sum_{x \in \mathbb{Z}_{+}} \psi(x) \sin (k x)$ maps unitarily $\ell^{2}\left(\mathbb{Z}_{+}\right)$onto the space $L^{2}\left([0, \pi], \frac{2 \mathrm{~d} k}{\pi}\right)$ and the Hamiltonian becomes multiplication by $\cos k$. By a simple change of variable $r=\cos k$ we obtain the spectral representation of the Hamiltonian $h$ :

$$
(h \psi)^{\#}(r)=r \psi^{\#}(r),
$$

where

$$
\psi^{\#}(r) \equiv \sqrt{\frac{2}{\pi \sqrt{1-r^{2}}}} \tilde{\psi}(\arccos (r)),
$$

maps unitarily the Fourier space $L^{2}\left([0, \pi], \frac{2 \mathrm{~d} k}{\pi}\right)$ onto $L^{2}([-1,1], \mathrm{d} r)$. A straightforward integration by parts shows that

$$
\left(f, \mathrm{e}^{\mathrm{i} t h} g\right)=\mathrm{O}\left(t^{-n}\right)
$$


if $f^{\#}, g^{\#} \in C_{0}^{n}((-1,1))$. A more careful analysis shows that

$$
\left(f, \mathrm{e}^{\mathrm{i} t h} g\right)=\mathrm{O}\left(t^{-3 / 2}\right),
$$

if $f$ and $g$ have bounded support in $\mathbb{Z}_{+}$.

Example 6. The non-relativistic spinless Fermion of mass $m$ is described in the position representation by the Hilbert space $L^{2}\left(\mathbb{R}^{d}, \mathrm{~d} x\right)$ and the Hamiltonian $h=-\Delta / 2 m$, where $\Delta$ is the usual Laplacian in $\mathbb{R}^{d}$. The cases of physical interest are $d=1,2,3$. In the momentum representation the Hilbert space of the Fermion is $L^{2}\left(\mathbb{R}^{d}, \mathrm{~d} k\right)$ and its Hamiltonian (which we will again denote by $h$ ) is the operator of multiplication by $|k|^{2} / 2 m$.

The spectrum of $h$ is purely absolutely continuous. Integration by parts yields that

$$
\left(f, \mathrm{e}^{\mathrm{i} t h} g\right)=\mathrm{O}\left(t^{-n}\right)
$$

for arbitrary $n$ provided $\hat{f}$ and $\hat{g}$ are smooth, compactly supported and vanish in a neighborhood of the origin. Such functions are dense in $\mathfrak{h}$. If $f, g \in \mathfrak{h}$ are compactly supported in the position representation, then

$$
\left(f, \mathrm{e}^{\mathrm{i} t h} g\right)=O\left(t^{-d / 2}\right) .
$$

\section{The simple electronic black-box (SEBB) model}

In the second part of this lecture we shall study in detail the non-equilibrium statistical mechanics of the simplest non-trivial example of the electronic black box model introduced in [AJPP]. The electronic black-box model is a general, independent electron model for a localized quantum device $\mathcal{S}$ connected to $M$ electronic reservoirs $\mathcal{R}_{1}, \cdots, \mathcal{R}_{M}$. The device is called black-box since, according to the scattering approach introduced in Subsection 4.2, the thermodynamics of the coupled system is largely independent of the internal structure of the device. The NESS and the steady currents are completely determined by the Møller morphism which in our simple model further reduces to the one-particle wave operator.

\subsection{The model}

The black-box itself is a two level system. Its Hilbert space is $\mathcal{H}_{\mathcal{S}} \equiv \mathbb{C}^{2}$, its algebra of observables is $\mathcal{O}_{\mathcal{S}} \equiv M_{2}(\mathbb{C})$, and its Hamiltonian is

$$
H_{\mathcal{S}} \equiv\left[\begin{array}{cc}
0 & 0 \\
0 & \varepsilon_{0}
\end{array}\right] \text {. }
$$

The associated $C^{*}$-dynamics is $\tau_{\mathcal{S}}^{t}(A)=\mathrm{e}^{\mathrm{i} t H_{\mathcal{S}}} A \mathrm{e}^{-\mathrm{i} t H_{\mathcal{S}}}$. The black-box has a oneparameter family of steady states with density matrices

$$
\omega_{\mathcal{S}} \equiv\left[\begin{array}{cc}
1-\gamma & 0 \\
0 & \gamma
\end{array}\right], \quad \gamma \in[0,1]
$$


which we shall use as the reference states.

According to Example 1 of Subsection 5.2, we can also think of $\mathcal{S}$ as a free Fermi gas over $\mathbb{C}$, namely $H_{\mathcal{S}}=\Gamma_{-}(\mathbb{C}), H_{\mathcal{S}}=\mathrm{d} \Gamma\left(\varepsilon_{0}\right)=\varepsilon_{0} a^{*}(1) a(1)$ and $\mathcal{O}_{\mathcal{S}}=\mathrm{CAR}(\mathbb{C})$. In this picture, the black-box $\mathcal{S}$ can only accommodate a single Fermion of energy $\varepsilon_{0}$. We denote by $N_{\mathcal{S}}=a^{*}(1) a(1)$ the corresponding number operator. In physical terms, $\mathcal{S}$ is a quantum dot without internal structure. We also note that $\omega_{\mathcal{S}}$ is the quasifree gauge-invariant state generated by $T_{\mathcal{S}} \equiv \gamma$. Therefore, we can interpret $\gamma$ as the occupation probability of the box.

Let $\mathfrak{h}_{\mathcal{R}}$ be a Hilbert space and $h_{\mathcal{R}}$ a self-adjoint operator on $\mathfrak{h}_{\mathcal{R}}$. We set $\mathcal{O}_{\mathcal{R}} \equiv$ $\operatorname{CAR}\left(\mathfrak{h}_{\mathcal{R}}\right)$ and

$$
\tau_{\mathcal{R}}^{t}(A) \equiv \mathrm{e}^{\mathrm{i} t \mathrm{~d} \Gamma\left(h_{\mathcal{R}}\right)} A \mathrm{e}^{-\mathrm{i} t \mathrm{~d} \Gamma\left(h_{\mathcal{R}}\right)} .
$$

The reference state of the reservoir, $\omega_{\mathcal{R}}$, is the quasi-free gauge-invariant state associated to the radiation density operator $T_{\mathcal{R}}$. We assume that $h_{\mathcal{R}}$ is bounded from below and that $T_{\mathcal{R}}$ commutes with $h_{\mathcal{R}}$.

To introduce the subreservoir structure we shall assume that

$$
\mathfrak{h}_{\mathcal{R}}=\oplus_{j=1}^{M} \mathfrak{h}_{\mathcal{R}_{j}}, \quad h_{\mathcal{R}}=\oplus_{j=1}^{M} h_{\mathcal{R}_{j}}, \quad T_{\mathcal{R}}=\oplus_{j=1}^{M} T_{\mathcal{R}_{j}} .
$$

The algebra of observables of the $j$-th reservoir is $\mathcal{O}_{\mathcal{R}_{j}} \equiv \operatorname{CAR}\left(\mathfrak{h}_{\mathcal{R}_{j}}\right)$ and its dynamics $\tau_{\mathcal{R}_{j}} \equiv \tau_{\mathcal{R}}\left\lceil\mathcal{O}_{\mathcal{R}_{j}}\right.$ is generated by the Hamiltonian $\mathrm{d} \Gamma\left(h_{\mathcal{R}_{j}}\right)$. The state $\omega_{\mathcal{R}_{j}}=\omega_{\mathcal{R}} \uparrow$ $\mathcal{O}_{\mathcal{R}_{j}}$ is the gauge-invariant quasi-free state associated to $T_{\mathcal{R}_{j}}$. If $p_{j}$ is the orthogonal projection on $\mathfrak{h}_{\mathcal{R}_{j}}$, then $N_{\mathcal{R}_{j}}=\mathrm{d} \Gamma\left(p_{j}\right)$ is the charge (or number) operator associated to the $j$-th reservoir. The total charge operator of the reservoir is $N_{\mathcal{R}}=\sum_{j=1}^{M} N_{\mathcal{R}_{j}}$.

The algebra of observables of the joint system $\mathcal{S}+\mathcal{R}$ is $\mathcal{O} \equiv \mathcal{O}_{\mathcal{S}} \otimes \mathcal{O}_{\mathcal{R}}$, its reference state is $\omega=\omega_{\mathcal{S}} \otimes \omega_{\mathcal{R}}$, and its decoupled dynamics is $\tau_{0}=\tau_{\mathcal{S}} \otimes \tau_{\mathcal{R}}$. Note that

$$
\tau_{0}^{t}(A)=\mathrm{e}^{\mathrm{i} t H_{0}} A \mathrm{e}^{-\mathrm{i} t H_{0}},
$$

where

$$
H_{0} \equiv H_{\mathcal{S}} \otimes I+I \otimes \mathrm{d} \Gamma\left(h_{\mathcal{R}}\right) .
$$

The junction between the box $\mathcal{S}$ and the reservoir $\mathcal{R}_{j}$ works in the following way: The box can make a transition from its ground state to its excited state by absorbing an electron of $\mathcal{R}_{j}$ in state $f_{j} /\left\|f_{j}\right\|$. Reciprocally, the excited box can relax to its ground state by emitting an electron in state $f_{j} /\left\|f_{j}\right\|$ in $\mathcal{R}_{j}$. These processes have a fixed rate $\lambda^{2}\left\|f_{j}\right\|^{2}$. More precisely, the junction is described by

$$
\lambda V_{j} \equiv \lambda\left(a(1) \otimes a^{*}\left(f_{j}\right)+a^{*}(1) \otimes a\left(f_{j}\right)\right),
$$

where $\lambda \in \mathbb{R}$ and the $f_{j} \in \mathfrak{h}_{j}$. The normalization is fixed by the condition $\sum_{j}\left\|f_{j}\right\|^{2}=$ 1. The complete interaction is given by

$$
\lambda V \equiv \sum_{j=1}^{M} \lambda V_{j}=\lambda\left(a(1) \otimes a^{*}(f)+a^{*}(1) \otimes a(f)\right),
$$

where $f \equiv \oplus_{j=1}^{M} f_{j}$. Note that "charge" is conserved at the junction, i.e., $V$ commutes with the total number operator $N \equiv N_{\mathcal{S}} \otimes I+I \otimes N_{\mathcal{R}}$. 
The full Hamiltonian is

$$
H_{\lambda} \equiv H_{0}+\lambda V
$$

and the corresponding $C^{*}$-dynamics

$$
\tau_{\lambda}^{t}(A) \equiv \mathrm{e}^{\mathrm{i} t H_{\lambda}} A \mathrm{e}^{-\mathrm{i} t H_{\lambda}}
$$

is charge-preserving. In other words, $\tau_{\lambda}$ commutes with the gauge group

$$
\vartheta^{t}(A) \equiv \mathrm{e}^{\mathrm{i} t N} A \mathrm{e}^{-\mathrm{i} t N}
$$

and $\left[H_{\lambda}, N\right]=0$. The $C^{*}$-dynamical system $\left(\mathcal{O}, \tau_{\lambda}\right)$ with its decoupled dynamics $\tau_{0}^{t}$ and the reference state $\omega=\omega_{\mathcal{S}} \otimes \omega_{\mathcal{R}}$ is our simple electronic black box model (SEBB). This model is an example of the class of open quantum systems described in Section 4.

\subsection{The fluxes}

The heat flux observables have been defined in Subsection 4.3. The generator of $\tau_{\mathcal{R}_{j}}$ is given by $\delta_{j}(\cdot)=\mathrm{i}\left[\mathrm{d} \Gamma\left(h_{\mathcal{R}_{j}}\right), \cdot\right]$. Note that $V_{j} \in \operatorname{Dom} \delta_{j}$ iff $f_{j} \in \operatorname{Dom} h_{\mathcal{R}_{j}}$. If $V_{j} \in \operatorname{Dom} \delta_{j}$, then the observable describing the heat flux out of $\mathcal{R}_{j}$ is

$$
\Phi_{j}=\lambda \delta_{j}\left(V_{j}\right)=\lambda\left(a(1) \otimes a^{*}\left(\mathrm{i} h_{\mathcal{R}_{j}} f_{j}\right)+a^{*}(1) \otimes a\left(\mathrm{i} h_{\mathcal{R}_{j}} f_{j}\right)\right) .
$$

In a completely similar way we can define the charge current. The rate of change of the charge in the box $\mathcal{S}$ is

$$
\begin{aligned}
\left.\frac{\mathrm{d}}{\mathrm{d} t} \tau_{\lambda}^{t}\left(N_{\mathcal{S}}\right)\right|_{t=0} & =\mathrm{i}\left[\mathrm{d} \Gamma\left(H_{\lambda}\right), N_{\mathcal{S}}\right] \\
& =-\lambda \mathrm{i}\left[N_{\mathcal{S}}, V\right]=\lambda \mathrm{i}\left[N_{\mathcal{R}}, V\right]=\sum_{j=1}^{M} \lambda \mathrm{i}\left[N_{\mathcal{R}_{j}}, V\right],
\end{aligned}
$$

which allows us to identify

$$
\begin{aligned}
\mathcal{J}_{j} & \equiv \lambda \mathrm{i}\left[N_{\mathcal{R}_{j}}, V\right] \\
& =\lambda \mathrm{i}\left[N_{\mathcal{R}_{j}}, V_{j}\right]=\lambda \mathrm{i}\left[N_{\mathcal{R}}, V_{j}\right]=\lambda\left(a(1) \otimes a^{*}\left(\mathrm{i} f_{j}\right)+a^{*}(1) \otimes a\left(\mathrm{i} f_{j}\right),\right.
\end{aligned}
$$

as the observable describing the charge current out of $\mathcal{R}_{j}$.

Let us make a brief comment concerning these definitions. If $\mathfrak{h}_{\mathcal{R}_{j}}$ is finite dimensional, then the energy and the charge of $\mathcal{R}_{j}$ are observables, given by the Hamiltonian $\mathrm{d} \Gamma\left(h_{\mathcal{R}_{j}}\right)$ and the number operator $N_{\mathcal{R}_{j}}=\mathrm{d} \Gamma\left(p_{j}\right)$, and

$$
\begin{aligned}
-\left.\frac{\mathrm{d}}{\mathrm{d} t} \tau_{\lambda}^{t}\left(\mathrm{~d} \Gamma\left(h_{\mathcal{R}_{j}}\right)\right)\right|_{t=0} & =\lambda \mathrm{i}\left[\mathrm{d} \Gamma\left(h_{\mathcal{R}_{j}}\right), V_{j}\right]=\Phi_{j} \\
-\left.\frac{\mathrm{d}}{\mathrm{d} t} \tau_{\lambda}^{t}\left(\mathrm{~d} \Gamma\left(p_{j}\right)\right)\right|_{t=0} & =\lambda \mathrm{i}\left[\mathrm{d} \Gamma\left(p_{j}\right), V_{j}\right]=\mathcal{J}_{j} .
\end{aligned}
$$

When $\mathfrak{h}_{\mathcal{R}_{j}}$ becomes infinite dimensional (recall Example 3 in Subsection 5.2), $N_{\mathcal{R}_{j}}$ and $\mathrm{d} \Gamma\left(h_{\mathcal{R}_{j}}\right)$ are no longer observables. However, the flux observables $\Phi_{j}$ and $\mathcal{J}_{j}$ are 
still well-defined and they are equal to the limit of the flux observables corresponding to finite-dimensional approximations.

The first law of thermodynamics (energy conservation) has been verified in Subsection 4.3-for any $\tau_{\lambda}$-invariant state $\eta$ one has

$$
\sum_{j=1}^{M} \eta\left(\Phi_{j}\right)=0
$$

The analogous statement for charge currents is proved in a similar way. By (6.38),

$$
\sum_{j=1}^{M} \mathcal{J}_{j}=\left.\frac{\mathrm{d}}{\mathrm{d} t} \tau_{\lambda}^{t}\left(N_{\mathcal{S}}\right)\right|_{t=0}
$$

and so for any $\tau_{\lambda}$-invariant state $\eta$ one has

$$
\sum_{j=1}^{M} \eta\left(\mathcal{J}_{j}\right)=0
$$

\subsection{The equivalent free Fermi gas}

In this subsection we shall show how to use the exponential law for fermionic systems to map the SEBB model to a free Fermi gas. Let

$$
\mathfrak{h} \equiv \mathbb{C} \oplus \mathfrak{h}_{\mathcal{R}}=\mathbb{C} \oplus\left(\bigoplus_{j=1}^{M} \mathfrak{h}_{\mathcal{R}_{j}}\right), \quad \tilde{\mathcal{O}} \equiv \operatorname{CAR}(\mathfrak{h}), \quad h_{0} \equiv \varepsilon_{0} \oplus h_{\mathcal{R}}
$$

and, with a slight abuse of notation, denote by $1, f_{1}, \cdots, f_{M}$ the elements of $\mathfrak{h}$ canonically associated with $1 \in \mathbb{C}$ and $f_{j} \in \mathfrak{h}_{\mathcal{R}_{j}}$. Then

$$
v_{j} \equiv(1, \cdot) f_{j}+\left(f_{j}, \cdot\right) 1,
$$

is a finite rank, self-adjoint operator on $\mathfrak{h}$ and so is the sum $v \equiv \sum_{j=1}^{M} v_{j}$. We further set

$$
h_{\lambda} \equiv h_{0}+\lambda v,
$$

and define the dynamical group

$$
\tilde{\tau}_{\lambda}^{t}(A) \equiv \mathrm{e}^{\mathrm{i} t \mathrm{~d} \Gamma\left(h_{\lambda}\right)} A \mathrm{e}^{-\mathrm{i} t \mathrm{~d} \Gamma\left(h_{\lambda}\right)},
$$

on $\tilde{\mathcal{O}}$. Finally, we set

$$
\tilde{T} \equiv T_{\mathcal{S}} \oplus T_{\mathcal{R}},
$$

and denote by $\tilde{\omega}$ be the quasi-free gauge-invariant state on $\tilde{\mathcal{O}}$ generated by $\tilde{T}$.

Theorem 6.1 Let $U: \Gamma_{-}\left(\mathbb{C} \oplus \mathfrak{h}_{\mathcal{R}}\right) \rightarrow \Gamma_{-}(\mathbb{C}) \otimes \Gamma_{-}\left(\mathfrak{h}_{\mathcal{R}}\right)$ be the unitary map defined by the exponential law (5.35) and set $\phi(A) \equiv U^{-1} A U$. 
(i) $\phi: \mathcal{O} \rightarrow \tilde{\mathcal{O}}$ is a *-isomorphism.

(ii) For any $\lambda, t \in \mathbb{R}$ one has $\phi \circ \tau_{\lambda}^{t}=\tilde{\tau}_{-\lambda}^{t} \circ \phi$.

(iii) $\omega=\tilde{\omega} \circ \phi$.

(iv) For $j=1, \cdots, M$, one has

$$
\tilde{\Phi}_{j} \equiv \phi\left(\Phi_{j}\right)=-\lambda\left(a^{*}\left(\mathrm{i} h_{j} f_{j}\right) a(1)+a^{*}(1) a\left(\mathrm{i} h_{j} f_{j}\right)\right),
$$

and

$$
\tilde{\mathcal{J}}_{j} \equiv \phi\left(\mathcal{J}_{j}\right)=-\lambda\left(a^{*}\left(\mathrm{i} f_{j}\right) a(1)+a^{*}(1) a\left(\mathrm{i} f_{j}\right)\right) .
$$

Proof. Clearly, $\phi$ is a $*$-isomorphism from $\mathcal{B}\left(\Gamma_{-}(\mathbb{C} \oplus \mathfrak{h})\right)$ onto $\mathcal{B}\left(\Gamma_{-}(\mathbb{C}) \otimes \Gamma_{-}(\mathfrak{h})\right)$. Using the canonical injections $\mathbb{C} \rightarrow \mathfrak{h}$ and $\mathfrak{h}_{\mathcal{R}} \rightarrow \mathfrak{h}$ we can identify $\mathcal{O}_{\mathcal{S}}$ and $\mathcal{O}_{\mathcal{R}}$ with the subalgebras of $\tilde{\mathcal{O}}$ generated by $a(1 \oplus 0)$ and $\left\{a(0 \oplus f) \mid f \in \mathfrak{h}_{\mathcal{R}}\right\}$. With this identification, (5.35) gives

$$
\phi\left(a(\alpha) \otimes I+(-I)^{N_{\mathcal{S}}} \otimes a(f)\right)=a(\alpha)+a(f),
$$

for $\alpha \in \mathbb{C}$ and $f \in \mathfrak{h}_{\mathcal{R}}$. We conclude that

$$
\phi(A \otimes I)=A,
$$

for any $A \in \mathcal{O}_{\mathcal{S}}$. In particular, since $b \equiv(-I)^{N_{\mathcal{S}}}=\left[a(1), a^{*}(1)\right] \in \mathcal{O}_{\mathcal{S}}$, we have $\phi(b \otimes I)=b$. Relation $b^{2}=I$ yields $\phi(I \otimes a(f))=b a(f)$. Since $[b, a(f)]=0$, we conclude that for $A \in \mathcal{O}_{\mathcal{R}}$

$$
\phi(I \otimes A)= \begin{cases}A & \text { if } A \in \mathcal{O}_{\mathcal{R}}^{+}, \\ b A & \text { if } A \in \mathcal{O}_{\mathcal{R}}^{-},\end{cases}
$$

where $\mathcal{O}_{\mathcal{R}}^{ \pm}$denote the even and odd parts of $\mathcal{O}_{\mathcal{R}}$. Equ. (6.41) and (6.42) show that $\phi(\mathcal{O}) \subset \tilde{\mathcal{O}}$. Since $\tilde{\mathcal{O}}=\left\langle\mathcal{O}_{\mathcal{S}}, \mathcal{O}_{\mathcal{R}}^{+}, \mathcal{O}_{\mathcal{R}}^{-}\right\rangle$, it follows from $\phi\left(\mathcal{O}_{\mathcal{S}} \otimes I\right)=\mathcal{O}_{\mathcal{S}}, \phi(I \otimes$ $\left.\mathcal{O}_{\mathcal{R}}^{+}\right)=\mathcal{O}_{\mathcal{R}}^{+}$and $\phi\left(b \otimes \mathcal{O}_{\mathcal{R}}^{-}\right)=\mathcal{O}_{\mathcal{R}}^{-}$that $\phi(\mathcal{O}) \supset \tilde{\mathcal{O}}$. This proves Part (i).

From (5.35) we can see that $U^{-1} H_{0} U=\mathrm{d} \Gamma\left(h_{0}\right)$ and from (6.41) and (6.42) that

$$
U^{-1} V_{j} U=\phi\left(V_{j}\right)=a(1) b a^{*}\left(f_{j}\right)+a^{*}(1) b a\left(f_{j}\right) .
$$

Since it also follows from CAR that

$$
a(1) b=-a(1), \quad a^{*}(1) b=a^{*}(1),
$$

we get

$$
U^{-1} V_{j} U=-a(1) a^{*}\left(f_{j}\right)+a^{*}(1) a\left(f_{j}\right)=-a(1) a^{*}\left(f_{j}\right)-a\left(f_{j}\right) a^{*}(1)=-\mathrm{d} \Gamma\left(v_{j}\right) .
$$

Therefore $U^{-1} H_{\lambda} U=\mathrm{d} \Gamma\left(h_{-\lambda}\right)$ from which Part (ii) follows. A similar computation yields Part (iv). 
It remains to prove Part (iii). Using the morphism $\theta$ (recall Equ. (5.32)) to express the even and odd parts of $B \in \mathcal{O}_{\mathcal{R}}$, we can rewrite (6.41) and (6.42) as

$$
\phi(A \otimes B)=A(B+\theta(B)) / 2+A b(B-\theta(B)) / 2
$$

from which we easily get

$$
\phi(A \otimes B)=A a(1) a^{*}(1) B+A a^{*}(1) a(1) \theta(B) .
$$

It follows from the factorization property (5.30) and the invariance property (5.33) of quasi-free states that

$$
\begin{aligned}
\tilde{\omega} \circ \phi(A \otimes B) & =\tilde{\omega}\left(A a(1) a^{*}(1)\right) \tilde{\omega}(B)+\tilde{\omega}\left(A a^{*}(1) a(1)\right) \tilde{\omega}(B) \\
& =\tilde{\omega}\left(A a(1) a^{*}(1) B+A a^{*}(1) a(1) B\right) \\
& =\tilde{\omega}(A B)=\tilde{\omega}(A) \tilde{\omega}(B) \\
& =\omega_{\mathcal{S}}(A) \omega_{\mathcal{R}}(B)=\omega(A \otimes B)
\end{aligned}
$$

By Theorem 6.1, the SEBB model can be equivalently described by the $C^{*}$-dynamical system $\left(\tilde{O}, \tilde{\tau}_{-\lambda}\right)$ and the reference state $\omega_{\tilde{T}}$. The heat and charge flux observables are $\tilde{\Phi}_{j}$ and $\tilde{\mathcal{J}}_{j}$. Since the change $\lambda \rightarrow-\lambda$ affects neither the model nor the results, in the sequel we will work with the system $\left(\tilde{\mathcal{O}}, \tilde{\tau}_{\lambda}\right)$ and we will drop the $\sim$. Hence, we will use the $C^{*}$-algebra $\mathcal{O}=\operatorname{CAR}\left(\mathbb{C} \oplus \mathfrak{h}_{\mathcal{R}}\right)$ and $C^{*}$-dynamics

$$
\tau_{\lambda}^{t}(A)=\mathrm{e}^{\mathrm{i} t \mathrm{~d} \Gamma\left(h_{\lambda}\right)} A \mathrm{e}^{-\mathrm{i} t \mathrm{~d} \Gamma\left(h_{\lambda}\right)}
$$

with the reference state $\omega$, the quasi-free gauge-invariant state generated by $T=T_{\mathcal{S}} \oplus$ $T_{\mathcal{R}}$. The corresponding heat and charge flux observables are

$$
\begin{array}{r}
\Phi_{j} \equiv \lambda\left(a^{*}\left(\mathrm{i} h_{j} f_{j}\right) a(1)+a^{*}(1) a\left(\mathrm{i} h_{j} f_{j}\right)\right) \\
\mathcal{J}_{j} \equiv \lambda\left(a^{*}\left(\mathrm{i} f_{j}\right) a(1)+a^{*}(1) a\left(\mathrm{i} f_{j}\right)\right) .
\end{array}
$$

The entropy production observable associated to $\omega$ is computed as follows. Assume that for $j=1, \cdots, M$ one has $\operatorname{Ker} T_{\mathcal{R}_{j}}=\operatorname{Ker}\left(I-T_{\mathcal{R}_{j}}\right)=\{0\}$ and set

$$
s_{j} \equiv-\log T_{\mathcal{R}_{j}}\left(I-T_{\mathcal{R}_{j}}\right)^{-1}, \quad s_{\mathcal{R}}=\oplus_{j=1}^{M} s_{j} .
$$

We also assume that $0<\gamma<1$ and set $s_{\mathcal{S}}=\log \gamma(1-\gamma)^{-1}$. Let $s \equiv-s_{\mathcal{S}} \oplus s_{\mathcal{R}}$. Under the above assumptions, the reference state $\omega$ is modular and its modular automorphism group is

$$
\sigma_{\omega}^{t}(A)=\mathrm{e}^{\mathrm{i} t \mathrm{~d} \Gamma(s)} A \mathrm{e}^{-\mathrm{i} t \mathrm{~d} \Gamma(s)}
$$

If $f_{j} \in \operatorname{Dom}\left(s_{j}\right)$, then the entropy production observable is

$$
\sigma=-\lambda\left(a^{*}(f) a\left(\mathrm{i} s_{\mathcal{S}}\right)+a^{*}\left(\mathrm{i} s_{\mathcal{S}}\right) a(f)\right)-\lambda\left(a^{*}\left(\mathrm{i} s_{\mathcal{R}} f\right) a(1)+a^{*}(1) a\left(\mathrm{i} s_{\mathcal{R}} f\right)\right) .
$$


The entropy balance equation

$$
\operatorname{Ent}\left(\omega \circ \tau_{\lambda}^{t} \mid \omega\right)=-\int_{0}^{t} \omega\left(\tau_{\lambda}^{s}(\sigma)\right) \mathrm{d} s
$$

holds and so, as in Subsection 3.2, the entropy production of any NESS $\omega_{+} \in \Sigma_{+}\left(\omega, \tau_{\lambda}\right)$ is non-negative. In fact, it is not difficult to show that the entropy production of $\omega_{+}$is independent of $\gamma$ as long as $\gamma \in(0,1)$ (see Proposition 5.3 in [JP4]). In the sequel, whenever we speak about the entropy production, we will assume that $\gamma=1 / 2$ and hence that

$$
\sigma=-\lambda\left(a^{*}\left(\mathrm{i} s_{\mathcal{R}} f\right) a(1)+a^{*}(1) a\left(\mathrm{i} s_{\mathcal{R}} f\right)\right)
$$

In particular, if

$$
T_{\mathcal{R}_{j}}=\left(I+\mathrm{e}^{\beta_{j}\left(h_{\mathcal{R}_{j}}-\mu_{j}\right)}\right),
$$

then $s_{j}=-\beta_{j}\left(h_{\mathcal{R}_{j}}-\mu_{j}\right)$, and

$$
\sigma=-\sum_{j=1}^{M} \beta_{j}\left(\Phi_{j}-\mu_{j} \mathcal{J}_{j}\right)
$$

We finish with the following remark. In the physics literature, the Hamiltonian (6.40) is sometimes called the Wigner-Weisskopf atom [WW] (see [JKP] for references and additional information). The operators of this type are also often called Friedrich Hamiltonians [Fr]. The point we wish to emphasize is that such Hamiltonians are often used as toy models which allow for simple mathematical analysis of physically important phenomena.

\subsection{Assumptions}

In this subsection we describe a set of assumptions under which we shall study the thermodynamics of the SEBB model.

Assumption (SEBB1) $\mathfrak{h}_{\mathcal{R}_{j}}=L^{2}\left(\left(e_{-}, e_{+}\right), \mathrm{d} r\right)$ for some $-\infty<e_{-}<e_{+} \leq \infty$ and $h_{\mathcal{R}_{j}}$ is the operator of multiplication by $r$.

The assumption (SEBB1) yields that $\mathfrak{h}_{\mathcal{R}}=L^{2}\left(\left(e_{-}, e_{+}\right), \mathrm{d} r ; \mathbb{C}^{M}\right)$ and that $h_{\mathcal{R}}$ is the operator of multiplication by $r$. With a slight abuse of the notation we will sometimes denote $h_{\mathcal{R}_{j}}$ and $h_{\mathcal{R}}$ by $r$. Note that the spectrum of $h_{\mathcal{R}}$ is purely absolutely continuous and equal to $\left[e_{-}, e_{+}\right]$with uniform multiplicity $M$. With the shorthand $f \equiv\left(f_{1}, \cdots, f_{M}\right) \in \mathfrak{h}_{\mathcal{R}}$, the Hamiltonian (6.40) acts on $\mathbb{C} \oplus \mathfrak{h}_{\mathcal{R}}$ and has the form

$$
h_{\lambda}=\varepsilon_{0} \oplus r+\lambda((1, \cdot) f+(f, \cdot) 1) .
$$

Assumption (SEBB2) The functions

$$
g_{j}(t) \equiv \int_{e_{-}}^{e_{+}} \mathrm{e}^{\mathrm{i} t r}\left|f_{j}(r)\right|^{2} \mathrm{~d} r
$$


belong to $L^{1}(\mathbb{R}, \mathrm{d} t)$.

Assumption (SEBB2) implies that the function

$$
G(z) \equiv \int_{e_{-}}^{e_{+}} \frac{|f(r)|^{2}}{r-z} \mathrm{~d} r=-\mathrm{i} \int_{0}^{\infty} g(t) \mathrm{e}^{-\mathrm{i} t z} \mathrm{~d} t
$$

which is obviously analytic in the lower half-plane $\mathbb{C}_{-} \equiv\{z \mid \operatorname{Im} z<0\}$, is continuous and bounded on its closure $\overline{\mathbb{C}}_{-}$. We denote by $G(r-\mathrm{i} o)$ the value of this function at $r \in \mathbb{R}$.

Assumption (SEBB3) For $j=1, \cdots, M$, the generator $T_{\mathcal{R}_{j}}$ is the operator of multiplication by a continuous function $\rho_{j}(r)$ such that $0<\rho_{j}(r)<1$ for $r \in\left(e_{-}, e_{+}\right)$. Moreover, if

$$
s_{j}(r) \equiv \log \left[\frac{\rho_{j}(r)}{1-\rho_{j}(r)}\right]
$$

we assume that $s_{j}(r) f_{j}(r) \in L^{2}\left(\left(e_{-}, e_{+}\right), \mathrm{d} r\right)$.

Assumption (SEBB3) ensures that the reference state $\omega_{\mathcal{R}}$ of the reservoir is modular. The function $\rho_{j}(r)$ is the energy density of the $j$-th reservoir. The second part of this assumption ensures that the entropy production observable (6.44) is well defined.

The study of SEBB model depends critically on the spectral and scattering properties of $h_{\lambda}$. Our final assumption will ensure that Assumption (S) of Subsection 3.4 holds and will allow us to use a simple scattering approach to study SEBB.

Assumption (SEBB4) $\varepsilon_{0} \in\left(e_{-}, e_{+}\right)$and $\left|f\left(\varepsilon_{0}\right)\right| \neq 0$.

We set

$$
F(r) \equiv \varepsilon_{0}-r-\lambda^{2} G(r-\mathrm{io})=\varepsilon_{0}-r-\lambda^{2} \int_{e_{-}}^{e_{+}} \frac{\left|f\left(r^{\prime}\right)\right|^{2}}{r^{\prime}-r+\mathrm{io}} \mathrm{d} r^{\prime} .
$$

By a well-known result in harmonic analysis (see, e.g., [Ja] or any harmonic analysis textbook),

$$
\operatorname{Im} F(r)=\lambda^{2} \pi|f(r)|^{2},
$$

for $r \in\left(e_{-}, e_{+}\right)$. We also mention that for any $g \in \mathfrak{h}_{\mathcal{R}}=L^{2}\left(\left(e_{-}, e_{+}\right), \mathrm{d} r ; \mathbb{C}^{M}\right)$, the function

$$
r \mapsto \int_{e_{-}}^{e_{+}} \frac{\bar{f}\left(r^{\prime}\right) \cdot g\left(r^{\prime}\right)}{r^{\prime}-r+\mathrm{io}} \mathrm{d} r^{\prime}
$$

is also in $\mathfrak{h}_{\mathcal{R}}$.

The main spectral and scattering theoretic results on $h_{\lambda}$ are given in the following Theorem which is an easy consequence of the techniques described in [Ja]. Its proof can be found in [JKP]. 
Theorem 6.2 Suppose that Assumptions (SEBB1), (SEBB2) and (SEBB4) hold. Then there exists a constant $\Lambda>0$ such that, for any $0<|\lambda|<\Lambda$ :

(i) The spectrum of $h_{\lambda}$ is purely absolutely continuous and equal to $\left[e_{-}, e_{+}\right]$.

(ii) The wave operators

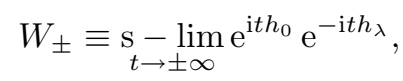

exist and are complete, i.e., $\operatorname{Ran} W_{ \pm}=\mathfrak{h}_{\mathcal{R}}$ and $W_{ \pm}: \mathfrak{h} \rightarrow \mathfrak{h}_{\mathcal{R}}$ are unitary. Moreover, if $\psi=\alpha \oplus g \in \mathfrak{h}$, then

$$
\left(W_{-} \psi\right)(r)=g(r)-\lambda F(r)^{-1}\left[\alpha-\lambda \int_{e_{-}}^{e_{+}} \frac{\bar{f}\left(r^{\prime}\right) \cdot g\left(r^{\prime}\right)}{r^{\prime}-r+\mathrm{i} o} \mathrm{~d} r^{\prime}\right] f(r) .
$$

Needless to say, the thermodynamics of the SEBB model can be studied under much more general assumptions than (SEBB1)-(SEBB4). However, these assumptions allow us to describe the results of [AJPP] with the least number of technicalities.

Parenthetically, we note that the SEBB model is obviously time-reversal invariant. Write $f_{j}(r)=\mathrm{e}^{\mathrm{i} \theta_{j}(r)}\left|f_{j}(r)\right|$, and let

$$
\mathfrak{j}\left(\alpha \oplus\left(g_{1}, \cdots, g_{M}\right)\right)=\bar{\alpha} \oplus\left(\mathrm{e}^{2 \mathrm{i} \theta_{1}} \bar{g}_{1}, \cdots, \mathrm{e}^{2 \mathrm{i} \theta_{M}} \bar{g}_{M}\right),
$$

where ${ }^{-}$denotes the usual complex conjugation. Then the map

$$
\mathfrak{r}(A)=\Gamma(\mathfrak{j}) A \Gamma\left(\mathfrak{j}^{-1}\right) .
$$

is a time reversal and $\omega$ is time reversal invariant.

Finally, as an example, consider a concrete SEBB model where each reservoir is a semi-infinite wire in the tight-binding approximation described in Example 5 of Subsection 5.2. Thus, for each $j, \mathfrak{h}_{\mathcal{R}_{j}}=\ell^{2}\left(\mathbb{Z}_{+}\right)$and $h_{\mathcal{R}_{j}}$ is the discrete Laplacian on $\mathbb{Z}_{+}$ with Dirichlet boundary condition at 0 . Choosing $f_{j}=\delta_{1}$ we obtain, in the spectral representation of $h_{\mathcal{R}_{j}}$,

$$
\begin{aligned}
\mathfrak{h}_{\mathcal{R}_{j}} & =L^{2}((-1,1), \mathrm{d} r), \\
h_{\mathcal{R}_{j}} & =r \\
f_{j}^{\#}(r) & =\sqrt{\frac{2}{\pi}}\left(1-r^{2}\right)^{1 / 4} .
\end{aligned}
$$

Thus, Assumptions (SEBB1) and (SEBB4) hold. Since, as $t \rightarrow \infty$, one has

$$
\int_{-1}^{1} \mathrm{e}^{\mathrm{i} t r}\left|f^{\#}(r)\right|^{2} \mathrm{~d} r=\frac{2 M}{t} J_{1}(t)=O\left(t^{-3 / 2}\right)
$$

where $J_{1}$ denotes a Bessel function of the first kind, Assumption (SEBB2) is also satisfied. Hence, if $\epsilon_{0} \in(-1,1)$, then the conclusions of Theorem 6.2 hold. In fact one can show that in this case

$$
\Lambda=\sqrt{\frac{1-\left|\varepsilon_{0}\right|}{2 M}} .
$$




\section{Thermodynamics of the SEBB model}

Throughout this and the next section we will assume that Assumptions (SEBB1)(SEBB4) hold.

\subsection{Non-equilibrium steady states}

In this subsection we show that the SEBB model has a unique NESS $\omega_{\lambda+}$ which does not depend on the choice of the initial state $\eta \in \mathcal{N}_{\omega}$. Recall that the reference state $\omega$ of the SEBB model is the quasi-free gauge-invariant state generated by $T=T_{\mathcal{S}} \oplus T_{\mathcal{R}}$, where $T_{\mathcal{S}}=\gamma \in(0,1)$ and $T_{\mathcal{R}}=\oplus_{j} \rho_{j}(r)$.

Theorem 7.1 Let $\Lambda>0$ be the constant introduced in Theorem 6.2. Then, for any real $\lambda$ such that $0<|\lambda|<\Lambda$ the following hold:

(i) The limit

$$
\alpha_{\lambda}^{+}(A) \equiv \lim _{t \rightarrow \infty} \tau_{0}^{-t} \circ \tau_{\lambda}^{t}(A)
$$

exists for all $A \in \mathcal{O}$. Moreover, $\operatorname{Ran} \alpha_{\lambda}^{+}=\mathcal{O}_{\mathcal{R}}$ and $\alpha_{\lambda}^{+}$is an isomorphism between the $C^{*}$-dynamical systems $\left(\mathcal{O}, \tau_{\lambda}\right)$ and $\left(\mathcal{O}_{\mathcal{R}}, \tau_{\mathcal{R}}\right)$.

(ii) Let $\omega_{\lambda+} \equiv \omega_{\mathcal{R}} \circ \alpha_{\lambda}^{+}$. Then

$$
\lim _{t \rightarrow \infty} \eta \circ \tau_{\lambda}^{t}=\omega_{\lambda+}
$$

for all $\eta \in \mathcal{N}_{\omega}$.

(iii) $\omega_{\lambda+}$ is the gauge-invariant quasi-free state on $\mathcal{O}$ generated by

$$
T_{+} \equiv W_{-}^{*} T_{\mathcal{R}} W_{-},
$$

where $W_{-}$is the wave operator of Theorem 6.2.

Proof. Recall that $\tau_{\lambda}^{t}$ is a group of Bogoliubov automorphisms, i.e., $\tau_{\lambda}^{t}\left(a^{\#}(f)\right)=$ $a^{\#}\left(\mathrm{e}^{\mathrm{i} t h_{\lambda}} f\right)$. Hence, for any observable of the form

$$
\begin{gathered}
A=a^{\#}\left(\psi_{1}\right) \cdots a^{\#}\left(\psi_{n}\right), \\
\tau_{0}^{-t} \circ \tau_{\lambda}^{t}(A)=a^{\#}\left(\mathrm{e}^{-\mathrm{i} t h_{0}} \mathrm{e}^{\mathrm{i} t h_{\lambda}} \psi_{1}\right) \cdots a^{\#}\left(\mathrm{e}^{-\mathrm{i} t h_{0}} \mathrm{e}^{\mathrm{i} t h_{\lambda}} \psi_{n}\right) .
\end{gathered}
$$

It follows from Theorem 6.2 that

$$
\lim _{t \rightarrow \infty} \tau_{0}^{-t} \circ \tau_{\lambda}^{t}(A)=a^{\#}\left(W_{-} \psi_{1}\right) \cdots a^{\#}\left(W_{-} \psi_{n}\right) .
$$

Since the linear span of set of elements of the form (7.52) is dense in $\mathcal{O}$, the limit (7.51) exists and is given by the Bogoliubov morphism $\alpha_{\lambda}^{+}\left(a^{\#}(f)\right)=a^{\#}\left(W_{-} f\right)$. Since $W_{-}$ is a unitary operator between $\mathfrak{h}$ and $\mathfrak{h}_{\mathcal{R}}$, $\operatorname{Ran} \alpha_{\lambda}^{+}=\operatorname{CAR}\left(\mathfrak{h}_{\mathcal{R}}\right)=\mathcal{O}_{\mathcal{R}}$, which proves Part (i). 
Since $h_{\mathcal{R}}$ has purely absolutely continuous spectrum, it follows from our discussion of quasi-free states in Subsection 5.1 that $\omega_{\mathcal{R}}$ is mixing for $\tau_{0}^{t}$. Part (ii) is thus a restatement of Proposition 3.9.

$$
\begin{aligned}
& \text { If } A=a^{*}\left(\psi_{n}\right) \cdots a^{*}\left(\psi_{1}\right) a\left(\phi_{1}\right) \cdots a\left(\phi_{m}\right) \text { is an element of } \mathcal{O}, \text { then } \\
& \qquad \begin{aligned}
\omega^{+}(A) & =\omega_{\mathcal{R}}\left(a^{*}\left(W_{-} \psi_{n}\right) \cdots a^{*}\left(W_{-} \psi_{1}\right) a\left(W_{-} \phi_{1}\right) \cdots a\left(W_{-} \phi_{m}\right)\right) \\
& =\delta_{n, m} \operatorname{det}\left\{\left(W_{-} \phi_{i}, T_{\mathcal{R}} W_{-} \psi_{j}\right)\right\} \\
& =\delta_{n, m} \operatorname{det}\left\{\left(\phi_{i}, T_{+} \psi_{j}\right)\right\} .
\end{aligned}
\end{aligned}
$$

and Part (iii) follows.

\subsection{The Hilbert-Schmidt condition}

Since $\omega$ and $\omega_{\lambda+}$ are factor states, they are either quasi-equivalent $\left(\mathcal{N}_{\omega}=\mathcal{N}_{\omega_{\lambda+}}\right)$ or disjoint $\left(\mathcal{N}_{\omega} \cap \mathcal{N}_{\omega_{\lambda+}}=\emptyset\right)$. Since $\operatorname{Ker} T=\operatorname{Ker}(I-T)=\{0\}$, we also have $\operatorname{Ker} T_{+}=\operatorname{Ker}\left(I-T_{+}\right)=\{0\}$, and so $\omega$ and $\omega_{\lambda_{+}}$are quasi-equivalent iff they are unitarily equivalent.

Let $\alpha>0$. A function $h:\left(e_{-}, e_{+}\right) \rightarrow \mathbb{C}$ is $\alpha$-Hölder continuous if there exists a constant $C$ such that for all $r, r^{\prime} \in\left(e_{-}, e_{+}\right),\left|h(r)-h\left(r^{\prime}\right)\right| \leq C\left|r-r^{\prime}\right|^{\alpha}$.

Theorem 7.2 Assume that all the densities $\rho_{j}(r)$ are the same and equal to $\rho(r)$. Assume further that the functions $\rho(r)^{1 / 2}$ and $(1-\rho(r))^{1 / 2}$ are $\alpha$-Hölder continuous for some $\alpha>1 / 2$. Then the operators

$$
\left(T_{+}\right)^{1 / 2}-T^{1 / 2} \quad \text { and } \quad\left(I-T_{+}\right)^{1 / 2}-(I-T)^{1 / 2}
$$

are Hilbert-Schmidt. In particular, the reference state $\omega$ and the NESS $\omega_{\lambda+}$ are unitarily equivalent and $\operatorname{Ep}\left(\omega_{\lambda+}\right)=0$.

Remark. We will prove this theorem in Appendix 9.2. Although the Hölder continuity assumption is certainly not optimal, it covers most cases of interest and allows for a technically simple proof.

Theorem 7.2 requires a comment. By the general principles of statistical mechanics, one expects that $\operatorname{Ep}\left(\omega_{\lambda_{+}}\right)=0$ if and only if all the reservoirs are in thermal equilibrium at the same inverse temperature $\beta$ and chemical potential $\mu$ (see Section 4.3 in [JP4]). This is not the case in the SEBB model because the perturbations $V_{j}$ are chosen in such a special way that the coupled dynamics is still given by a Bogoliubov automorphism. Following the strategy of [JP4], one can show that the Planck law $\rho(r)=\left(1+\mathrm{e}^{\beta(r-\mu)}\right)^{-1}$ can be deduced from the stability requirement $\operatorname{Ep}\left(\omega_{\lambda+}\right)=0$ for a more general class of interactions $V_{j}$. For reasons of space we will not discuss this subject in detail in these lecture notes (the interested reader may consult [AJPP]).

We will see below that the entropy production of the SEBB model is non-vanishing whenever the density operators of the reservoirs are not identical. 


\subsection{The heat and charge fluxes}

Recall that the observables describing heat and charge currents out of the $j$-th reservoir are

$$
\begin{aligned}
\Phi_{j} & =\lambda\left(a^{*}\left(\mathrm{i} r f_{j}\right) a(1)+a^{*}(1) a\left(\mathrm{i} r f_{j}\right)\right) \\
\mathcal{J}_{j} & =\lambda\left(a^{*}\left(\mathrm{i} f_{j}\right) a(1)+a^{*}(1) a\left(\mathrm{i} f_{j}\right)\right) .
\end{aligned}
$$

The expectation of the currents in the state $\omega_{\lambda+}$ are thus

$$
\begin{aligned}
\omega_{\lambda+}\left(\Phi_{j}\right) & =\mathrm{i} \lambda \omega_{\lambda+}\left(a^{*}\left(r f_{j}\right) a(1)-a^{*}(1) a\left(r f_{j}\right)\right) \\
& =2 \lambda \operatorname{Im}\left(r f_{j}, T_{+} 1\right) \\
& =2 \lambda \operatorname{Im}\left(W_{-} r f_{j}, T_{\mathcal{R}} W_{-} 1\right),
\end{aligned}
$$

and

$$
\begin{aligned}
\omega_{\lambda+}\left(\mathcal{J}_{j}\right) & =\mathrm{i} \lambda \omega_{\lambda+}\left(a^{*}\left(f_{j}\right) a(1)-a^{*}(1) a\left(f_{j}\right)\right) \\
& =2 \lambda \operatorname{Im}\left(f_{j}, T_{+} 1\right) \\
& =2 \lambda \operatorname{Im}\left(W_{-} f_{j}, T_{\mathcal{R}} W_{-} 1\right) .
\end{aligned}
$$

Setting

$$
G_{j}(r) \equiv \int_{e_{-}}^{e_{+}} \frac{r\left|f_{j}\left(r^{\prime}\right)\right|^{2}}{r^{\prime}-r+\mathrm{i} o} \mathrm{~d} r^{\prime}
$$

it easily follows from Formula (6.50) that for $k=1, \cdots, M$,

$$
\begin{aligned}
\left(T_{\mathcal{R}} W_{-} 1\right)_{k}(r) & =-\lambda \frac{\rho_{k}(r) f_{k}(r)}{F(r)} \\
\left(W_{-} r f_{j}\right)_{k}(r) & =\delta_{k j} r f_{j}(r)+\lambda^{2} \frac{G_{j}(r) f_{k}(r)}{F(r)},
\end{aligned}
$$

from which we obtain

$$
\left(W_{-} r f_{j}, T_{\mathcal{R}} W_{-} 1\right)=-\lambda \sum_{k=1}^{M} \int_{e_{-}}^{e_{+}} \frac{\left|f_{k}(r)\right|^{2} \rho_{k}(r)}{|F(r)|^{2}}\left[r \bar{F}(r) \delta_{k j}+\lambda^{2} \bar{G}_{j}(r)\right] \mathrm{d} r .
$$

From Equ. (6.49) we have $\operatorname{Im} \bar{F}(r)=-\lambda^{2} \pi|f(r)|^{2}$. Similarly $\operatorname{Im} \bar{G}_{j}(r)=\pi r\left|f_{j}(r)\right|^{2}$ and hence,

$$
\omega_{\lambda+}\left(\Phi_{j}\right)=2 \pi \lambda^{4} \sum_{k=1}^{M} \int_{e_{-}}^{e_{+}} \frac{r\left|f_{k}(r)\right|^{2} \rho_{k}(r)}{|F(r)|^{2}}\left[|f(r)|^{2} \delta_{k j}-\left|f_{j}(r)\right|^{2}\right] \mathrm{d} r .
$$

Since $|f|^{2}=\sum_{k}\left|f_{k}\right|^{2}$, the last formula can be rewritten as

$$
\omega_{\lambda+}\left(\Phi_{j}\right)=2 \pi \lambda^{4} \sum_{k=1}^{M} \int_{e_{-}}^{e_{+}}\left|f_{j}(r)\right|^{2}\left|f_{k}(r)\right|^{2}\left(\rho_{j}(r)-\rho_{k}(r)\right) \frac{r \mathrm{~d} r}{|F(r)|^{2}} .
$$


In a completely similar way one obtains

$$
\omega_{\lambda+}\left(\mathcal{J}_{j}\right)=2 \pi \lambda^{4} \sum_{k=1}^{M} \int_{e_{-}}^{e_{+}}\left|f_{j}(r)\right|^{2}\left|f_{k}(r)\right|^{2}\left(\rho_{j}(r)-\rho_{k}(r)\right) \frac{\mathrm{d} r}{|F(r)|^{2}} .
$$

An immediate consequence of Formulas (7.53) and (7.54) is that all the fluxes vanish if $\rho_{1}=\cdots=\rho_{M}$. Note also the antisymmetry in $k$ and $j$ of the integrands which ensures that the conservation laws

$$
\sum_{j=1}^{M} \omega_{\lambda+}\left(\Phi_{j}\right)=\sum_{j=1}^{M} \omega_{\lambda+}\left(\mathcal{J}_{j}\right)=0
$$

hold.

\subsection{Entropy production}

By the Assumption (SEBB3) the entropy production observable of the SEBB model is well defined and is given by Equ. (6.45) which we rewrite as

$$
\sigma=-\lambda \sum_{j=1}^{M}\left(a^{*}\left(\mathrm{i} s_{j} f_{j}\right) a(1)+a^{*}(1) a\left(\mathrm{i} s_{j} f_{j}\right)\right) .
$$

Proceeding as in the previous section we obtain

$$
\omega_{\lambda+}(\sigma)=-2 \lambda \sum_{j=1}^{M} \operatorname{Im}\left(W_{-} s_{j} f_{j}, T_{\mathcal{R}} W_{-} 1\right),
$$

which yields

$$
\omega_{\lambda+}(\sigma)=2 \pi \lambda^{4} \sum_{j, k=1}^{M} \int_{e_{-}}^{e_{+}} \frac{\left|f_{j}(r)\right|^{2}\left|f_{k}(r)\right|^{2}}{|F(r)|^{2}}\left(s_{j}(r)-s_{k}(r)\right) \rho_{k}(r) \mathrm{d} r .
$$

Finally, symmetrizing the sum over $j$ and $k$ we get

$$
\omega_{\lambda+}(\sigma)=\pi \lambda^{4} \sum_{j, k=1}^{M} \int_{e_{-}}^{e_{+}} \frac{\left|f_{j}(r)\right|^{2}\left|f_{k}(r)\right|^{2}}{|F(r)|^{2}}\left(s_{j}(r)-s_{k}(r)\right)\left(\rho_{k}(r)-\rho_{j}(r)\right) \mathrm{d} r .
$$

Since $\rho_{j}=\left(1+\mathrm{e}^{s_{j}}\right)^{-1}$ is a strictly decreasing function of $s_{j}$,

$$
\left(s_{j}(r)-s_{k}(r)\right)\left(\rho_{k}(r)-\rho_{j}(r)\right) \geq 0,
$$

with equality if and only if $\rho_{k}(r)=\rho_{j}(r)$. We summarize:

Theorem 7.3 The entropy production of $\omega_{\lambda+}$ is

$$
\omega_{\lambda+}(\sigma)=\pi \lambda^{4} \sum_{j, k=1}^{M} \int_{e_{-}}^{e_{+}} \frac{\left|f_{j}(r)\right|^{2}\left|f_{k}(r)\right|^{2}}{|F(r)|^{2}}\left(s_{j}(r)-s_{k}(r)\right)\left(\rho_{k}(r)-\rho_{j}(r)\right) \mathrm{d} r .
$$

In particular, $\operatorname{Ep}\left(\omega_{+}\right) \geq 0$ (something we already know from the general principles) and $\operatorname{Ep}\left(\omega_{+}\right)=0$ if and only if $\rho_{1}=\cdots=\rho_{M}$. 
Since $\omega$ and $\omega_{\lambda+}$ are factor states, they are either quasi-equivalent or disjoint. By Theorem 3.4, if $\operatorname{Ep}\left(\omega_{\lambda+}\right)>0$, then $\omega_{\lambda+}$ is not $\omega$-normal. Hence, Theorem 7.3 implies that if the densities $\rho_{j}$ are not all equal, then the reference state $\omega$ and the NESS $\omega_{\lambda+}$ are disjoint states.

Until the end of this section we will assume that the energy density of the $j$-th reservoir is

$$
\rho_{\beta_{j} \mu_{j}}(r) \equiv \frac{1}{1+\mathrm{e}^{\beta_{j}\left(r-\mu_{j}\right)}},
$$

where $\beta_{j}$ is the inverse temperature and $\mu_{j} \in \mathbb{R}$ is the chemical potential of the $j$-th reservoir. Then, by (6.46), $\operatorname{Ep}\left(\omega_{\lambda_{+}}\right)$can be written as

$$
\operatorname{Ep}\left(\omega_{\lambda+}\right)=\operatorname{Ep}_{\text {heat }}\left(\omega_{\lambda+}\right)+\operatorname{Ep}_{\text {charge }}\left(\omega_{\lambda+}\right)
$$

where

$$
\operatorname{Ep}_{\text {heat }}\left(\omega_{\lambda+}\right)=-\sum_{j=1}^{M} \beta_{j} \omega_{\lambda+}\left(\Phi_{j}\right),
$$

is interpreted as the entropy production due to the heat fluxes and

$$
\operatorname{Ep}_{\text {charge }}\left(\omega_{\lambda+}\right)=\sum_{j=1}^{M} \beta_{j} \mu_{j} \omega_{\lambda+}\left(\mathcal{J}_{j}\right) .
$$

as the entropy production due to the electric currents.

\subsection{Equilibrium correlation functions}

In this subsection we compute the integrated current-current correlation functions

$$
L_{\rho}(A, B) \equiv \lim _{T \rightarrow \infty} \frac{1}{2} \int_{-T}^{T} \omega_{\rho+}\left(\tau_{\lambda}^{t}(A) B\right) \mathrm{d} t,
$$

where $A$ and $B$ are heat or charge flux observables and $\omega_{\rho+}$ denotes the NESS $\omega_{\lambda+}$ in the equilibrium case $\rho_{1}=\cdots=\rho_{M}=\rho$. To do this, note that $\Phi_{l}=\mathrm{d} \Gamma\left(\varphi_{l}\right)$ and $\mathcal{J}_{l}=\mathrm{d} \Gamma\left(j_{l}\right)$ where

$$
\begin{aligned}
\varphi_{l} & =\mathrm{i}\left[h_{\mathcal{R}_{l}}, \lambda v\right]=-\mathrm{i}\left[h_{\lambda}, h_{\mathcal{R}_{j}}\right], \\
j_{l} & =\mathrm{i}\left[p_{j}, \lambda v\right]=-\mathrm{i}\left[h_{\lambda}, p_{j}\right],
\end{aligned}
$$

are finite rank operators. We will only consider $L_{\rho}\left(\Phi_{j}, \Phi_{k}\right)$, the other cases are completely similar.

Using the CAR, Formula (5.29) and the fact that $\omega_{\rho+}\left(\Phi_{l}\right)=0$, one easily shows that

$$
\omega_{\rho+}\left(\tau_{\lambda}^{t}\left(\Phi_{j}\right) \Phi_{k}\right)=\operatorname{Tr}\left(T_{+} \mathrm{e}^{\mathrm{i} t h_{\lambda}} \varphi_{j} \mathrm{e}^{-\mathrm{i} t h_{\lambda}}\left(I-T_{+}\right) \varphi_{k}\right) .
$$

Since

$$
\mathrm{e}^{\mathrm{i} t h_{\lambda}} \varphi_{j} \mathrm{e}^{-\mathrm{i} t h_{\lambda}}=-\frac{\mathrm{d}}{\mathrm{d} t} \mathrm{e}^{\mathrm{i} t h_{\lambda}} h_{\mathcal{R}_{j}} \mathrm{e}^{-\mathrm{i} t h_{\lambda}}
$$


the integration can be explicitly performed and we have

$$
L_{\rho}\left(\Phi_{j}, \Phi_{k}\right)=-\left.\lim _{T \rightarrow \infty} \frac{1}{2} \operatorname{Tr}\left(T_{+} \mathrm{e}^{\mathrm{i} t h_{\lambda}} h_{\mathcal{R}_{j}} \mathrm{e}^{-\mathrm{i} t h_{\lambda}}\left(I-T_{+}\right) \varphi_{k}\right)\right|_{-T} ^{T} .
$$

Writing $\mathrm{e}^{\mathrm{i} t h_{\lambda}} h_{\mathcal{R}_{j}} \mathrm{e}^{-\mathrm{i} t h_{\lambda}}=\mathrm{e}^{\mathrm{i} t h_{\lambda}} \mathrm{e}^{-\mathrm{i} t h_{0}} h_{\mathcal{R}_{j}} \mathrm{e}^{\mathrm{i} t h_{0}} \mathrm{e}^{-\mathrm{i} t h_{\lambda}}$ and using the fact that $\varphi_{k}$ is finite rank, we see that the limit exists and can be expressed in terms of the wave operators $W_{ \pm}$as

$$
\begin{aligned}
L_{\rho}\left(\Phi_{j}, \Phi_{k}\right)= & \frac{1}{2}\left\{\operatorname{Tr}\left(T_{+} W_{-}^{*} h_{\mathcal{R}_{j}} W_{-}\left(I-T_{+}\right) \varphi_{k}\right)\right. \\
& \left.-\operatorname{Tr}\left(T_{+} W_{+}^{*} h_{\mathcal{R}_{j}} W_{+}\left(I-T_{+}\right) \varphi_{k}\right)\right\} .
\end{aligned}
$$

The intertwining property of the wave operators gives

$$
T_{+}=W_{-}^{*} \rho\left(h_{\mathcal{R}}\right) W_{-}=\rho\left(h_{\lambda}\right)=W_{+}^{*} \rho\left(h_{\mathcal{R}}\right) W_{+},
$$

from which we obtain

$$
L_{\rho}\left(\Phi_{j}, \Phi_{k}\right)=\frac{1}{2} \operatorname{Tr}\left(T_{\mathcal{R}}\left(I-T_{\mathcal{R}}\right) h_{\mathcal{R}_{j}}\left(W_{-} \varphi_{k} W_{-}^{*}-W_{+} \varphi_{k} W_{+}^{*}\right)\right),
$$

with $T_{\mathcal{R}}=\rho\left(h_{\mathcal{R}}\right)$. Time reversal invariance further gives

$$
W_{+}=\mathfrak{j} W_{-} \mathfrak{j}, \quad \mathfrak{j} \varphi_{k} \mathfrak{j}=-\varphi_{k},
$$

and so

$$
\begin{aligned}
L_{\rho}\left(\Phi_{j}, \Phi_{k}\right) & =\frac{1}{2} \operatorname{Tr}\left(T_{\mathcal{R}}\left(I-T_{\mathcal{R}}\right) h_{\mathcal{R}_{j}}\left(W_{-} \varphi_{k} W_{-}^{*}+\mathfrak{j} W_{-} \varphi_{k} W_{-}^{*} \mathfrak{j}\right)\right) \\
& =\operatorname{Tr}\left(T_{\mathcal{R}}\left(I-T_{\mathcal{R}}\right) h_{\mathcal{R}_{j}} W_{-} \varphi_{k} W_{-}^{*}\right) .
\end{aligned}
$$

The last trace is easily evaluated (use the formula $\varphi_{k}=\lambda \mathrm{i}\left[h_{\mathcal{R}_{k}}, v\right]$ and follow the steps of the computation in Subsection 7.3). The result is

$$
\begin{aligned}
& L_{\rho}\left(\Phi_{j}, \Phi_{k}\right)=-2 \pi \lambda^{4} \int_{e_{-}}^{e_{+}}\left|f_{j}(r)\right|^{2}\left[\left|f_{k}(r)\right|^{2}-\delta_{j k}|f(r)|^{2}\right] \rho(r)(1-\rho(r)) \frac{r^{2} \mathrm{~d} r}{|F(r)|^{2}} \\
& L_{\rho}\left(\mathcal{J}_{j}, \Phi_{k}\right)=-2 \pi \lambda^{4} \int_{e_{-}}^{e_{+}}\left|f_{j}(r)\right|^{2}\left[\left|f_{k}(r)\right|^{2}-\delta_{j k}|f(r)|^{2}\right] \rho(r)(1-\rho(r)) \frac{r \mathrm{~d} r}{|F(r)|^{2}} \\
& L_{\rho}\left(\Phi_{j}, \mathcal{J}_{k}\right)=-2 \pi \lambda^{4} \int_{e_{-}}^{e_{+}}\left|f_{j}(r)\right|^{2}\left[\left|f_{k}(r)\right|^{2}-\delta_{j k}|f(r)|^{2}\right] \rho(r)(1-\rho(r)) \frac{r \mathrm{~d} r}{|F(r)|^{2}} \\
& L_{\rho}\left(\mathcal{J}_{j}, \mathcal{J}_{k}\right)=-2 \pi \lambda^{4} \int_{e_{-}}^{e_{+}}\left|f_{j}(r)\right|^{2}\left[\left|f_{k}(r)\right|^{2}-\delta_{j k}|f(r)|^{2}\right] \rho(r)(1-\rho(r)) \frac{\mathrm{d} r}{|F(r)|^{2}}
\end{aligned}
$$

Note the following symmetries:

$$
\begin{aligned}
& L_{\rho}\left(\Phi_{j}, \Phi_{k}\right)=L_{\rho}\left(\Phi_{k}, \Phi_{j}\right), \\
& L_{\rho}\left(\mathcal{J}_{j}, \mathcal{J}_{k}\right)=L_{\rho}\left(\mathcal{J}_{k}, \mathcal{J}_{j}\right), \\
& L_{\rho}\left(\Phi_{j}, \mathcal{J}_{k}\right)=L_{\rho}\left(\mathcal{J}_{k}, \Phi_{j}\right) .
\end{aligned}
$$

Note also that $L_{\rho}\left(\Phi_{j}, \Phi_{k}\right) \leq 0$ and $L_{\rho}\left(\mathcal{J}_{j}, \mathcal{J}_{k}\right) \leq 0$ for $j \neq k$ while $L_{\rho}\left(\Phi_{j}, \Phi_{j}\right) \geq 0$ and $L_{\rho}\left(\mathcal{J}_{j}, \mathcal{J}_{j}\right) \geq 0$. 


\subsection{Onsager relations. Kubo formulas.}

Let $\beta_{\text {eq }}$ and $\mu_{\text {eq }}$ be given equilibrium values of the inverse temperature and the chemical potential. The affinities (thermodynamic forces) conjugated to the currents $\Phi_{j}$ and $\mathcal{J}_{j}$ are

$$
X_{j}=\beta_{\text {eq }}-\beta_{j}, \quad Y_{j}=\beta_{j} \mu_{j}-\beta_{\text {eq }} \mu_{\text {eq }} .
$$

Indeed, it follows from the conservations laws (4.12) and (6.39) that

$$
\operatorname{Ep}\left(\omega_{\lambda+}\right)=\sum_{j=1}^{M}\left(X_{j} \omega_{\lambda+}\left(\Phi_{j}\right)+Y_{j} \omega_{\lambda+}\left(\mathcal{J}_{j}\right)\right) \text {. }
$$

Since

we have

$$
\rho_{\beta_{j} \mu_{j}}(r)=\frac{1}{1+\mathrm{e}^{\beta_{\mathrm{eq}}\left(r-\mu_{\mathrm{eq}}\right)-\left(X_{j} r+Y_{j}\right)}},
$$

$$
\begin{aligned}
&\left.\partial_{X_{k}} \rho_{\beta_{j} \mu_{j}}(r)\right|_{X=Y=0}=\delta_{k j} \rho(r)(1-\rho(r)) r, \\
&\left.\partial_{Y_{k}} \rho_{\beta_{j} \mu_{j}}(r)\right|_{X=Y=0}=\delta_{k j} \rho(r)(1-\rho(r)),
\end{aligned}
$$

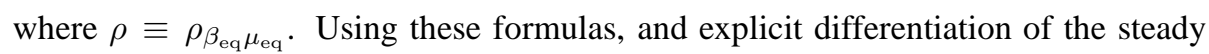
currents (7.53) and (7.54) and comparison with (7.56) lead to

$$
\begin{aligned}
\left.\partial_{X_{k}} \omega_{\lambda+}\left(\Phi_{j}\right)\right|_{X=Y=0} & =L_{\rho}\left(\Phi_{j}, \Phi_{k}\right), \\
\left.\partial_{Y_{k}} \omega_{\lambda+}\left(\Phi_{j}\right)\right|_{X=Y=0} & =L_{\rho}\left(\Phi_{j}, \mathcal{J}_{k}\right), \\
\left.\partial_{X_{k}} \omega_{\lambda+}\left(\mathcal{J}_{j}\right)\right|_{X=Y=0} & =L_{\rho}\left(\mathcal{J}_{j}, \Phi_{k}\right), \\
\left.\partial_{Y_{k}} \omega_{\lambda+}\left(\mathcal{J}_{j}\right)\right|_{X=Y=0} & =L_{\rho}\left(\mathcal{J}_{j}, \mathcal{J}_{k}\right),
\end{aligned}
$$

which are the Kubo Fluctuation-Dissipation Formulas. The symmetry (7.57) gives the Onsager reciprocity relations

$$
\begin{aligned}
\left.\partial_{X_{j}} \omega_{\lambda+}\left(\Phi_{k}\right)\right|_{X=Y=0} & =\left.\partial_{X_{k}} \omega_{\lambda+}\left(\Phi_{j}\right)\right|_{X=Y=0} \\
\left.\partial_{Y_{j}} \omega_{\lambda+}\left(\mathcal{J}_{k}\right)\right|_{X=Y=0} & =\left.\partial_{Y_{k}} \omega_{\lambda+}\left(\mathcal{J}_{j}\right)\right|_{X=Y=0} \\
\left.\partial_{Y_{j}} \omega_{\lambda+}\left(\Phi_{k}\right)\right|_{X=Y=0} & =\left.\partial_{X_{k}} \omega_{\lambda+}\left(\mathcal{J}_{j}\right)\right|_{X=Y=0} .
\end{aligned}
$$

The fact that $L_{\rho}\left(\Phi_{j}, \Phi_{j}\right) \geq 0$ and $L_{\rho}\left(\mathcal{J}_{j}, \mathcal{J}_{j}\right) \geq 0$ while $L_{\rho}\left(\Phi_{j}, \Phi_{k}\right) \leq 0$ and $L_{\rho}\left(\mathcal{J}_{j}, \mathcal{J}_{k}\right) \leq 0$ for $j \neq k$ means that increasing a force results in an increase of the conjugated current and a decrease of the other currents. This is not only true in the linear regime. Direct differentiation of (7.53) and (7.54) yields

$$
\begin{aligned}
\partial_{X_{k}} \omega_{\lambda+}\left(\Phi_{k}\right) & =2 \pi \lambda^{4} \sum_{j \neq k} \int_{e_{-}}^{e_{+}}\left|f_{j}(r)\right|^{2}\left|f_{k}(r)\right|^{2} \rho_{\beta_{k} \mu_{k}}(r)\left(1-\rho_{\beta_{k} \mu_{k}}(r)\right) \frac{r^{2} \mathrm{~d} r}{|F(r)|^{2}} \geq 0 \\
\partial_{Y_{k}} \omega_{\lambda+}\left(\mathcal{J}_{k}\right) & =2 \pi \lambda^{4} \sum_{j \neq k} \int_{e_{-}}^{e_{+}}\left|f_{j}(r)\right|^{2}\left|f_{k}(r)\right|^{2} \rho_{\beta_{k} \mu_{k}}(r)\left(1-\rho_{\beta_{k} \mu_{k}}(r)\right) \frac{\mathrm{d} r}{|F(r)|^{2}} \geq 0 \\
\partial_{X_{k}} \omega_{\lambda+}\left(\Phi_{j}\right) & =-2 \pi \lambda^{4} \int_{e_{-}}^{e_{+}}\left|f_{j}(r)\right|^{2}\left|f_{k}(r)\right|^{2} \rho_{\beta_{k} \mu_{k}}(r)\left(1-\rho_{\beta_{k} \mu_{k}}(r)\right) \frac{r^{2} \mathrm{~d} r}{|F(r)|^{2}} \leq 0 \\
\partial_{Y_{k}} \omega_{\lambda+}\left(\mathcal{J}_{j}\right) & =-2 \pi \lambda^{4} \int_{e_{-}}^{e_{+}}\left|f_{j}(r)\right|^{2}\left|f_{k}(r)\right|^{2} \rho_{\beta_{k} \mu_{k}}(r)\left(1-\rho_{\beta_{k} \mu_{k}}(r)\right) \frac{\mathrm{d} r}{|F(r)|^{2}} \leq 0 .
\end{aligned}
$$


Note that these derivatives do not depend on the reference states of the reservoirs $\mathcal{R}_{j}$ for $j \neq k$.

\section{FGR thermodynamics of the SEBB model}

For $j=1, \cdots, M$, we set

$$
\tilde{g}_{j}(t) \equiv \int_{e_{-}}^{e_{+}} \mathrm{e}^{\mathrm{i} t r} \rho_{j}(r)\left|f_{j}(r)\right|^{2} \mathrm{~d} r .
$$

In addition to (SEBB1)-(SEBB4) in this section we will assume

Assumption (SEBB5) $\tilde{g}_{j}(t) \in L^{1}(\mathbb{R}, \mathrm{d} t)$ for $j=1, \cdots, M$.

\subsection{The weak coupling limit}

In this subsection we study the dynamics restricted to the small system on the van Hove time scale $t / \lambda^{2}$.

Recall that by Theorem 6.1 the algebra of observables $\mathcal{O}_{\mathcal{S}}$ of the small system is the 4-dimensional subalgebra of $\mathcal{O}=\operatorname{CAR}\left(\mathbb{C} \oplus \mathfrak{h}_{\mathcal{R}}\right)$ generated by $a(1)$. It is the full matrix algebra of the subspace $\mathfrak{h}_{\mathcal{S}} \subset \Gamma_{-}\left(\mathbb{C} \oplus \mathfrak{h}_{\mathcal{R}}\right)$ generated by the vectors $\{\Omega, a(1) \Omega\}$. In this basis, the Hamiltonian and the reference state of the small system are

$$
H_{\mathcal{S}}=\left[\begin{array}{cc}
0 & 0 \\
0 & \varepsilon_{0}
\end{array}\right], \quad \omega_{\mathcal{S}}=\left[\begin{array}{cc}
1-\gamma & 0 \\
0 & \gamma
\end{array}\right]
$$

Let $A \in \mathcal{O}_{\mathcal{S}}$ be an observable of the small system. We will study the expectation values

$$
\omega\left(\tau_{\lambda}^{t / \lambda^{2}}(A)\right)
$$

as $\lambda \rightarrow 0$. If $A=a^{\#}(1)$, then (8.58) vanishes, so we need only to consider the Abelian 2-dimensional even subalgebra $\mathcal{O}_{\mathcal{S}}^{+} \subset \mathcal{O}_{\mathcal{S}}$. Since $a^{*}(1) a(1)=N_{\mathcal{S}}$ and $a(1) a^{*}(1)=$ $I-N_{\mathcal{S}}$, it suffices to consider $A=N_{\mathcal{S}}$. In this case we have

$$
\begin{aligned}
\omega \circ \tau_{\lambda}^{t / \lambda^{2}}\left(N_{\mathcal{S}}\right) & =\omega\left(a^{*}\left(\mathrm{e}^{\mathrm{i} t h_{\lambda} / \lambda^{2}} 1\right) a\left(\mathrm{e}^{\mathrm{i} t h_{\lambda} / \lambda^{2}} 1\right)\right) \\
& =\left(\mathrm{e}^{\mathrm{i} t h_{\lambda} / \lambda^{2}} 1,\left(\gamma \oplus T_{\mathcal{R}}\right) \mathrm{e}^{\mathrm{i} t h_{\lambda} / \lambda^{2}} 1\right) .
\end{aligned}
$$

Using the projection $p_{j}$ on the Hilbert space $\mathfrak{h}_{\mathcal{R}_{j}}$ of the $j$-th reservoir we can rewrite this expression as

$$
\omega \circ \tau_{\lambda}^{t / \lambda^{2}}\left(N_{\mathcal{S}}\right)=\gamma\left|\left(1, \mathrm{e}^{\mathrm{i} t h_{\lambda} / \lambda^{2}} 1\right)\right|^{2}+\sum_{j=1}^{M}\left(p_{j} \mathrm{e}^{\mathrm{i} t h_{\lambda} / \lambda^{2}} 1, T_{\mathcal{R}_{j}} p_{j} \mathrm{e}^{\mathrm{i} t h_{\lambda} / \lambda^{2}} 1\right) .
$$

Theorem 8.1 Assume that Assumptions (SEBB1)-(SEBB5) hold. 
(i) For any $t \geq 0$,

$$
\lim _{\lambda \rightarrow 0}\left|\left(1, \mathrm{e}^{\mathrm{i} t h_{\lambda} / \lambda^{2}} 1\right)\right|^{2}=\mathrm{e}^{-2 \pi t\left|f\left(\varepsilon_{0}\right)\right|^{2}} .
$$

(ii) For any $t \geq 0$ and $j=1, \cdots, M$,

$$
\lim _{\lambda \rightarrow 0}\left(p_{j} \mathrm{e}^{\mathrm{i} t h_{\lambda} / \lambda^{2}} 1, T_{\mathcal{R}_{j}} p_{j} \mathrm{e}^{\mathrm{i} t h_{\lambda} / \lambda^{2}} 1\right)=\frac{\left|f_{j}\left(\varepsilon_{0}\right)\right|^{2}}{\left|f\left(\varepsilon_{0}\right)\right|^{2}} \rho_{j}\left(\varepsilon_{0}\right)\left(1-\mathrm{e}^{-2 \pi t\left|f\left(\varepsilon_{0}\right)\right|^{2}}\right) .
$$

The proof of Theorem 8.1 is not difficult—for Part (i) see [Da1, D1], and for Part (ii) [Da2]. These proofs use the regularity Assumption (SEBB5). An alternative proof of Theorem 8.1, based on the explicit form of the wave operator $W_{-}$, can be found in [JKP].

Theorem 8.1 implies that

$$
\begin{aligned}
\gamma(t) & \equiv \lim _{\lambda \rightarrow 0} \omega \circ \tau_{\lambda}^{t / \lambda^{2}}\left(N_{\mathcal{S}}\right) \\
& =\gamma \mathrm{e}^{-2 \pi t\left|f\left(\varepsilon_{0}\right)\right|^{2}}+\left(1-\mathrm{e}^{-2 \pi t\left|f\left(\varepsilon_{0}\right)\right|^{2}}\right) \sum_{j=1}^{M} \frac{\left|f_{j}\left(\varepsilon_{0}\right)\right|^{2}}{\left|f\left(\varepsilon_{0}\right)\right|^{2}} \rho_{j}\left(\varepsilon_{0}\right),
\end{aligned}
$$

from which we easily conclude that for all $A \in \mathcal{O}_{\mathcal{S}}$ one has

$$
\lim _{\lambda \rightarrow 0} \omega \circ \tau_{\lambda}^{t / \lambda^{2}}(A)=\operatorname{Tr}\left(\omega_{\mathcal{S}}(t) A\right),
$$

where

$$
\omega_{\mathcal{S}}(t)=\left[\begin{array}{cc}
1-\gamma(t) & 0 \\
0 & \gamma(t)
\end{array}\right]
$$

According to the general theory described in Section 4.5 we also have

$$
\omega_{\mathcal{S}}(t)=\mathrm{e}^{t K_{\mathrm{S}}} \omega_{\mathcal{S}}
$$

where $K_{\mathrm{S}}$ is the QMS generator in the Schrödinger picture. We shall now discuss its restriction to the algebra of diagonal $2 \times 2$-matrices. In the basis

$$
\left[\begin{array}{ll}
1 & 0 \\
0 & 0
\end{array}\right],\left[\begin{array}{ll}
0 & 0 \\
0 & 1
\end{array}\right]
$$

of this subalgebra we obtain the matrix representation

$$
K_{\mathrm{S}}=2 \pi \sum_{j=1}^{M}\left|f_{j}\left(\varepsilon_{0}\right)\right|^{2}\left[\begin{array}{cc}
-\rho_{j}\left(\varepsilon_{0}\right) & 1-\rho_{j}\left(\varepsilon_{0}\right) \\
\rho_{j}\left(\varepsilon_{0}\right) & -\left(1-\rho_{j}\left(\varepsilon_{0}\right)\right)
\end{array}\right] .
$$

In the Heisenberg picture we have

$$
\lim _{\lambda \rightarrow 0} \omega_{\mathcal{S}} \circ \tau_{\lambda}^{t / \lambda^{2}}(A)=\operatorname{Tr}\left(\omega_{\mathcal{S}} \mathrm{e}^{t K_{\mathrm{H}}} A\right)
$$


where $K_{\mathrm{H}}$ is related to $K_{\mathrm{S}}$ by the duality

$$
\operatorname{Tr}\left(K_{\mathrm{S}}\left(\omega_{\mathcal{S}}\right) A\right)=\operatorname{Tr}\left(\omega_{\mathcal{S}} K_{\mathrm{H}}(A)\right) .
$$

The restriction of $K_{\mathrm{H}}$ to the subalgebra of diagonal $2 \times 2$-matrices has the following matrix representation relative to the basis (8.62),

$$
K_{\mathrm{H}}=2 \pi \sum_{j=1}^{M}\left|f_{j}\left(\varepsilon_{0}\right)\right|^{2}\left[\begin{array}{cc}
-\rho_{j}\left(\varepsilon_{0}\right) & \rho_{j}\left(\varepsilon_{0}\right) \\
1-\rho_{j}\left(\varepsilon_{0}\right) & -\left(1-\rho_{j}\left(\varepsilon_{0}\right)\right)
\end{array}\right] .
$$

We stress that $K_{\mathrm{S}}$ and $K_{\mathrm{H}}$ are the diagonal parts of the full Davies generators in the Schrödinger and Heisenberg pictures discussed in the lecture notes [D1].

As we have discussed in Section 4.5, an important property of the generators $K_{\mathrm{S}}$ and $K_{\mathrm{H}}$ is the decomposition

$$
K_{\mathrm{S}}=\sum_{j=1}^{M} K_{\mathrm{S}, j}, \quad K_{\mathrm{H}}=\sum_{j=1}^{M} K_{\mathrm{H}, j},
$$

where $K_{\mathrm{S}, j}$ and $K_{\mathrm{H}, j}$ are the generators describing interaction of $\mathcal{S}$ with the $j$-th reservoir only. Explicitly,

$$
\begin{aligned}
K_{\mathrm{S}, j} & =2 \pi\left|f_{j}\left(\varepsilon_{0}\right)\right|^{2}\left[\begin{array}{cc}
-\rho_{j}\left(\varepsilon_{0}\right) & 1-\rho_{j}\left(\varepsilon_{0}\right) \\
\rho_{j}\left(\varepsilon_{0}\right) & -\left(1-\rho_{j}\left(\varepsilon_{0}\right)\right)
\end{array}\right], \\
K_{\mathrm{H}, j} & =2 \pi\left|f_{j}\left(\varepsilon_{0}\right)\right|^{2}\left[\begin{array}{cc}
-\rho_{j}\left(\varepsilon_{0}\right) & \rho_{j}\left(\varepsilon_{0}\right) \\
1-\rho_{j}\left(\varepsilon_{0}\right) & -\left(1-\rho_{j}\left(\varepsilon_{0}\right)\right)
\end{array}\right] .
\end{aligned}
$$

Finally, we note that

$$
\omega_{\mathcal{S}+} \equiv \lim _{t \rightarrow \infty} \omega_{\mathcal{S}}(t)=\sum_{j=1}^{M} \frac{\left|f_{j}\left(\varepsilon_{0}\right)\right|^{2}}{\left|f\left(\varepsilon_{0}\right)\right|^{2}}\left[\begin{array}{cc}
1-\rho_{j}\left(\varepsilon_{0}\right) & 0 \\
0 & \rho_{j}\left(\varepsilon_{0}\right)
\end{array}\right] .
$$

$\omega_{\mathcal{S}+}$ is the NESS on the Fermi Golden Rule time scale: for any observable $A$ of the small system,

$$
\lim _{t \rightarrow \infty} \lim _{\lambda \rightarrow 0} \omega \circ \tau_{\lambda}^{t / \lambda^{2}}(A)=\operatorname{Tr}\left(\omega_{\mathcal{S}+} A\right)=\omega_{\mathcal{S}+}(A) .
$$

In the sequel we will refer to $\omega_{\mathcal{S}+}$ as the FGR NESS.

\subsection{Historical digression-Einstein's derivation of the Planck law}

Einstein's paper [Ei], published in 1917, has played an important role in the historical development of quantum mechanics and quantum field theory. In this paper Einstein made some deep insights into the nature of interaction between radiation and matter which have led him to a new derivation of the Planck law. For the history of these early developments the interested reader may consult [Pa]. 
The original Einstein argument can be paraphrased as follows. Consider a twolevel quantum system $\mathcal{S}$ with energy levels 0 and $\varepsilon_{0}$, which is in equilibrium with a radiation field reservoir with energy density $\rho(r)$. Due to the interaction with the reservoir, the system $\mathcal{S}$ will make constant transitions between the energy levels 0 and $\varepsilon_{0}$. Einstein conjectured that the corresponding transition rates (transition probabilities per unit time) have the form

$$
k\left(\varepsilon_{0}, 0\right)=A_{\varepsilon_{0}}\left(1-\rho\left(\varepsilon_{0}\right)\right), \quad k\left(0, \varepsilon_{0}\right)=B_{\varepsilon_{0}} \rho\left(\varepsilon_{0}\right),
$$

where $A_{\varepsilon_{0}}$ and $B_{\varepsilon_{0}}$ are the coefficients which depend on the mechanics of the interaction. (Of course, in 1917 Einstein considered the bosonic reservoir (the light)—in this case in the first formula one has $1+\rho\left(\varepsilon_{0}\right)$ instead of $\left.1-\rho\left(\varepsilon_{0}\right)\right)$. These formulas are the celebrated Einstein's $A$ and $B$ laws. Let $\bar{p}_{0}$ and $\bar{p}_{\varepsilon_{0}}$ be probabilities that in equilibrium the small system has energies 0 and $\varepsilon_{0}$ respectively. If $\mathcal{S}$ is in thermal equilibrium at inverse temperature $\beta$, then by the Gibbs postulate,

$$
\bar{p}_{0}=\left(1+\mathrm{e}^{-\beta \varepsilon_{0}}\right)^{-1}, \quad \bar{p}_{\varepsilon_{0}}=\mathrm{e}^{-\beta \varepsilon_{0}}\left(1+\mathrm{e}^{-\beta \varepsilon_{0}}\right)^{-1} .
$$

The equilibrium condition

$$
k\left(0, \varepsilon_{0}\right) \bar{p}_{0}=k\left(\varepsilon_{0}, 0\right) \bar{p}_{\varepsilon_{0}}
$$

yields

$$
\rho\left(\varepsilon_{0}\right)=\frac{A_{\varepsilon_{0}}}{B_{\varepsilon_{0}}}\left(1-\rho\left(\varepsilon_{0}\right)\right) \mathrm{e}^{-\beta \varepsilon_{0}} .
$$

In 1917 Einstein naturally could not compute the coefficients $A_{\varepsilon_{0}}$ and $B_{\varepsilon_{0}}$. However, if $A_{\varepsilon_{0}} / B_{\varepsilon_{0}}=1$ for all $\varepsilon_{0}$, then the above relation yields the Planck law for energy density of the free fermionic reservoir in thermal equilibrium,

$$
\rho\left(\varepsilon_{0}\right)=\frac{1}{1+\mathrm{e}^{\beta \varepsilon_{0}}} .
$$

In his paper Einstein points out that to compute the numerical value of $A_{\varepsilon_{0}}$ and $B_{\varepsilon_{0}}$ one would need an exact [quantum] theory of electro-dynamical and mechanical processes.

The quantum theory of mechanical processes was developed in the 1920's by Heisenberg, Schrödinger, Jordan, Dirac and others. In 1928, Dirac extended quantum theory to electrodynamical processes and computed the coefficients $A_{\varepsilon_{0}}$ and $B_{\varepsilon_{0}}$ from the first principles of quantum theory. Dirac's seminal paper [Di] marked the birth of quantum field theory. To compute $A_{\varepsilon_{0}}$ and $B_{\varepsilon_{0}}$ Dirac developed the so-called timedependent perturbation theory, which has been discussed in lecture notes [D1, JKP] (see also Chapter XXI in [Mes], or any book on quantum mechanics). In his 1949 Chicago lecture notes [Fer] Fermi called the basic formulas of Dirac's theory the Golden Rule, and since then they have been called the Fermi Golden Rule.

In this section we have described the mathematically rigorous Fermi Golden Rule theory of the SEBB model. In this context Dirac's theory reduces to the computation of $K_{\mathrm{S}}$ and $K_{\mathrm{H}}$ since the matrix elements of these operators give the transition probabilities $k\left(\varepsilon_{0}, 0\right)$ and $k\left(0, \varepsilon_{0}\right)$. In particular, in the case of a single reservoir with energy density $\rho(r)$,

$$
A_{\varepsilon_{0}}=B_{\varepsilon_{0}}=2 \pi\left|f\left(\varepsilon_{0}\right)\right|^{2} .
$$


Einstein's argument can be rephrased as follows: if the energy density $\rho$ is such that

$$
\omega_{\mathcal{S}+}=\mathrm{e}^{-\beta H_{\mathcal{S}}} / \operatorname{Tr}\left(\mathrm{e}^{-\beta H_{\mathcal{S}}}\right)=\left(1+\mathrm{e}^{-\beta \varepsilon_{0}}\right)^{-1}\left[\begin{array}{cc}
1 & 0 \\
0 & \mathrm{e}^{-\beta \varepsilon_{0}}
\end{array}\right],
$$

for all $\varepsilon_{0}\left(\right.$ namely $\left.H_{\mathcal{S}}\right)$, then

$$
\rho\left(\varepsilon_{0}\right)=\frac{1}{1+\mathrm{e}^{\beta \varepsilon_{0}}} .
$$

\subsection{FGR fluxes, entropy production and Kubo formulas}

Any diagonal observable $A \in \mathcal{O}_{\mathcal{S}}^{+}$of the small system is a function of the Hamiltonian $H_{\mathcal{S}}$. We identify such an observable with a function $g:\left\{0, \varepsilon_{0}\right\} \rightarrow \mathbb{R}$. Occasionally, we will write $g$ as a column vector with components $g(0)$ and $g\left(\varepsilon_{0}\right)$. In the sequel we will use such identifications without further comment. A vector $\nu$ is called a probability vector if $\nu(0) \geq 0, \nu\left(\varepsilon_{0}\right) \geq 0$ and $\nu(0)+\nu\left(\varepsilon_{0}\right)=1$. The diagonal part of any density matrix defines a probability vector. We denote the probability vector associated to FGR NESS $\omega_{\mathcal{S}}+$ by the same letter. Similarly, to a probability vector one uniquely associates a diagonal density matrix. With these conventions, the Hamiltonian and the number operator of the small system are

$$
H_{\mathcal{S}}=\varepsilon_{0} a^{*}(1) a(1)=\left[\begin{array}{c}
0 \\
\varepsilon_{0}
\end{array}\right], \quad N_{\mathcal{S}}=a^{*}(1) a(1)=\left[\begin{array}{l}
0 \\
1
\end{array}\right]
$$

The Fermi Golden Rule (FGR) heat and charge flux observables are

$$
\begin{aligned}
\Phi_{\mathrm{fgr}, j} & =K_{\mathrm{H}, j}\left(H_{\mathcal{S}}\right)=2 \pi \varepsilon_{0}\left|f_{j}\left(\varepsilon_{0}\right)\right|^{2}\left[\begin{array}{c}
\rho_{j}\left(\varepsilon_{0}\right) \\
-\left(1-\rho_{j}\left(\varepsilon_{0}\right)\right)
\end{array}\right], \\
\mathcal{J}_{\mathrm{fgr}, j} & =K_{\mathrm{H}, j}\left(N_{\mathcal{S}}\right)=2 \pi\left|f_{j}\left(\varepsilon_{0}\right)\right|^{2}\left[\begin{array}{c}
\rho_{j}\left(\varepsilon_{0}\right) \\
-\left(1-\rho_{j}\left(\varepsilon_{0}\right)\right)
\end{array}\right] .
\end{aligned}
$$

The steady heat and the charge currents in the FGR NESS are given by

$$
\begin{aligned}
& \omega_{\mathcal{S}+}\left(\Phi_{\mathrm{fgr}, j}\right)=2 \pi \sum_{k=1}^{M} \frac{\left|f_{j}\left(\varepsilon_{0}\right)\right|^{2}\left|f_{k}\left(\varepsilon_{0}\right)\right|^{2}}{\left|f\left(\varepsilon_{0}\right)\right|^{2}} \varepsilon_{0}\left(\rho_{j}\left(\varepsilon_{0}\right)-\rho_{k}\left(\varepsilon_{0}\right)\right), \\
& \omega_{\mathcal{S}+}\left(\mathcal{J}_{\mathrm{fgr}, j}\right)=2 \pi \sum_{k=1}^{M} \frac{\left|f_{j}\left(\varepsilon_{0}\right)\right|^{2}\left|f_{k}\left(\varepsilon_{0}\right)\right|^{2}}{\left|f\left(\varepsilon_{0}\right)\right|^{2}}\left(\rho_{j}\left(\varepsilon_{0}\right)-\rho_{k}\left(\varepsilon_{0}\right)\right) .
\end{aligned}
$$

The conservation laws

$$
\sum_{j=1}^{M} \omega_{\mathcal{S}+}\left(\Phi_{\text {fgr }, j}\right)=0, \quad \sum_{j=1}^{M} \omega_{\mathcal{S}+}\left(\mathcal{J}_{\text {fgr }, j}\right)=0,
$$

follow from the definition of the fluxes and the relation $K_{\mathrm{S}}\left(\omega_{\mathcal{S}+}\right)=0$. Of course, they also follow easily from the above explicit formulas. 
Until the end of this subsection we will assume that

$$
\rho_{j}(r)=\frac{1}{1+\mathrm{e}^{\beta_{j}\left(r-\mu_{j}\right)}} .
$$

Using Equ. (8.63), we can also compute the expectation of the entropy production in the FGR NESS $\omega_{\mathcal{S}+}$. The natural extension of the definition (4.25) is

$$
\sigma_{\mathrm{fgr}} \equiv-\sum_{j=1}^{M} \beta_{j}\left(\Phi_{\mathrm{fgr}, j}-\mu_{j} \mathcal{J}_{\mathrm{fgr}, j}\right),
$$

from which we get

$$
\omega_{\mathcal{S}+}\left(\sigma_{\text {fgr }}\right)=2 \pi \sum_{j, k=1}^{M} \frac{\left|f_{j}\left(\varepsilon_{0}\right)\right|^{2}\left|f_{k}\left(\varepsilon_{0}\right)\right|^{2}}{\left|f\left(\varepsilon_{0}\right)\right|^{2}}\left(\rho_{k}\left(\varepsilon_{0}\right)-\rho_{j}\left(\varepsilon_{0}\right)\right) \beta_{j}\left(\varepsilon_{0}-\mu_{j}\right) .
$$

Writing

$$
s_{j} \equiv \log \frac{\rho_{j}\left(\varepsilon_{0}\right)}{1-\rho_{j}\left(\varepsilon_{0}\right)}=\beta_{j}\left(\varepsilon_{0}-\mu_{j}\right),
$$

and symmetrizing the sum in Equ. (8.64) we obtain

$$
\omega_{\mathcal{S}+}\left(\sigma_{\mathrm{fgr}}\right)=\pi \sum_{j, k=1}^{M} \frac{\left|f_{j}\left(\varepsilon_{0}\right)\right|^{2}\left|f_{k}\left(\varepsilon_{0}\right)\right|^{2}}{\left|f\left(\varepsilon_{0}\right)\right|^{2}}\left(\rho_{k}\left(\varepsilon_{0}\right)-\rho_{j}\left(\varepsilon_{0}\right)\right)\left(s_{j}-s_{k}\right),
$$

which is non-negative since $\rho_{l}\left(\varepsilon_{0}\right)$ is a strictly decreasing function of $s_{l}$. The FGR entropy production vanishes iff all $s_{j}$ 's are the same. Note however that this condition does not require that all the $\beta_{j}$ 's and $\mu_{j}$ 's are the same.

Let $\beta_{\mathrm{eq}}$ and $\mu_{\mathrm{eq}}$ be given equilibrium values of the inverse temperature and chemical potential, and

$$
\omega_{\mathcal{S} \text { eq }}=\mathrm{e}^{-\beta_{\mathrm{eq}}\left(H_{\mathcal{S}}-\mu_{\mathrm{eq}}\right)} / \operatorname{Tr}\left(\mathrm{e}^{-\beta_{\mathrm{eq}}\left(H_{\mathcal{S}}-\mu_{\mathrm{eq}}\right)}\right)=\left[\begin{array}{cc}
\left(1+\mathrm{e}^{-\beta_{\mathrm{eq}} \varepsilon_{0}}\right)^{-1} & 0 \\
0 & \left(1+\mathrm{e}^{\beta_{\mathrm{eq}} \varepsilon_{0}}\right)^{-1}
\end{array}\right],
$$

the corresponding NESS. As in Subsection 7.6, the affinities (thermodynamic forces) are $X_{j}=\beta_{\mathrm{eq}}-\beta_{j}$ and $Y_{j}=\beta_{j} \mu_{j}-\beta_{\mathrm{eq}} \mu_{\mathrm{eq}}$. A simple computation yields the FGR Onsager reciprocity relations

$$
\begin{aligned}
&\left.\partial_{X_{j}} \omega_{\mathcal{S}+}\left(\Phi_{\mathrm{fgr}, k}\right)\right|_{X=Y=0}=\left.\partial_{X_{k}} \omega_{\mathcal{S}+}\left(\Phi_{\mathrm{fgr}, j}\right)\right|_{X=Y=0}, \\
&\left.\partial_{Y_{j}} \omega_{\mathcal{S}+}\left(\mathcal{J}_{\mathrm{fgr}, k}\right)\right|_{X=Y=0}=\left.\partial_{Y_{k}} \omega_{\mathcal{S}+}\left(\mathcal{J}_{\mathrm{fgr}, i}\right)\right|_{X=Y=0}, \\
&\left.\partial_{Y_{j}} \omega_{\mathcal{S}+}\left(\Phi_{\mathrm{fgr}, k}\right)\right|_{X=Y=0}=\left.\partial_{X_{k}} \omega_{\mathcal{S}+}\left(\mathcal{J}_{\mathrm{fgr}, i}\right)\right|_{X=Y=0}
\end{aligned}
$$

We set

$$
L_{\mathrm{fgr}}(A, B)=\int_{0}^{\infty} \omega_{\mathcal{S} \text { eq }}\left(\mathrm{e}^{t K_{\mathrm{H}}}(A) B\right) \mathrm{d} t
$$


where $A$ and $B$ are the FGR heat or charge flux observables. Explicit computations yield the FGR Kubo formulas

$$
\begin{aligned}
\left.\partial_{X_{k}} \omega_{\mathcal{S}+}\left(\Phi_{\mathrm{fgr}, j}\right)\right|_{X=Y=0}=L_{\mathrm{fgr}}\left(\Phi_{\mathrm{fgr}, j}, \Phi_{\mathrm{fgr}, k}\right), \\
\left.\partial_{Y_{k}} \omega_{\mathcal{S}+}\left(\Phi_{\mathrm{fgr}, j}\right)\right|_{X=Y=0}=L_{\mathrm{fgr}}\left(\Phi_{\mathrm{fgr}, j}, \mathcal{J}_{\mathrm{fgr}, k}\right), \\
\left.\partial_{X_{k}} \omega_{\mathcal{S}+}\left(\mathcal{J}_{\mathrm{fgr}, j}\right)\right|_{X=Y=0}=L_{\mathrm{fgr}}\left(\mathcal{J}_{\mathrm{fgr}, j}, \Phi_{\mathrm{fgr}, k}\right), \\
\left.\partial_{Y_{k}} \omega_{\mathcal{S}+}\left(\mathcal{J}_{\mathrm{fgr}, j}\right)\right|_{X=Y=0}=L_{\mathrm{fgr}}\left(\mathcal{J}_{\mathrm{fgr}, j}, \mathcal{J}_{\mathrm{fgr}, k}\right) .
\end{aligned}
$$

\subsection{From microscopic to FGR thermodynamics}

At the end of Subsection 4.5 we have briefly discussed the passage from the microscopic to the FGR thermodynamics. We now return to this subject in the context of the SEBB model. The next theorem is a mathematically rigorous version of the heuristic statement that the FGR thermodynamics is the first non-trivial contribution (in $\lambda$ ) to the microscopic thermodynamics.

Theorem 8.2 (i) For any diagonal observable $A \in \mathcal{O}_{\mathcal{S}}$,

$$
\lim _{\lambda \rightarrow 0} \omega_{\lambda+}(A)=\omega_{\mathcal{S}+}(A) .
$$

(ii) For $j=1, \cdots, M$,

$$
\lim _{\lambda \rightarrow 0} \lambda^{-2} \omega_{\lambda+}\left(\Phi_{j}\right)=\omega_{\mathcal{S}+}\left(\Phi_{\mathrm{fgr}, j}\right), \quad \lim _{\lambda \rightarrow 0} \lambda^{-2} \omega_{\lambda+}\left(\mathcal{J}_{j}\right)=\omega_{\mathcal{S}+}\left(\mathcal{J}_{\mathrm{fgr}, j}\right) .
$$

(iii) Let $s_{j} \equiv \log \rho_{j}\left(\varepsilon_{0}\right) /\left(1-\rho_{j}\left(\varepsilon_{0}\right)\right)$ and define the FGR entropy production by

$$
\sigma_{\mathrm{fgr}} \equiv 2 \pi \sum_{j=1}^{M}\left|f_{j}\left(\varepsilon_{0}\right)\right|^{2} s_{j}\left[\begin{array}{c}
-\rho_{j}\left(\varepsilon_{0}\right) \\
1-\rho_{j}\left(\varepsilon_{0}\right)
\end{array}\right] .
$$

Then

$$
\lim _{\lambda \rightarrow 0} \lambda^{-2} \operatorname{Ep}\left(\omega_{\lambda+}\right)=\omega_{\mathcal{S}+}\left(\sigma_{\text {fgr }}\right) .
$$

The proof of this theorem is an integration exercise. We will restrict ourselves to an outline of the proof of Part (i) and several comments. Let $A=N_{\mathcal{S}}=a^{*}(1) a(1)$. Then

$$
\omega_{\lambda+}(A)=\left(W_{-} 1, T_{\mathcal{R}} W_{-} 1\right)=\sum_{j=1}^{M} \lambda^{2} \int_{e_{-}}^{e_{+}} \frac{\left|f_{j}(r)\right|^{2}}{|F(r)|^{2}} \rho_{j}(r) \mathrm{d} r
$$

and

$$
\omega_{\mathcal{S}+}(A)=\sum_{j=1}^{M} \frac{\left|f_{j}\left(\varepsilon_{0}\right)\right|^{2}}{\left|f\left(\varepsilon_{0}\right)\right|^{2}} \rho_{j}\left(\varepsilon_{0}\right) .
$$

Hence, to prove Part (i) we need to show that

$$
\lim _{\lambda \rightarrow 0} \lambda^{2} \int_{e_{-}}^{e_{+}} \frac{\left|f_{j}(r)\right|^{2}}{|F(r)|^{2}} \rho_{j}(r) \mathrm{d} r=\frac{\left|f_{j}\left(\varepsilon_{0}\right)\right|^{2}}{\left|f\left(\varepsilon_{0}\right)\right|^{2}} \rho_{j}\left(\varepsilon_{0}\right) .
$$


By Assumption (SEBB2), $R(r) \equiv \operatorname{Re} G(r-\mathrm{i} o)$ and $\pi|f(r)|^{2}=\operatorname{Im} G(r-\mathrm{i} o)$ are bounded continuous functions. The same is true for $\rho_{j}(r)$ by Assumption (SEBB3). Since

$$
F(r)=\varepsilon_{0}-r-\lambda^{2} R(r)+\mathrm{i} \lambda^{2} \pi|f(r)|^{2},
$$

we have

$$
\int_{e_{-}}^{e_{+}} \frac{\left|f_{j}(r)\right|^{2}}{|F(r)|^{2}} \rho_{j}(r) \mathrm{d} r=\int_{e_{-}}^{e_{+}} \frac{\left|f_{j}(r)\right|^{2} \rho_{j}(r)}{\left(r-\varepsilon_{0}+\lambda^{2} R(r)\right)^{2}+\pi^{2} \lambda^{4}|f(r)|^{4}} \mathrm{~d} r .
$$

Using the above-mentioned continuity and boundedness properties it is not hard to show that

$$
\begin{aligned}
& \lim _{\lambda \rightarrow 0} \lambda^{2} \int_{e_{-}}^{e_{+}} \frac{\left|f_{j}(r)\right|^{2}}{|F(r)|^{2}} \rho_{j}(r) \mathrm{d} r \\
& =\rho_{j}\left(\varepsilon_{0}\right)\left|f_{j}\left(\varepsilon_{0}\right)\right|^{2} \lim _{\lambda \rightarrow 0} \lambda^{2} \int_{e_{-}}^{e_{+}} \frac{\mathrm{d} r}{\left(r-\varepsilon_{0}+\lambda^{2} R(r)\right)^{2}+\pi^{2} \lambda^{4}|f(r)|^{4}} \\
& =\rho_{j}\left(\varepsilon_{0}\right)\left|f_{j}\left(\varepsilon_{0}\right)\right|^{2} \lim _{\lambda \rightarrow 0} \lambda^{2} \int_{-\infty}^{\infty} \frac{\mathrm{d} r}{r^{2}+\pi^{2} \lambda^{4}\left|f\left(\varepsilon_{0}\right)\right|^{4}} \\
& =\frac{\left|f_{j}\left(\varepsilon_{0}\right)\right|^{2}}{\left|f\left(\varepsilon_{0}\right)\right|^{2}} \rho_{j}\left(\varepsilon_{0}\right) .
\end{aligned}
$$

The proofs of Parts (ii) and (iii) are similar. Clearly, under additional regularity assumptions one can get information on the rate of convergence in Parts (i)-(iii). Finally, it is not difficult to show, using the Kubo formulas described in Subsection 7.6 and 8.3, that

$$
\lim _{\lambda \rightarrow 0} \lambda^{-2} L_{\rho}(A, B)=L_{\mathrm{fgr}}\left(A_{\mathrm{fgr}}, B_{\mathrm{fgr}}\right),
$$

where $A, B$ are the microscopic heat or charge flux observables and $A_{\mathrm{fgr}}, B_{\mathrm{fgr}}$ are their FGR counterparts.

\section{Appendix}

\subsection{Structural theorems}

Proof of Theorem 3.3 Recall that $\pi_{\omega}(\mathcal{O})^{\prime \prime}$ is the Banach space dual of $\mathcal{N}_{\omega}$. If $A \in \mathcal{O}$ and $\tilde{A} \in \pi_{\omega}(\mathcal{O})^{\prime \prime}$ is a weak-* accumulation point of the net

$$
\frac{1}{t} \int_{0}^{t} \pi_{\omega}\left(\tau_{V}^{s}(A)\right) \mathrm{d} s
$$

$t \geq 0$, it follows from the asymptotic abelianness in mean that $\tilde{A} \in \pi_{\omega}(\mathcal{O})^{\prime}$. Since $\omega$ is a factor state we have $\pi_{\omega}(\mathcal{O})^{\prime} \cap \pi_{\omega}(\mathcal{O})^{\prime \prime}=\mathbb{C} I$ and therefore, for any $\eta \in \mathcal{N}_{\omega}$, one has

$$
\eta(\tilde{A})=\omega(\tilde{A}) \text {. }
$$


Let $\mu, \nu \in \mathcal{N}_{\omega}$ and $\mu_{+} \in \Sigma_{+}\left(\mu, \tau_{V}\right)$. Let $t_{\alpha} \rightarrow \infty$ be a net such that

$$
\lim _{\alpha} \frac{1}{t_{\alpha}} \int_{0}^{t_{\alpha}} \mu \circ \tau_{V}^{s}(A) \mathrm{d} s=\mu_{+}(A)
$$

for all $A \in \mathcal{O}$. Passing to a subnet, we may also assume that for all $A \in \mathcal{O}$ and some $\nu_{+} \in \Sigma_{+}\left(\nu, \tau_{V}\right)$,

$$
\lim _{\alpha} \frac{1}{t_{\alpha}} \int_{0}^{t_{\alpha}} \nu \circ \tau_{V}^{s}(A) \mathrm{d} s=\nu_{+}(A) .
$$

By the Banach-Alaoglu theorem, for any $A \in \mathcal{O}$ there exists a subnet $t_{\gamma}(A)$ of the net $t_{\alpha}$ and $A^{\#} \in \pi_{\omega}(\mathcal{O})^{\prime \prime}$ such that, for all $\eta \in \mathcal{N}_{\omega}$

$$
\lim _{\gamma} \frac{1}{t_{\gamma}(A)} \int_{0}^{t_{\gamma}(A)} \eta\left(\pi_{\omega}\left(\tau_{V}^{s}(A)\right)\right) \mathrm{d} s=\eta\left(A^{\#}\right) .
$$

Hence, $\mu_{+}(A)=\mu\left(A^{\#}\right)$ and $\nu_{+}(A)=\nu\left(A^{\#}\right)$. By (9.67) we also have $\mu\left(A^{\#}\right)=$ $\omega\left(A^{\#}\right)=\nu\left(A^{\#}\right)$ and so $\mu_{+}(A)=\nu_{+}(A)$. We conclude that $\mu_{+}=\nu_{+}$and that

$$
\Sigma_{+}\left(\mu, \tau_{V}\right) \subset \Sigma_{+}\left(\nu, \tau_{V}\right) .
$$

By symmetry, the reverse inclusion also holds and

$$
\Sigma_{+}\left(\mu, \tau_{V}\right)=\Sigma_{+}\left(\omega, \tau_{V}\right)
$$

for all $\mu \in \mathcal{N}_{\omega}$

Proof of Theorem 3.6 To prove this theorem we use the correspondence between $\omega$-normal states and elements of the standard cone $\mathcal{P}$ obtained from $\omega$ (see Proposition 37 in [Pi]); this is possible since $\omega$ is modular by assumption.

Note that if $\operatorname{Ker} L_{V} \neq\{0\}$, then there is an $\omega$-normal, $\tau_{V}$-invariant state $\eta$. By Theorem 3.3, $\Sigma_{+}\left(\omega, \tau_{V}\right)=\Sigma_{+}\left(\eta, \tau_{V}\right)$ and obviously $\Sigma_{+}\left(\eta, \tau_{V}\right)=\{\eta\}$. Two nonzero elements in $\operatorname{Ker} L_{V}$ therefore yield the same vector state and are represented by the same vector in the standard cone, i.e., $\operatorname{Ker} L_{V} \cap \mathcal{P}$ is a one-dimensional half-line. Recall that any $\zeta \in \mathfrak{h}_{\omega}$ can be uniquely decomposed as

$$
\zeta=\zeta_{1}-\zeta_{2}+\mathrm{i} \zeta_{3}-\mathrm{i} \zeta_{4}
$$

with $\zeta_{i}$ in $\mathcal{P}$. Since $e^{\mathrm{i} t L_{V}}$ preserves the standard cone, $e^{\mathrm{i} t L_{V}} \zeta=\zeta$ iff $e^{\mathrm{i} t L_{V}} \zeta_{i}=\zeta_{i}$ for all $i$ (i.e., $\zeta_{i} \in \operatorname{Ker} L_{V} \cap \mathcal{P}$ for all $i$ ). Hence, $\operatorname{Ker} L_{V}$ is one-dimensional and Part (i) follows.

The proof of Part (ii) is simple. Any NESS $\eta \in \Sigma_{+}\left(\omega, \tau_{V}\right)$ can be uniquely decomposed as $\eta_{n}+\eta_{s}$ where $\eta_{n} \ll \omega$ and $\eta_{s} \perp \omega$. Since $\eta$ is $\tau_{V}$-invariant, $\eta_{n}$ and $\eta_{s}$ are also $\tau_{V}$-invariant. Therefore $\eta_{n}$ is represented by a vector $\zeta$ in $\operatorname{Ker} L_{V} \cap \mathcal{P}$. If $\operatorname{Ker} L_{V}=\{0\}$, then $\eta_{n}=0$ and $\eta \perp \omega$. 
It remains to prove Part (iii) (see Theorem 44 in the lecture notes [Pi]). Let $\varphi \in$ Ker $L_{V}$ be a separating vector for $\mathfrak{M}_{\omega}$. Let $B \in \pi_{\omega}(\mathcal{O})^{\prime}$ be such that $\|B \varphi\|=1$ and let $\nu_{B}$ be the vector state associated to $B \varphi, \nu_{B}(\cdot)=(B \varphi, \cdot B \varphi)$. For any $A \in \pi_{\omega}(\mathcal{O})$,

$$
\begin{aligned}
\frac{1}{t} \int_{0}^{t} \nu_{B}\left(\tau_{V}^{s}(A)\right) \mathrm{d} s & =\frac{1}{t} \int_{0}^{t}\left(B \varphi, e^{\mathrm{i} s L_{V}} \pi_{\omega}(A) e^{-\mathrm{i} s L_{V}} B \varphi\right) \mathrm{d} s \\
& =\left(\frac{1}{t} \int_{0}^{t} e^{-\mathrm{i} s L_{V}} B^{*} B \varphi \mathrm{d} s, \pi_{\omega}(A) \varphi\right) .
\end{aligned}
$$

Hence, by the von Neumann ergodic theorem,

$$
\nu_{B+}(A) \equiv \lim _{t \rightarrow \infty} \frac{1}{t} \int_{0}^{t} \nu_{B}\left(\tau_{V}^{s}(A)\right) \mathrm{d} s=\left(P_{\operatorname{Ker} L_{V}} B^{*} B \varphi, \pi_{\omega}(A) \varphi\right),
$$

where $P_{\operatorname{Ker} L_{V}}$ is the projection on $\operatorname{Ker} L_{V}$. Since $\varphi$ is cyclic for $\pi_{\omega}(\mathcal{O})^{\prime}$, for every $n \in \mathbb{N}$ we can find a $B_{n}$ such that $\left\|\omega-\nu_{B_{n}}\right\|<1 / n$. The sequence $\nu_{B_{n}}$ is Cauchy in norm and for all $\omega_{+} \in \Sigma_{+}\left(\omega, \tau_{V}\right)$,

$$
\left\|\omega_{+}-\nu_{B_{n}+}\right\| \leq\left\|\omega-\nu_{B_{n}}\right\|<1 / n
$$

This implies that the norm limit of $\nu_{B_{n}}$ is the unique NESS in $\Sigma_{+}\left(\omega, \tau_{V}\right)$. Since $\nu_{B_{n}}+\in \mathcal{N}_{\omega}$ and $\mathcal{N}_{\omega}$ is a norm closed subset of $\mathcal{O}^{*}$, this NESS is $\omega$-normal.

\subsection{The Hilbert-Schmidt condition}

Proof of Theorem 7.2 We will prove that $T_{+}^{1 / 2}-T^{1 / 2}$ is Hilbert-Schmidt. The proof that $\left(I-T_{+}\right)^{1 / 2}-(I-T)^{1 / 2}$ is also Hilbert-Schmidt is identical. For an elementary introduction to Hilbert-Schmidt operators (which suffices for the proof below) the reader may consult Section VI.6 in [RS].

By our general assumptions, the functions $f(r)$ and $F(r)^{-1}$ are bounded and continuous. By the assumption of Theorem 7.2, all the densities $\rho_{j}(r)$ are the same and equal to $\rho(r)$. Hence,

$$
T_{\mathcal{R}}=\bigoplus_{j=1}^{M} \rho_{j}(r)=\rho\left(h_{\mathcal{R}}\right)
$$

Let $p_{\mathcal{R}}$ be the orthogonal projection on the reservoir Hilbert space $\mathfrak{h}_{\mathcal{R}}$. Since $T^{1 / 2}-$ $T_{\mathcal{R}}^{1 / 2}=T_{\mathcal{S}}^{1 / 2}, T_{+}^{1 / 2}\left(I-p_{\mathcal{R}}\right),\left(I-p_{\mathcal{R}}\right) T_{+}^{1 / 2}$ are obviously Hilbert-Schmidt, it suffices to show that $p_{\mathcal{R}} T_{+}^{1 / 2} p_{\mathcal{R}}-T_{\mathcal{R}}^{1 / 2}$ is a Hilbert-Schmidt operator on the Hilbert space $\mathfrak{h}_{\mathcal{R}}$. Since

$$
p_{\mathcal{R}} T_{+}^{1 / 2} p_{\mathcal{R}}-T_{\mathcal{R}}^{1 / 2}=-p_{\mathcal{R}} W_{-}^{*}\left[W_{-} p_{\mathcal{R}}, T_{\mathcal{R}}^{1 / 2}\right]
$$

it suffices to show that $K \equiv\left[W_{-} p_{\mathcal{R}}, T_{\mathcal{R}}^{1 / 2}\right]$ is a Hilbert-Schmidt operator on $\mathfrak{h}_{\mathcal{R}}$. By Theorem 6.2, for $g \in \mathfrak{h}_{\mathcal{R}}$,

$$
(K g)(r)=\lambda^{2} \frac{f(r)}{F(r)} \int_{e_{-}}^{e_{+}} \frac{\rho\left(r^{\prime}\right)^{1 / 2}-\rho(r)^{1 / 2}}{r^{\prime}-r+\mathrm{i} o} \bar{f}\left(r^{\prime}\right) \cdot g\left(r^{\prime}\right) \mathrm{d} r^{\prime} .
$$


Let $K_{i j}$ be an operator on $L^{2}\left(\left(e_{-}, e_{+}\right), \mathrm{d} r\right)$ defined by

$$
\left(K_{i j} h\right)(r)=\lambda^{2} \frac{f_{i}(r)}{F(r)} \int_{e_{-}}^{e_{+}} \frac{\rho\left(r^{\prime}\right)^{1 / 2}-\rho(r)^{1 / 2}}{r^{\prime}-r+\mathrm{i} o} \bar{f}_{j}\left(r^{\prime}\right) h\left(r^{\prime}\right) \mathrm{d} r^{\prime} .
$$

To prove that $K$ is Hilbert-Schmidt on $\mathfrak{h}_{\mathcal{R}}$, it suffices to show that $K_{i j}$ is HilbertSchmidt on $L^{2}\left(\left(e_{-}, e_{+}\right), \mathrm{d} r\right)$ for all $i, j$.

Let $h_{1}, h_{2} \in L^{2}\left(\left(e_{-}, e_{+}\right), \mathrm{d} r\right)$ be bounded continuous functions. Then

$$
\left(h_{1}, K_{i j} h_{2}\right)=\lambda^{2} \int_{e_{-}}^{e_{+}} \frac{\bar{h}_{1}(r) f_{i}(r) g_{2}(r)}{F(r)} \mathrm{d} r
$$

where

$$
g_{2}(r)=\lim _{\epsilon \downarrow 0} \int_{e_{-}}^{e_{+}} \frac{\rho\left(r^{\prime}\right)^{1 / 2}-\rho(r)^{1 / 2}}{r^{\prime}-r+\mathrm{i} \epsilon} \bar{f}_{j}\left(r^{\prime}\right) h_{2}\left(r^{\prime}\right) \mathrm{d} r^{\prime} .
$$

Using the identity

$$
\frac{1}{r^{\prime}-r+\mathrm{i} \epsilon}=\frac{r^{\prime}-r}{\left(r^{\prime}-r\right)^{2}+\epsilon^{2}}-\frac{\mathrm{i} \epsilon}{\left(r^{\prime}-r\right)^{2}+\epsilon^{2}},
$$

and the fact that, for $r \in\left(e_{-}, e_{+}\right)$, one has

$$
\begin{aligned}
\lim _{\epsilon \downarrow 0} \epsilon \int_{e_{-}}^{e_{+}} \frac{\rho\left(r^{\prime}\right)^{1 / 2}-\rho(r)^{1 / 2}}{\left(r^{\prime}-r\right)^{2}+\epsilon^{2}} \bar{f}_{j}\left(r^{\prime}\right) h_{2}\left(r^{\prime}\right) \mathrm{d} r^{\prime} & =\pi\left(\rho(r)^{1 / 2}-\rho(r)^{1 / 2}\right) \bar{f}_{j}(r) h_{2}(r) \\
& =0,
\end{aligned}
$$

(see the Lecture $[\mathrm{Ja}]$ ), we obtain

$$
g_{2}(r)=\lim _{\epsilon \downarrow 0} \int_{e_{-}}^{e_{+}} \frac{\left(r^{\prime}-r\right)\left(\rho\left(r^{\prime}\right)^{1 / 2}-\rho(r)^{1 / 2}\right)}{\left(r^{\prime}-r\right)^{2}+\epsilon^{2}} \bar{f}_{j}\left(r^{\prime}\right) h_{2}\left(r^{\prime}\right) \mathrm{d} r^{\prime} .
$$

Since $f_{j}$ and $h_{2}$ are bounded and $\rho(r)^{1 / 2}$ is $\frac{1}{2}$-Hölder continuous, we have

$$
\begin{gathered}
\sup _{\epsilon>0, r \in\left(e_{-}, e_{+}\right)}\left|\int_{e_{-}}^{e_{+}} \frac{\left(r^{\prime}-r\right)\left(\rho\left(r^{\prime}\right)^{1 / 2}-\rho(r)^{1 / 2}\right)}{\left(r^{\prime}-r\right)^{2}+\epsilon^{2}} \bar{f}_{j}\left(r^{\prime}\right) h_{2}\left(r^{\prime}\right) \mathrm{d} r^{\prime}\right| \\
\leq C \sup _{r \in\left(e_{-}, e_{+}\right)} \int_{e_{-}}^{e_{+}} \frac{\bar{f}_{j}\left(r^{\prime}\right) h_{2}\left(r^{\prime}\right)}{\left|r^{\prime}-r\right|^{1 / 2}} \mathrm{~d} r^{\prime}<\infty .
\end{gathered}
$$

Moreover, since $\bar{h}_{1}(r) F(r)^{-1} f_{i}(r) \in L^{1}\left(\left(e_{-}, e_{+}\right), \mathrm{d} r\right)$, we can invoke the dominated convergence theorem to rewrite Equ. (9.68) as

$$
\left(h_{1}, K_{i j} h_{2}\right)=\lim _{\epsilon \downarrow 0}\left(h_{1}, K_{i j, \epsilon} h_{2}\right)
$$

where $K_{i j, \epsilon}$ is the integral operator on $L^{2}\left(\left(e_{-}, e_{+}\right), \mathrm{d} r\right)$ with kernel

$$
k_{\epsilon}\left(r, r^{\prime}\right)=\lambda^{2} \frac{f_{i}(r) \bar{f}_{j}\left(r^{\prime}\right)}{F(r)} \frac{\left(r^{\prime}-r\right)\left(\rho\left(r^{\prime}\right)^{1 / 2}-\rho(r)^{1 / 2}\right)}{\left(r^{\prime}-r\right)^{2}+\epsilon^{2}} .
$$


We denote by $\|\cdot\|_{\text {HS }}$ the Hilbert-Schmidt norm. Then

$$
\left\|K_{i j, \epsilon}\right\|_{\mathrm{HS}}^{2}=\int\left|k_{\epsilon}\left(r, r^{\prime}\right)\right|^{2} \mathrm{~d} r \mathrm{~d} r^{\prime} .
$$

Since $\rho(r)^{1 / 2}$ is $\alpha$-Hölder continuous for $\alpha>1 / 2$ and $F(r)^{-1}$ is bounded there exists a constant $C$ such that, for $r, r^{\prime} \in\left(e_{-}, e_{+}\right)$and $\epsilon>0$, one has the estimate

$$
\left|k_{\epsilon}\left(r, r^{\prime}\right)\right|^{2} \leq C \frac{\left|f_{i}(r)\right|^{2}\left|f_{j}\left(r^{\prime}\right)\right|^{2}}{\left|r-r^{\prime}\right|^{2(1-\alpha)}} .
$$

Therefore, since $2(1-\alpha)<1$, we conclude that

$$
\sup _{\epsilon>0}\left\|K_{i j, \epsilon}\right\|_{\mathrm{HS}}^{2}=\sup _{\epsilon>0} \int\left|k_{\epsilon}\left(r, r^{\prime}\right)\right|^{2} \mathrm{~d} r \mathrm{~d} r^{\prime}<\infty .
$$

The Hilbert-Schmidt class of operators on $L^{2}\left(\left(e_{-}, e_{+}\right), \mathrm{d} r\right)$ is a Hilbert space with the inner product $(X, Y)=\operatorname{Tr}\left(X^{*} Y\right)$. Since $\left\{K_{i j, \epsilon}\right\}_{\epsilon>0}$ is a bounded set in this Hilbert space, there is a sequence $\epsilon_{n} \rightarrow 0$ and a Hilbert-Schmidt operator $\tilde{K}_{i j}$ such that for any Hilbert-Schmidt operator $X$ on $L^{2}\left(\left(e_{-}, e_{+}\right), \mathrm{d} r\right)$,

$$
\lim _{n \rightarrow \infty} \operatorname{Tr}\left(X^{*} K_{i j, \epsilon_{n}}\right)=\operatorname{Tr}\left(X^{*} \tilde{K}_{i j}\right)
$$

Taking $X=\left(h_{1}, \cdot\right) h_{2}$, where $h_{i} \in L^{2}\left(\left(e_{-}, e_{+}\right), \mathrm{d} r\right)$ are bounded and continuous, we derive from (9.69) that $\left(h_{1}, \tilde{K}_{i j} h_{2}\right)=\left(h_{1}, K_{i j} h_{2}\right)$. Since the set of such $h$ 's is dense in $L^{2}\left(\left(e_{-}, e_{+}\right), \mathrm{d} r\right), \tilde{K}_{i j}=K_{i j}$ and so $K_{i j}$ is Hilbert-Schmidt. 


\section{References}

[AB] Araki, H., Barouch, E.: On the dynamics and ergodic properties of the $X Y$ model. J. Stat. Phys. 31, 327 (1983).

[AJPP] Aschbacher, W., Jakšić, V., Pautrat, Y., Pillet, C.-A.: Transport properties of ideal Fermi gases (in preparation).

[AM] Aizenstadt, V.V., Malyshev, V.A.: Spin interaction with an ideal Fermi gas. J. Stat. Phys. 48, 51 (1987).

[Ar1] Araki, H.: Relative entropy of states of von Neumann algebras. Publ. Res. Inst. Math. Sci. Kyoto Univ. 11, 809 (1975/76).

[Ar2] Araki, H.: Relative entropy of states of von Neumann algebras II. Publ. Res. Inst. Math. Sci. Kyoto Univ. 13, 173 (1977/78).

[Ar3] Araki, H.: On the $X Y$-model on two-sided infinite chain. Publ. Res. Inst. Math. Sci. Kyoto Univ. 20, 277 (1984).

[ArM] Araki, H., Masuda, T.: Positive cones and $L^{p}$-spaces for von Neumann algebras. Publ. RIMS Kyoto Univ. 18, 339 (1982).

[At] Attal, S.: Elements of operator algebras and modular theory. Volume I of this series.

[AW] Araki, H., Wyss, W.: Representations of canonical anti-commutation relations. Helv. Phys. Acta 37, 136 (1964).

[BFS] Bach, V., Fröhlich, J., Sigal, I.: Return to equilibrium. J. Math. Phys. 41, 3985 (2000).

[BLR] Bonetto, F., Lebowitz, J.L., Rey-Bellet, L.: Fourier Law: A challenge to theorists. In Mathematical Physics 2000. Imp. Coll. Press, London (2000).

[BM] Botvich, D.D., Malyshev, V.A.: Unitary equivalence of temperature dynamics for ideal and locally perturbed Fermi Gas. Commun. Math. Phys. 61, 209 (1978).

[BR1] Bratteli, O, Robinson D. W.: Operator Algebras and Quantum Statistical Mechanics 1. Springer, Berlin (1987).

[BR2] Bratteli, O, Robinson D. W.: Operator Algebras and Quantum Statistical Mechanics 2. Springer, Berlin (1996).

[BSZ] Baez, J.C., Segal, I.E., Zhou, Z.: Introduction to algebraic and constructive quantum field theory. Princeton University Press, Princeton NJ, (1991).

[CG1] Cohen, E.G.D., Gallavotti, G.: Dynamical ensembles in stationary states. J. Stat. Phys. 80, 931 (1995). 
[CG2] Cohen, E.G.D., Gallavotti, G.: Dynamical ensembles in nonequilibrium statistical mechanics. Phys. Rev. Lett. 74, 2694 (1995).

[Da1] Davies, E.B.: Markovian master equations. Commun. Math. Phys. 39, 91 (1974).

[Da2] Davies, E.B.: Markovian master equations II. Math. Ann. 219, 147 (1976).

[De] Dell'Antonio, G.F.: Structure of the algebra of some free systems. Commun. Math. Phys. 9, 81 (1968).

[DGM] De Groot, S.R., Mazur, P.: Non-Equilibrium Thermodynamics. NorthHolland, Amsterdam (1969).

[D1] Dereziński, J.: Fermi Golden Rule and open quantum systems. This volume.

[D2] Dereziński, J.: Inroduction to representations of canonical commutation and anticommutation relations. Lecture notes of the Nordfjordeid Summer School "Large Coulomb Systems-Quantum Electrodynamics", August 2003.

[Di] Dirac P.A.M.: The quantum theory of the emission and absorption of radiation. Proc. Roy. Soc. London, Ser. A 114, 243 (1927).

[DJ] Dereziński, J., Jakšić, V.: Return to equilibrium for Pauli-Fierz systems. Ann. Henri Poincaré 4, 739 (2003).

[DJP] Dereziński, J., Jakšić, V., Pillet, C.-A.: Perturbation theory of $W^{*}$-dynamics, KMS-states and Liouvillean. Rev. Math. Phys. 15, 447 (2003).

[Do] Dorfman, J.R.: An Introduction to Chaos in Nonequilibrium Statistical Mechanics. Cambridge University Press, Cambridge (1999)

[Ei] Einstein, A.: Zur Quantentheorie der Strahlung. Physik. Zeitschr. 18, 121 (1917). This paper is reprinted in: van der Waerden, B.L., Sources of Quantum Mechanics. Dover, New York (1967).

[EM] Evans, D.J., Morriss, G.P.: Statistical Mechanics of Non-Equilibrium Liquids. Academic Press, New York (1990).

[Fer] Fermi, E.: Nuclear Physics. Notes compiled by Orear J., Rosenfeld A.H. and Schluter R.A. The University of Chicago Press, Chicago, 1950.

[FM1] Fröhlich, J., Merkli, M.: Thermal Ionization. Mathematical Physics, Analysis and Geometry 7, 239 (2004).

[FM2] Fröhlich, J., Merkli, M.: Another return of "return to equilibrium". Commun. Math. Phys., 251, 235 (2004).

[FMS] Fröhlich, J., Merkli, M., Sigal, I.M.: Ionization of atoms in a thermal field. J. Stat. Phys. 116, 311 (2004). 
[FMSU] Fröhlich, J., Merkli, M., Schwarz, S., Ueltschi, D.: Statistical mechanics of thermodynamic processes. In A Garden of Quanta, 345. World Scientific Publishing, River Edge NJ (2003).

[FMU] Fröhlich, J., Merkli, M., Ueltschi, D.: Dissipative transport: thermal contacts and tunneling junctions. Ann. Henri Poincaré 4, 897 (2004).

[Fr] Friedrichs, K. O.: Perturbation of Spectra in Hilbert Space, AMS, Providence (1965).

[Ga1] Gallavotti, G.: Nonequilibrium thermodynamics. Preprint, mp-arc 03-11 (2003).

[Ga2] Gallavotti, G.: Entropy production in nonequilibrium thermodynamics: a review. Preprint, arXiv cond-mat/0312657 (2003).

[GVV1] Goderis, D., Verbeure, A., Vets, P.: Noncommutative central limits. Probab. Theory Related Fields 82527 (1989).

[GVV2] Goderis, V., Verbeure, A., Vets, P.: Quantum central limit and coarse graining. In Quantum probability and applications, $V$. Lecture Notes in Math., 1442, 178 (1988).

[GVV3] Goderis, D., Verbeure, A., Vets, P.: About the mathematical theory of quantum fluctuations. In Mathematical Methods in Statistical Mechanics. Leuven Notes Math. Theoret. Phys. Ser. A Math. Phys., 1, 31. Leuven Univ. Press, Leuven (1989).

[GVV4] Goderis, D., Verbeure, A., Vets, P.: Theory of quantum fluctuations and the Onsager relations. J. Stat. Phys. 56, 721 (1989).

[GVV5] Goderis, D., Verbeure, A., Vets, P.: Dynamics of fluctuations for quantum lattice systems. Commun. Math. Phys. 128, 533 (1990).

[GVV6] Goderis, D., Verbeure, A., Vets, P.: About the exactness of the linear response theory. Commun. Math. Phys. 136, 265 (1991).

[Haa] Haake, F.: Statistical Treatment of Open Systems by Generalized Master Equation. Springer Tracts in Modern Physics 66, Springer, Berlin (1973).

[Ha] Haag, R.: Local Quantum Physics. Springer, New York (1993).

[Ja] Jakšić, V.: Topics in spectral theory. Volume I of this series.

[JKP] Jakšić, V., Kritchevski, E., Pillet, C.-A.: Mathematical theory of the WignerWeisskopf atom. Lecture notes of the Nordfjordeid Summer School "Large Coulomb Systems-Quantum Electrodynamics", August 2003.

[Jo] Joye, A.: Introduction to quantum statistical mechanics. Volume I of this series. 
[JP1] Jakšić, V., Pillet, C.-A.: On a model for quantum friction II: Fermi's golden rule and dynamics at positive temperature. Commun. Math. Phys. 176, 619 (1996).

[JP2] Jakšić, V., Pillet, C.-A.: On a model for quantum friction III: Ergodic properties of the spin-boson system. Commun. Math. Phys. 178, 627 (1996).

[JP3] Jakšić, V., Pillet, C.-A.: Spectral theory of thermal relaxation. J. Math. Phys. 38, 1757 (1997).

[JP4] Jakšić, V., Pillet, C.-A.: Mathematical theory of non-equilibrium quantum statistical mechanics. J. Stat. Phys. 108, 787 (2002).

[JP5] Jakšić, V., Pillet, C.-A.: Non-equilibrium steady states for finite quantum systems coupled to thermal reservoirs. Commun. Math. Phys. 226, 131 (2002).

[JP6] Jakšić, V., Pillet, C-A.: On entropy production in quantum statistical mechanics. Commun. Math. Phys. 217, 285 (2001).

[JP7] Jakšić, V., Pillet, C.-A.: A note on the entropy production formula. Contemp. Math. 327, 175 (2003).

[JPR1] Jakšić, V., Pillet, C.-A., Rey-Bellet, L.: Fluctuation of entropy production in classical statistical mechanics. In preparation.

[JPR2] Jakšić, V., Pillet, C.-A., Rey-Bellet, L.: In preparation.

[KR] Kadison R.V., Ringrose J.R.: Fundamentals of the Theory of Operator Algebras II: Advanced Theory, Graduate Studies in Mathematics 16, AMS, Providence (1997).

[LeSp] Lebowitz, J., Spohn, H.: Irreversible thermodynamics for quantum systems weakly coupled to thermal reservoirs. Adv. Chem. Phys. 39, 109 (1978).

[Li] Lindblad, G.: Completely positive maps and entropy inequalities. Commun. Math. Phys. 40, 147 (1975).

[LMS] Lieb, E.H., Schulz, T., Mathis, D.: Two soluble models of an antiferromagnetic chain. Ann. Phys. 28, 407, (1961).

[Ma] Matsui, T.: On the algebra of fluctuation in quantum spin chains. Ann. Henri Poincaré 4, 63 (2003).

[Me1] Merkli, M.: Positive commutators in non-equilibrium quantum statistical mechanics. Commun. Math. Phys. 223, 327 (2001).

[Me2] Merkli, M.: Stability of equilibria with a condensate. Commun. Math. Phys., in press.

[Me3] Merkli, M.: The ideal quantum gas. Volume I of this series.

[Mes] Messiah, A.: Quantum Mechanics. Volume II. Wiley, New York. 
[Og] Ogata, Y:: The stability of the non-equilibrium steady states. Commun. Math. Phys. 245, 577 (2004).

[OP] Ohya, M., Petz, D.: Quantum Entropy and its Use. Springer-Verlag, Berlin (1993).

[Pa] Pais, A.: "Subtle is the Lord...", The Science and Life of Albert Einstein. Oxford University Press, Oxford (1982).

[Pi] Pillet, C.-A.: Quantum dynamical systems and their KMS-states. Volume I of this series.

[PoSt] Powers, R. T., Stormer, E.: Free states of the canonical anticommutation relations. Commun. Math. Phys. 16, 1 (1969).

[RC] Rondoni, L., Cohen, E.G.D.: Gibbs entropy and irreversible thermodynamics. Nonlinearity 13, 1905 (2000).

[Re] Rey-Bellet, L.: Open classical systems. Volume II of this series.

[Ri] Rideau, G.: On some representations of the anticommutation relations. Commun. Math. Phys. 9, 229 (1968).

[Ro1] Robinson, D.W.: Return to equilibrium. Commun. Math. Phys. 31, 171 (1973).

[Ro2] Robinson, D.W.: $C^{*}$-algebras in quantum statistical mechanics. In $C^{*}$ algebras and their Applications to Statistical Mechanics and Quantum Field Theory, (D. Kastler editor). North-Holand, Amsterdam (1976).

[Ru1] Ruelle, D.: Natural nonequilibrium states in quantum statistical mechanics. J. Stat. Phys. 98, 57 (2000).

[Ru2] Ruelle, D.: Entropy production in quantum spin systems. Commun. Math. Phys. 224, 3 (2001).

[Ru3] Ruelle, D.: Topics in quantum statistical mechanics and operator algebras. Preprint, mp-arc 01-257 (2001).

[Ru4] Ruelle, D.: Smooth dynamics and new theoretical ideas in nonequilibrium statistical mechanics. J. Stat. Phys. 95, 393 (1999).

[Ru5] Ruelle, D.: Extending the definition of entropy to nonequilibrium steady states. Proc. Nat. Acad. Sci. USA 100, 3054 (2003).

[Ru6] Ruelle, D.: A remark on the equivalence of isokinetic and isoenergetic thermostats in the thermodynamic limit. J. Stat. Phys. 100, 757 (2000).

[Ru7] Ruelle, D.: Conversations on nonequilibrium physics with an extraterrestrial. Physics Today 57, 48 (2004). 
[RS] Reed, M., Simon, B.: Methods of Modern Mathematical Physics, I. Functional Analysis, London, Academic Press (1980).

[Sp] Spohn, H.: An algebraic condition for the approach to equilibrium of an open $N$-level system, Lett. Math. Phys. 2, 33 (1977).

[Ta] Takesaki, M.: Theory of Operator Algebras I. Springer, New-York (1979).

[TM] Tasaki, S., Matsui, T.: Fluctuation theorem, nonequilibrium steady states and MacLennan-Zubarev ensembles of a class of large quantum systems. Fundamental Aspects of Quantum Physics (Tokyo, 2001). QP-PQ: Quantum Probab. White Noise Anal., 17, 100. World Sci., River Edge NJ, (2003).

[VH1] van Hove, L.: Quantum-mechanical perturbations giving rise to a statistical transport equation. Physica 21, 517.

[VH2] van Hove, L.: The approach to equilibrium in quantum statistics. Physica 23, 441.

[VH3] van Hove, L.: Master equation and approach to equilibrium for quantum systems. In Fundamental problems in statistical mechanics, compiled by E.G.D. Cohen, North-Holand, Amsterdam 1962.

[WW] Weisskopf, V., Wigner, E.: Berechnung der natürlichen Linienbreite auf Grund der Diracschen Lichttheorie. Zeitschrift für Physik 63, 54 (1930). 